RECIPROCAL RISK: THE LONGITUDINAL RELATIONSHIP BETWEEN

EMOTION REGULATION AND NON-SUICIDAL SELF-INJURY

BY

KEALAGH ROBINSON

\begin{abstract}
A thesis
Submitted to the Victoria University of Wellington in

fulfilment of the requirements for the degree of

Master of Science
\end{abstract}

Victoria University of Wellington

2017 


\begin{abstract}
Poor emotion regulation has been highlighted as a potential risk factor for the development and maintenance of Non-Suicidal Self-Injury (NSSI; Fox et al., 2015). However, longitudinal research tracking the relationship between emotion regulation and NSSI during adolescence is limited. In addition, the conceptual argument that NSSI may in turn be a risk factor for poor emotion regulation (Gratz, 2003), remains largely untested. Three studies, all drawn from the Youth Wellbeing Study, were conducted to investigate the developmental relationship between emotion regulation and NSSI during adolescence. Study One established the psychometric properties of the Emotion Regulation Index for Children and Adolescents in a large sample of adolescents and validated the self-report measure for use in Studies Two and Three. Study Two assessed the longitudinal relationship between emotion regulation and NSSI within a cohort of young adolescents across a three year period. Consistent with previous research, poor emotion regulation predicted subsequent engagement in NSSI for both boys and girls, suggesting that poor emotion regulation may be causally implicated in the development of NSSI behaviours. Critically, this relationship was reciprocal; engaging in NSSI also predicted poorer subsequent emotion regulation. Study Three tests the hypothesis that NSSI 'damages' emotion regulation by impairing the interpersonal relationships which underlie the development of emotion regulation skills. Using a quasi-longitudinal multiple mediation analysis, the combination of Time Two Parental Attachment and Time Two Peer Attachment were found to fully mediate the relationship between Time One NSSI and Time Three Emotion Regulation, emphasising the primacy of these relationships during adolescence. This research is the first to empirically demonstrate the reciprocal complexity of the relationship between emotion regulation and NSSI, suggesting that the dynamic relationship between these two factors underlies the development of NSSI during adolescence.
\end{abstract}




\section{Acknowledgements}

This thesis would not have been possible without the unfailing support and guidance of my supervisors Professor Marc Wilson and Dr Gina Grimshaw and the mentorship of Dr Jessica Garisch. I am profoundly grateful for the belief you have in me, and for consistently going above and beyond in assisting me throughout this process. Thank you for teaching me both the privileges and responsibilities of being a psychological scientist.

I also owe a huge debt of thanks to the Youth Wellbeing Study team (past and present) for the mammoth energy and passion that goes into the project, particularly the longitudinal and cross-sectional surveys this thesis draws from. Thanks especially to Maddy Brockelsby, Dr Jessica Garisch, Angelique O’Connell, Professor Marc Wilson, Tahlia Kingi, and Gloria Fraser. I am also grateful for the friendship and encouragement of the Cognitive and Affective Neuroscience Lab, particularly Hazel Godfrey, Sophie Hedley, Amy Maddock, Laura Kranz, Angus Chapman, Dr Christel Devue and Dr Michael Tooley. This thesis would not have been nearly as enjoyable or rewarding without your companionship and support.

In this thesis, as in everything, my family are the cornerstone. Thanks to Mum for always believing the best in me, Dad for the pep talks, and Fergus for the coffee breaks. Pascarn and Eleanor, thank you for keeping me sane during the year and for all your help during the writing process. But mostly thank you for the snacks and animal videos.

This thesis was made possible due to research funding from the Health Research Council of New Zealand, as well as financial support from Victoria University of Wellington Master's (by thesis) Fees Scholarship, an Izard Scholarship and a Graduate Women Wellington Masters by thesis Scholarship. I am very grateful for this financial support. 
Table of Contents

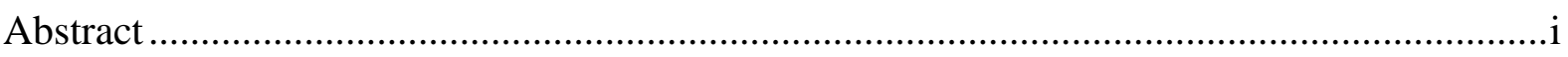

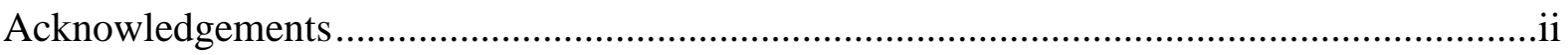

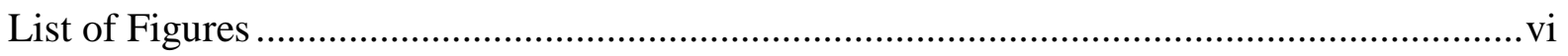

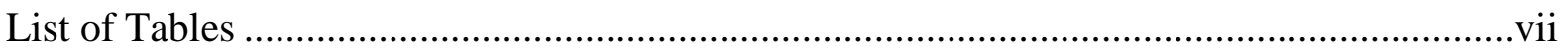

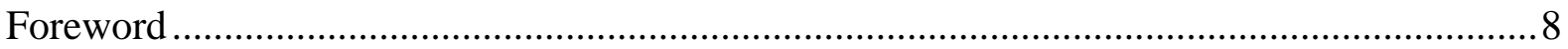

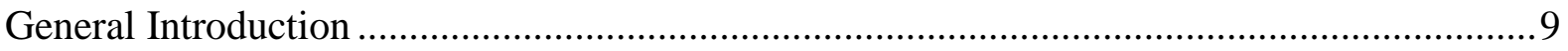

Characteristics and prevalence of Non-Suicidal Self-Injury .............................................

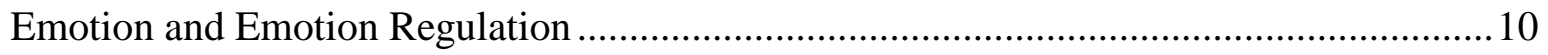

Process Model of Emotion Regulation .................................................................... 11

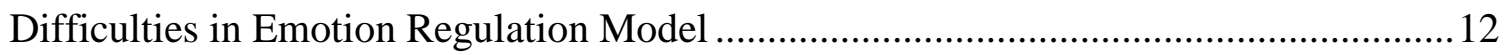

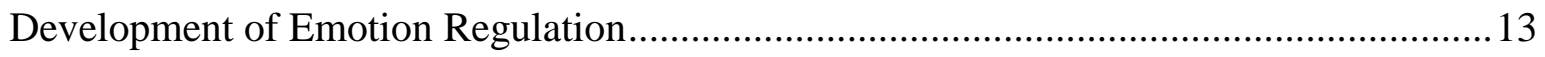

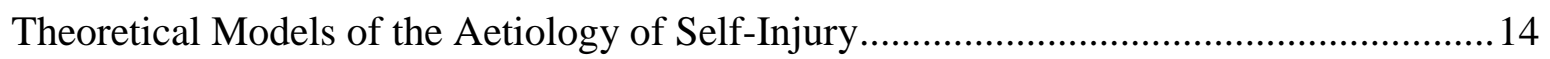

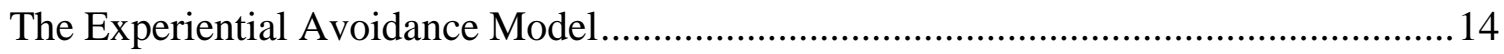

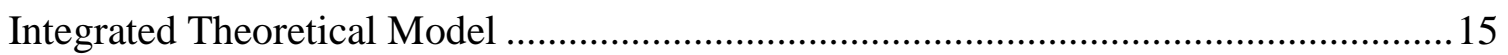

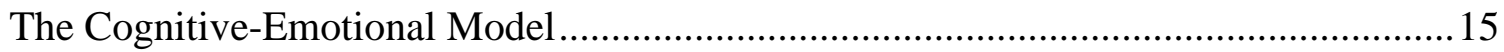

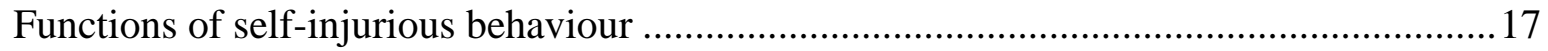

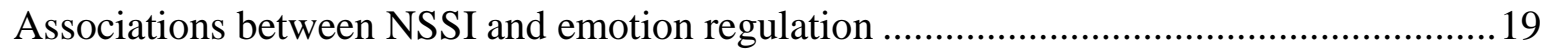

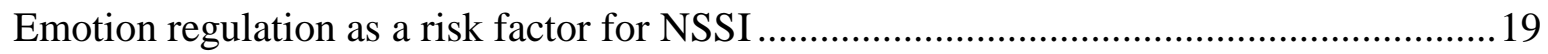

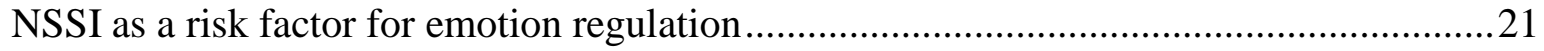

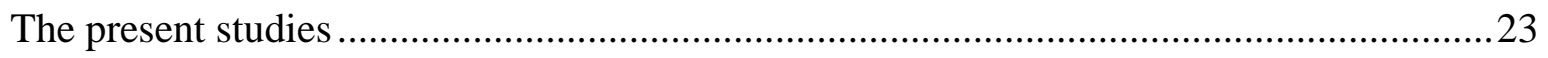

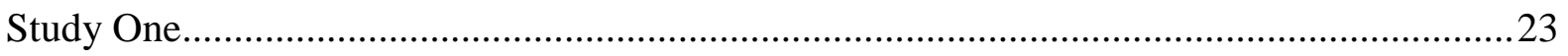

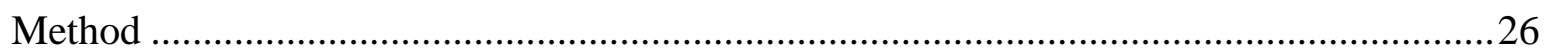

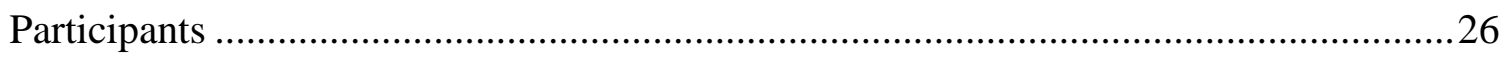

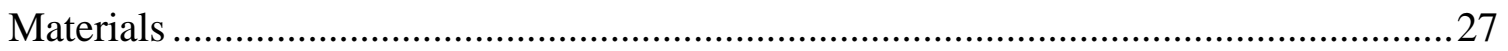

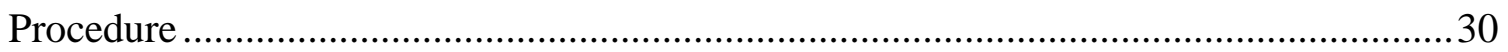

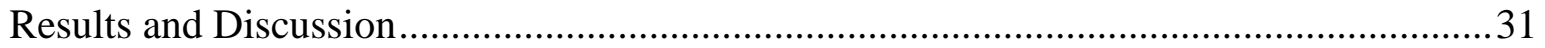




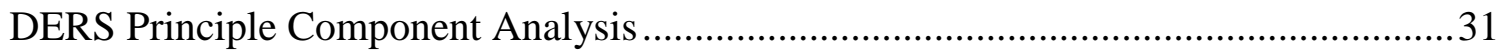

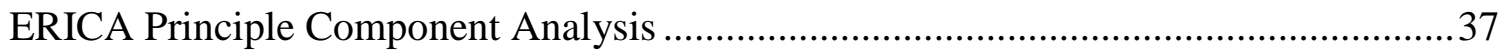

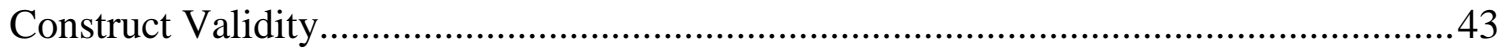

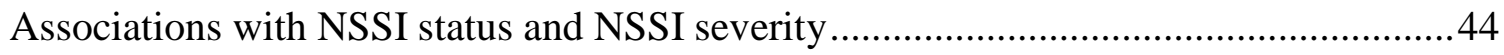

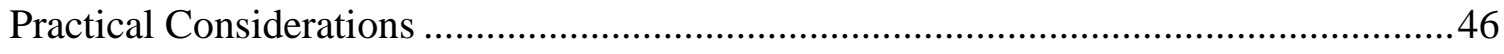

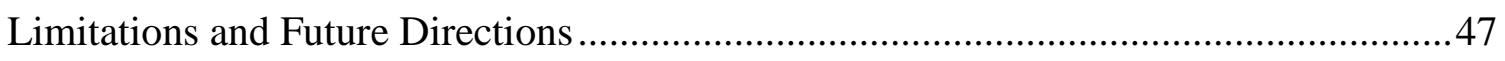

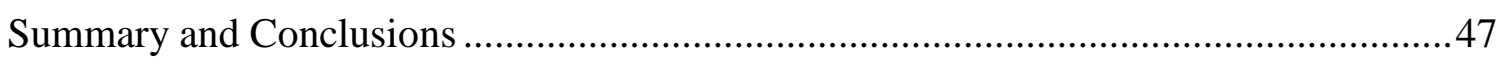

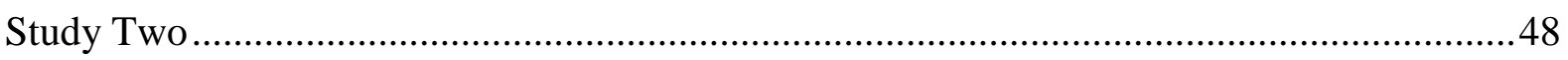

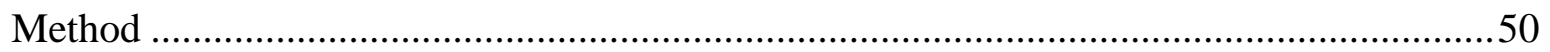

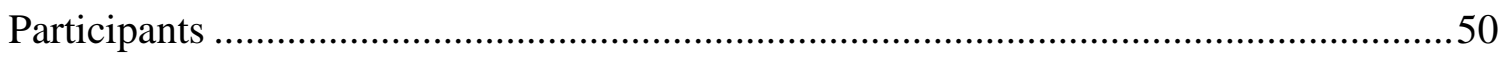

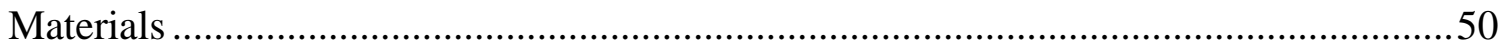

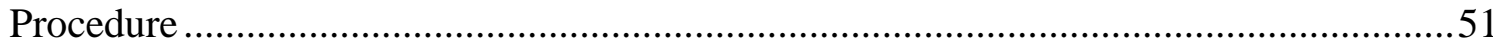

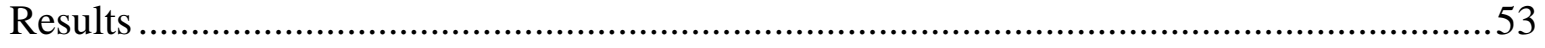

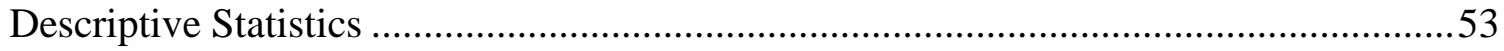

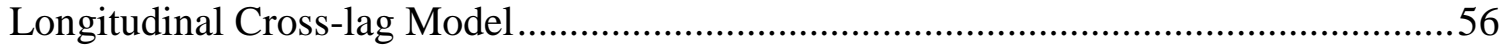

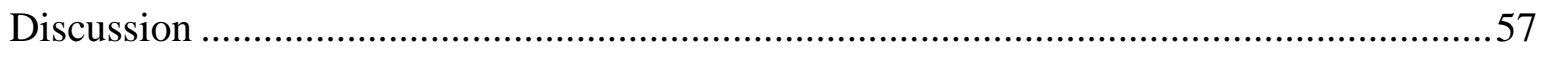

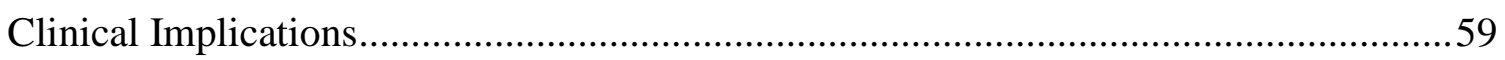

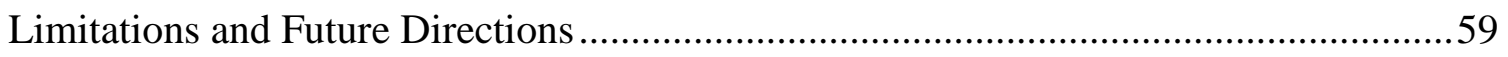

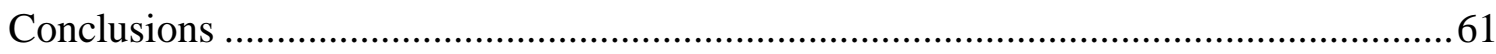

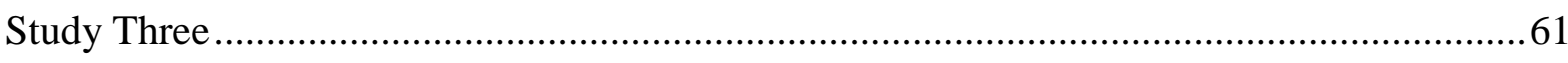

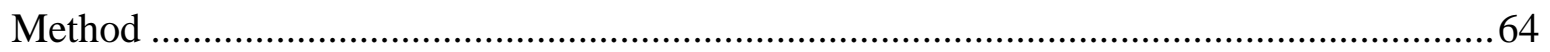

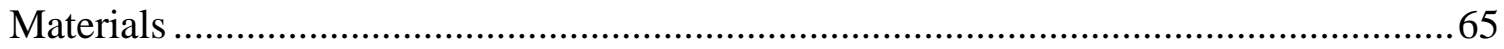

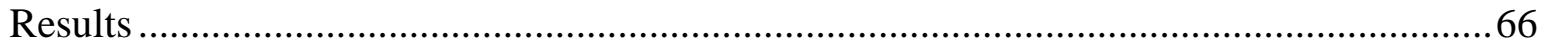

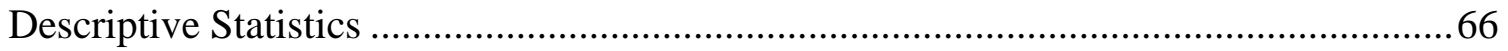

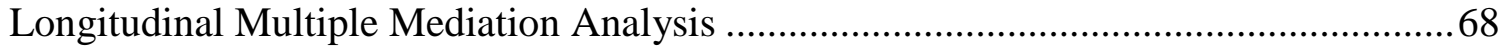

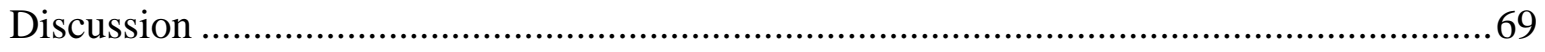

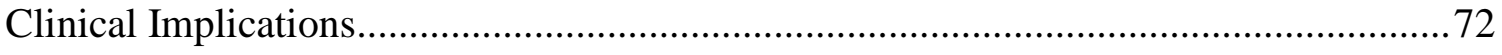




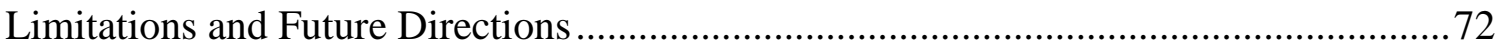

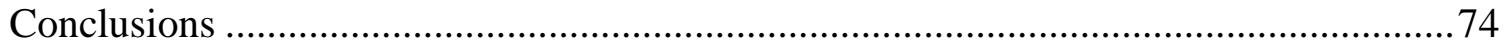

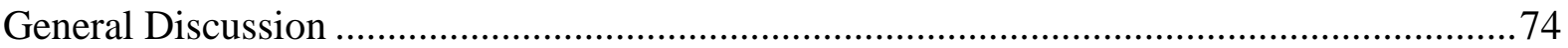

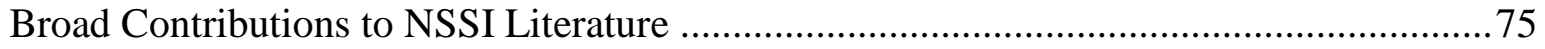

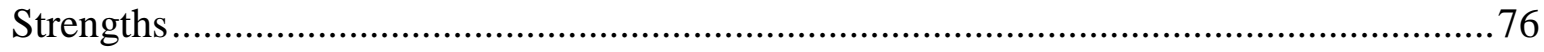

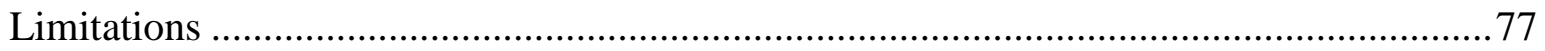

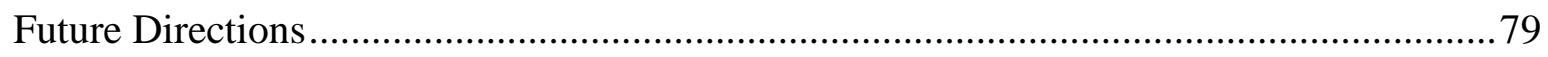

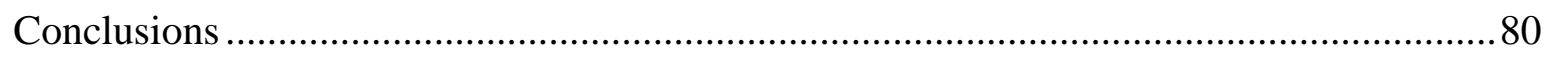

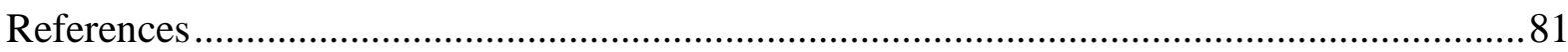

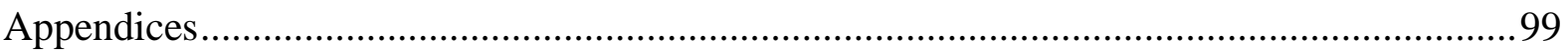




\section{List of Figures}

Figure 1. The Process Model of Emotion Regulation (Gross, 1998) showing the personsituation context and each of the five key processes of emotion regulation.

Figure 2. The Experiential Avoidance Model of Deliberate Self-Harm (Chapman et al., 2006)

Figure 3. The Integrated Theoretical Model of Non-Suicidal Self-Injury (Nock, 2010) 15

Figure 4. The Cognitive-Emotional Model of Non-Suicidal Self-Injury (Hasking, Whitlock, Voon, \& Rose, 2016)

Figure 5. Scree plot displaying both the observed eigenvalues generated from the initial Principal Components Analysis and the random eigenvalues generated by the parallel analysis, for each of the 36 possible Difficulties in Emotion Regulation Scale components in Study One

Figure 6. Scree plot displaying the observed eigenvalues generated from the initial Principal Components Analysis as well as the random eigenvalues generated from the parallel analysis, for each of the possible 15 components of the Emotion Regulation Index for Children and Adolescents in Study One .......................................................... 38

Figure 7. Proposed cross-lagged model in Study Two ….....................................................55

Figure 8. Standardised regression weights for the cross-lag model in Study Two..................55

Figure 9. Proposed multiple mediation model in Study Three.

Figure 10. Parameter estimates for the direct and indirect effects within multiple mediation model in Study Three 


\section{List of Tables}

Table 1. Results of Parallel Analysis used to determine the most appropriate number of components within the Difficulties in Emotion Regulation Scale in Study One .....32

Table 2. Items comprising the Difficulties in Emotion Regulation Scale with factor loadings in Study One

Table 3. Descriptive statistics for the Difficulties in Emotion Regulation Scale total and subscale scores split by gender in Study One.....

Table 4. Results of Parallel Analysis used to determine the most appropriate number of components within the Emotion Regulation Index for Children and Adolescents in Study One.

Table 5. Items comprising the Emotion Regulation Index for Children and Adolescents with factor loadings in Study One.

Table 6. Correlations between the Emotion Regulation Index for Children and Adolescents and the Difficulties in Emotion Regulation Scale total scores and subscales in Study One.

Table 7. Descriptive statistics for Emotion Regulation Index for Children and Adolescents total and subscales scores, split by gender in Study One

Table 8. Zero-order correlations between the Emotion Regulation Index for Children and Adolescents, the Difficulties in Emotion Regulation Scale, Depression, Anxiety, and Self-Esteem in Study One

Table 9. Zero-order correlations ( $r$ s) between gender, NSSI and Emotion Regulation across the three time points in Study Two.

Table 10. Zero-order correlations between T1 NSSI Severity, T2 Parental Attachment, T2

Peer Attachment, and T3 Emotion Regulation in Study Three

Table A. Endorsement of different NSSI behaviours among participants who reported engaging in NSSI in Study One

Table B. Functions of NSSI among participants who reported engaging in NSSI in Study One

Table C. Lifetime endorsement of different NSSI behaviours among participants who reported engaging in NSSI across three waves in Study Two and Three

Table D. Lifetime endorsement functions of NSSI among participants who reported engaging in NSSI across three waves in Study Two and Three. 


\section{Foreword}

This thesis developed out of my work as part of the Youth Wellbeing Study team. The Youth Wellbeing Study is a research project which investigates the wellbeing of young New Zealanders and rangatahi, with a specific focus on non-suicidal self-injury. The study programme began in 2012 and is comprised of a qualitative component that involved interviewing young people and the adults who support them, and a quantitative component in the form of a four-wave longitudinal survey and a comparison cross-sectional sample. Since my involvement in the research team began in late 2013, I have been involved in all aspects of the project: ethics applications, transcription of interviews, survey design, liaising with schools and parents, data collection at the 16 participating schools, data management and archiving, community presentations, and the development of psychoeducation resources. I have also been responsible for the day-to-day running of the project for several months when the Post-Doctoral Follow was on parental leave. Indeed, I've been involved in collecting the longitudinal and cross-sectional data presented in this thesis, before enrolling in the Masters by thesis programme. My thesis involved the analyses and interpretation of the longitudinal data to test specific hypotheses about the relationship between emotion regulation and NSSI.

Further information about the Youth Wellbeing Study can be found at: youthwellbeingstudy.wordpress.com. 


\section{Reciprocal risk:}

The longitudinal relationship between emotion regulation and non-suicidal self-injury

Internationally, approximately one in five adolescents have, without suicidal intent, deliberately engaged in behaviours that directly injure their bodily tissue (Muehlenkamp, Claes, Havertape, \& Plener, 2012; Swannell, Martin, Page, Hasking, \& St John, 2014). This Non-Suicidal Self-Injury (NSSI) is associated with poor psychological wellbeing, such as depression (Garisch \& Wilson, 2015), eating disorders (Muehlenkamp, Claes, Smits, Peat, \& Vandereycken, 2011), and risk of subsequent suicide (Guan, Fox, \& Prinstein, 2012); all of which carry substantial long-term individual, social and economic costs (see for example, Knapp \& McDaid, 2009; Luppa, Heinrich, Angermeyer, König, \& Riedel-Heller, 2007; Shepard, Gurewich, Lwin, Reed, \& Silverman, 2016). Within New Zealand specifically, the economic costs of self-injury and suicide are over two billion dollars per year (O'Dea \& Wren, 2010).

Converging evidence from personal narratives, ecological momentary assessment, and experiments using pain manipulations as a proxy, demonstrates that NSSI functions to manage overwhelming emotional experiences (Edmondson, Brennan, \& House, 2016; Franklin et al., 2010; Muehlenkamp et al., 2009; Nock, Prinstein, \& Sterba, 2009). Coupled with extensive cross-sectional research showing an association between emotion regulation and NSSI, this pattern of results suggests that a deficit in emotion regulation skills may underlie NSSI. However, at present longitudinal research assessing the relationship between NSSI and emotion regulation during adolescence is limited, curtailing our ability to empirically test the causal mechanisms fundamental to the development of NSSI.

\section{Characteristics and prevalence of Non-Suicidal Self-Injury}

Although a relatively young field of inquiry, many different terms have been used to describe self-injury, including "non-suicidal self-injury”, "deliberate self-harm”, "parasuicide", and "self-mutilation" (see for example, Brown, Comtois, \& Linehan, 2002; Favazza \& Conterio, 1988; Hawton, Rodham, Evans, \& Weatherall, 2002; Whitlock, Eckenrode, \& Daniel, 2006). Currently, there are two main definitions in use; Deliberate Self-Harm (DSH) and Non-Suicidal Self-Injury (NSSI). Some researchers argue that selfinjury lies along a continuum of severity, ranging from superficial cutting and scratching of skin, to suicide and self-amputation, and so caution against including motivation or intent within a definition (see for example, Hawton, Rodham, \& Evans, 2006; Stanley, Winchel, Molcho, Simeon, \& Stanley, 1992). As such, DSH refers to intentional and self-directed 
behaviours to damage body tissue, such as cutting of skin, self-poisoning, or suicidal behaviour (Hawton, Saunders, \& O’Connor, 2012). However, NSSI can be distinguished from suicidal behaviours in terms of lethal intent, method severity, frequency, commitment to life, and consequences (Muehlenkamp \& Kerr, 2010; see also Butler \& Malone, 2013; Klonsky \& Olino, 2008). Therefore, this thesis explicitly focuses on non-suicidal self-injury defined as the deliberate and self-inflicted destruction of body tissue without suicidal intent, and for purposes not socially-sanctioned (International Society for the Study of Self-Injury, 2017; Nock, 2009).

NSSI is relatively common within community samples (Klonsky, 2011; Whitlock et al., 2006), particularly among adolescents - international reviews suggest that across cultures approximately $18 \%$ of adolescents report a lifetime history of NSSI (Muehlenkamp et al., 2012; Swannell et al., 2014). The onset of NSSI appears to range from 12 to 15 years old, with NSSI engagement peaking at around 15 and 16 years old, before declining in early adulthood (for a review, see Plener, Schumacher, Munz, \& Groschwitz, 2015). However it is important to note that NSSI is a highly variable behaviour; within a young adult sample $25 \%$ of participants with a history of NSSI reported engaging in NSSI only once while $15 \%$ had engaged in NSSI more than 20 times (Whitlock et al., 2006). Common NSSI behaviours include cutting or scratching skin, and self-bruising (Bjärehed \& Lundh, 2008; Whitlock, Eckenrode, \& Daniel, 2006). Girls may be slightly more likely to engage in NSSI than boys (Plener, Libal, Keller, Fegert, \& Muehlenkamp, 2009; although see Garisch \& Wilson, 2015), particularly in stereotypical NSSI behaviours such as cutting or scratching, while boys are more likely self-injure by burning or punching themselves (Andover, Primack, Gibb, \& Pepper, 2010; Whitlock et al., 2011).

\section{Emotion and Emotion Regulation}

Given the importance of emotion regulation in understanding NSSI, before reviewing the NSSI literature it is critical to establish a working framework of emotion regulation. Emotions are multifaceted phenomena consisting of changes in subjective feelings, behaviour (also called action urges), and physiology (Mauss, Levenson, McCarter, Wilhelm, \& Gross, 2005), that arise when an individual attends to an internal or external stimulus and appraises it as relevant to their goals (Gross \& Thompson, 2007). Phenomenologically, emotions operate along axes of arousal, ranging from high intensity to low intensity, and valence, ranging from the positive to negative (Bradley \& Lang, 1994). In conjunction, arousal and valence comprise a large range of discrete emotional experiences, ranging from 'basic' 
emotions such as disgust, happiness or fear (Ekman, 1992) to highly complex emotional experiences such as empathy, nostalgia or schadenfreude (Bendelow \& Williams, 1998). Emotions are functional in that they motivate us to action and enable us to respond to our environment (Bradley \& Lang, 2007). Although emotions have "control precedence" in that they interrupt and capture our attention (Frijda, 1986, as cited in Gross \& Thompson, 2007), emotions are also malleable. Emotion regulation is the process by which we mould our emotions, defined within this thesis as the behaviours, skills, and strategies, whether automatic or effortful, which function to modulate, inhibit, and enhance emotional experiences and expressions (see Gross \& Thompson, 2007, for a review). It is important to note that emotion regulation processes are used to increase or decrease both positive and negative emotions. In particular, the NSSI literature draws strongly from two frameworks of emotion regulation.

\section{Process Model of Emotion Regulation}

The Process Model of Emotion regulation (Gross, 1998; Gross, 2015) is a widely used framework for understanding emotion regulation. A key aspect of this model is that emotions unfold over time within a person-situation context. Different emotion regulation processes are employed at different time periods (see Figure 1). These processes can be split into antecedent-focused strategies which regulate an emotion before it is generated (e.g., choosing not to watch news coverage of a natural disaster), and response-focused strategies which regulate an emotion as it is generated (e.g., breathing deeply to reduce heart rate). Two specific emotion regulation strategies which have been the focus of much empirical investigation are cognitive reappraisal and expressive suppression (see for example, McRae et al., 2012; Tatnell, Kelada, Hasking, \& Martin, 2014). Cognitive reappraisal refers to a change in how the situation is perceived, in a way that changes its emotional impact (Gross, 1998; Lazarus \& Alfert, 1964). For example, when viewing gory images a person may choose to think that they are photographs of movie props rather than the aftermath of a train crash. In comparison, expressive suppression is a response modulation process which focuses on inhibiting emotional expression (Gross, 1998). For example, after winning a game a person may prevent themselves from grinning widely in order to maintain friendships. Previous research has established meaningful individual differences in emotion regulation strategy use (see for example, Gross \& John, 2003). In a meta-analytic review, Aldao, NolenHoeksema, and Schweizer, (2010) found that emotional avoidance and expressive 

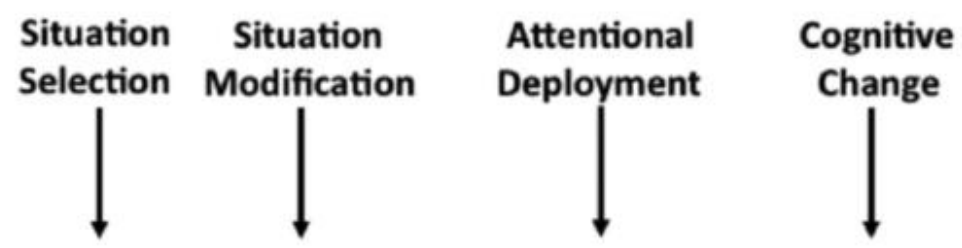

Response

Modulation
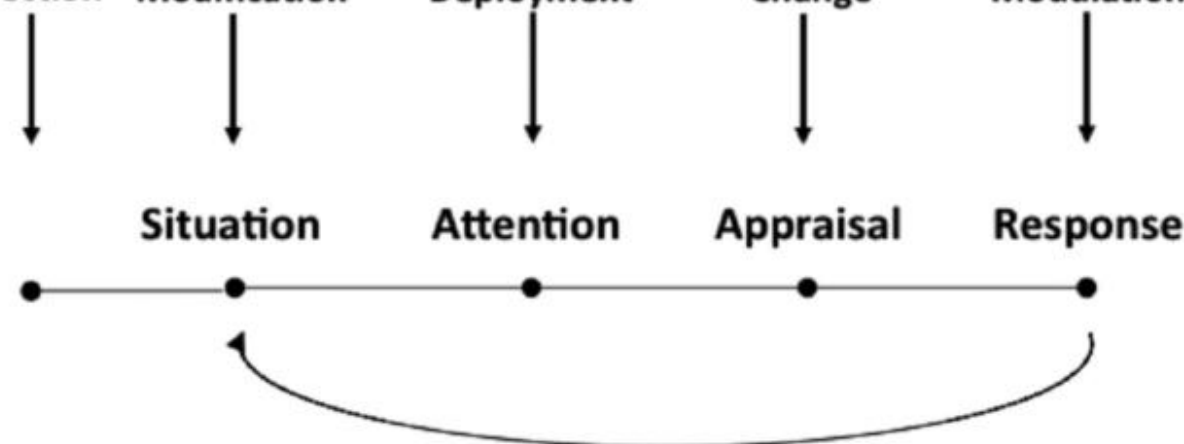

Figure 1. The Process Model of Emotion Regulation (Gross, 1998) showing the personsituation context and each of the five key processes of emotion regulation.

suppression were associated with greater psychopathology whereas problem-solving and cognitive reappraisal were associated with lower psychopathology.

The argument that emotion and emotion regulation unfold simultaneously over time has implications for how we understand emotional reactivity - an individual's threshold for an emotional response, and the intensity and duration of that response (Davidson, 1998). Although emotion reactivity can be conceptually distinguished from emotion regulation, the process model argues that antecedent-focused emotion regulation processes may change the quality of the emotion before it is generated and so appear to 'decrease' reactivity. For instance, Williams, Bargh, Nocera and Gray (2009) demonstrated that priming cognitive reappraisal outside of awareness, prior to a mood induction, reduced physiological reactivity to the same extent as did explicit instructions. That is, unconscious emotion regulation affected subsequent emotional reactivity. Given that the dynamic interplay between emotional reactivity and regulation makes it difficult to distinguish one from the other, this thesis therefore considers emotional reactivity to be part of the emotion regulation process.

\section{Difficulties in Emotion Regulation Model}

In contrast to the Process Model of Emotion Regulation, the Difficulties in Emotion Regulation Model argues that, in addition to understanding the processes by which we regulate our emotions, emotional awareness and acceptance are critical to functional emotion regulation (Gratz \& Roemer, 2004). Emotion dysregulation occurs when habitual emotion regulation patterns impair functioning by disrupting attention, interpersonal relationships, or fail to flexibly regulate emotional experiences (Cole, Michel, \& Teti, 1994). The Difficulties 
in Emotion Regulation Model grew out of theoretical work identifying emotion dysregulation as the core characteristic of borderline personality disorder, which arises from biological vulnerabilities in the context of an invalidating family environment (Linehan, 1993). Gratz and Roemer's (2004) model argues that awareness and understanding of emotions, acceptance of emotions, the ability to control impulses and behaviour during an emotional response, and the ability to flexibly use situationally appropriate strategies are key factors which underlie effective emotion regulation. Critically, this framework takes an individual differences approach to better understand the role of emotion regulation in psychopathology. The Difficulties in Emotion Regulation Scale (DERS; Gratz \& Roemer, 2004) is a self-report measure of emotion dysregulation which draws directly from this model. Previous research with the DERS has linked greater emotion dysregulation with greater psychopathology (see for example Tull, Barrett, McMillan, \& Roemer, 2007; Whiteside et al., 2007), including NSSI (see for example, Gratz \& Tull, 2010; Weinberg \& Klonsky, 2009).

Although the Process Model and the Difficulties in Emotion Regulation Model provide relatively different conceptualisations of emotion regulation, it is important to note the models are not mutually exclusive. Although there is value in differentiating the processes from the traits, both of these aspects are strongly integrated and interrelated within the phenomenon of emotion regulation. In fact, a recent theoretical model of NSSI draws heavily on both frameworks (Hasking, Whitlock, Voon, \& Rose, 2016). Therefore, in this thesis I draw from both frameworks.

\section{Development of Emotion Regulation}

Adolescence is a critical time for the development of emotion regulation skills. Relative to childhood, adolescents experience an increased need to regulate emotions to achieve long term goals (Steinberg, 2005), as well as number of novel emotional situations such as working to establish their personal identity, beginning (and ending) romantic relationships, and increasing school assessment. During early adolescence, puberty triggers heightened emotion arousal, sensation seeking and motivation, followed by maturation of the frontal lobes which underlie self-regulation in late adolescence (Steinberg, 2005). Substantial brain maturation during adolescence underlies changes in executive functioning, social cognition, perception and evaluation of risk and reward, as well as the regulation of emotion and behaviour (McRae et al., 2012; Paus, 2005; Steinberg, 2005). In addition to maturation processes, the emotional landscape of the family environment directly affects the interactional style a young person has with their peers (Eisenberg et al., 2001; Engels, Rutger, 
Finkenauer, Meeus, \& Deković, 2001), and therefore their emotional wellbeing. During adolescence, young people shift from primarily external toward internal self-regulation strategies, and in doing so experiment with different strategies (Baumrind, 1987). NSSI is argued to be one such emotion regulation strategy (see for example, Chapman, Gratz, \& Brown, 2006)

\section{Theoretical Models of the Aetiology of Self-Injury}

Within the literature there are well-established models of the development of NSSI. I now present a brief summary of three of these models, not to draw comparisons between them, but rather to demonstrate the critical role emotion regulation plays in our current theoretical understandings of NSSI.

\section{The Experiential Avoidance Model}

The Experiential Avoidance Model (Chapman et al., 2006) provides a framework for understanding how self-injurious behaviours are reinforced and maintained over time via emotional avoidance processes (see Figure 2). Psychological risk factors, such as a deficit in emotion regulation skills or poor distress tolerance, prompt an overwhelming emotional response to a stimulus. The person then avoids this aversive emotional response by engaging

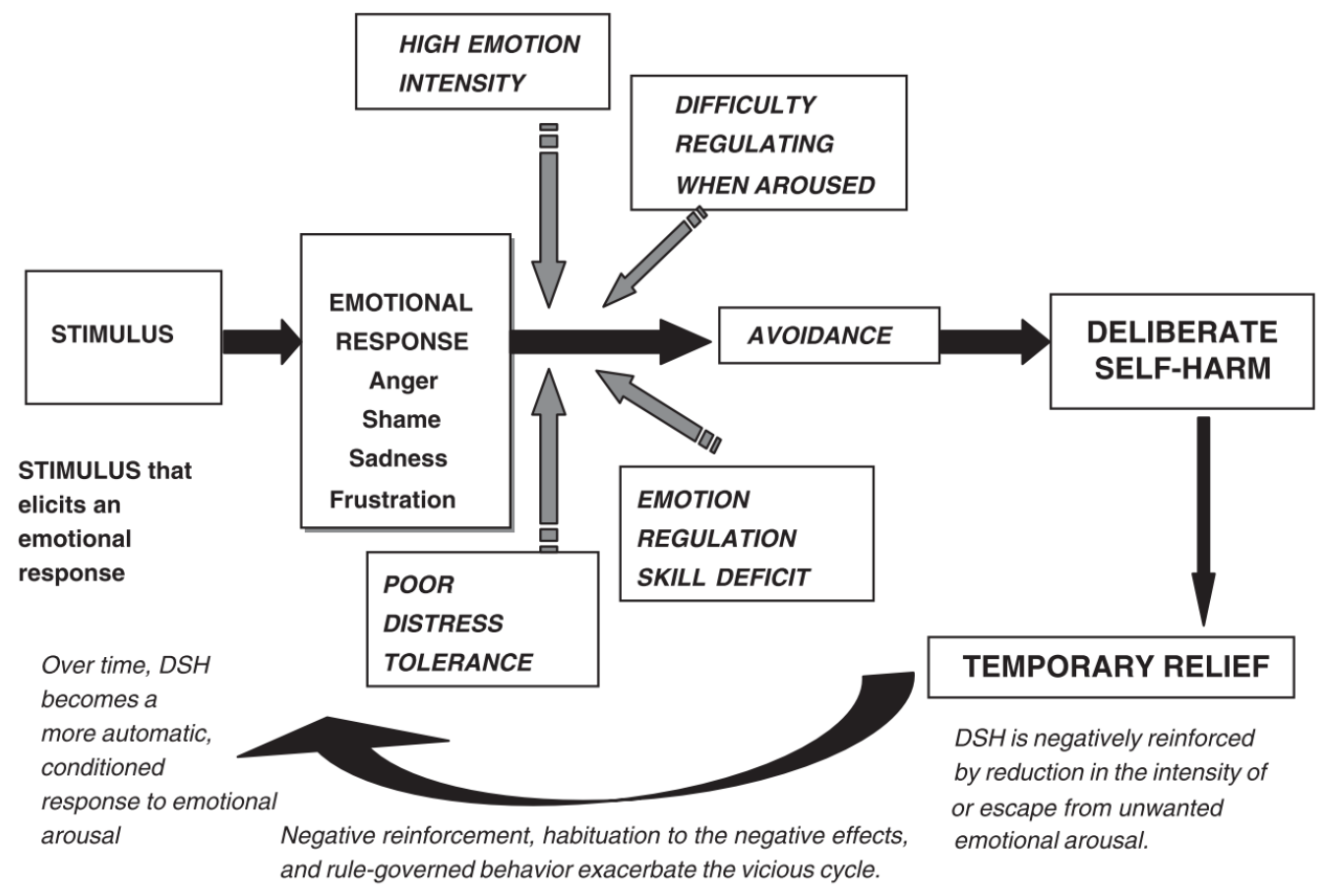

Figure 2. The Experiential Avoidance Model of Deliberate Self-Harm (Chapman et al., 2006). 
in self-injury (e.g., through distraction, or self-punishment) which leads the behavior to be negatively reinforced. Over time, an individual habituates to the constraining factors (e.g., fear of pain) and self-injury becomes a habitual strategy to manage unwanted emotions.

\section{Integrated Theoretical Model}

In contrast, the Integrated Theoretical Model of NSSI (Nock, 2010) provides a framework for understanding how different risk factors interact to create NSSI onset (see Figure 3). This model suggests that distal risk factors, such as familial criticism and a genetic predisposition for high emotional reactivity, leads to both intrapersonal and interpersonal vulnerability factors such as more frequent aversive emotions and poor communication skills which creates a hypo- or hyper-aroused stress response. In the context of NSSI specific vulnerability factors, such as self-punishment beliefs, this altered stress response causes an individual to engage in NSSI to regulate their emotion experience or social situation. In contrast with the Experiential Avoidance Model, Nock's (2010) Integrated Theoretical Model argues that NSSI functions not only to down-regulate aversive emotions, but also to up-regulate low arousal emotions (e.g., to prevent emotional numbness).

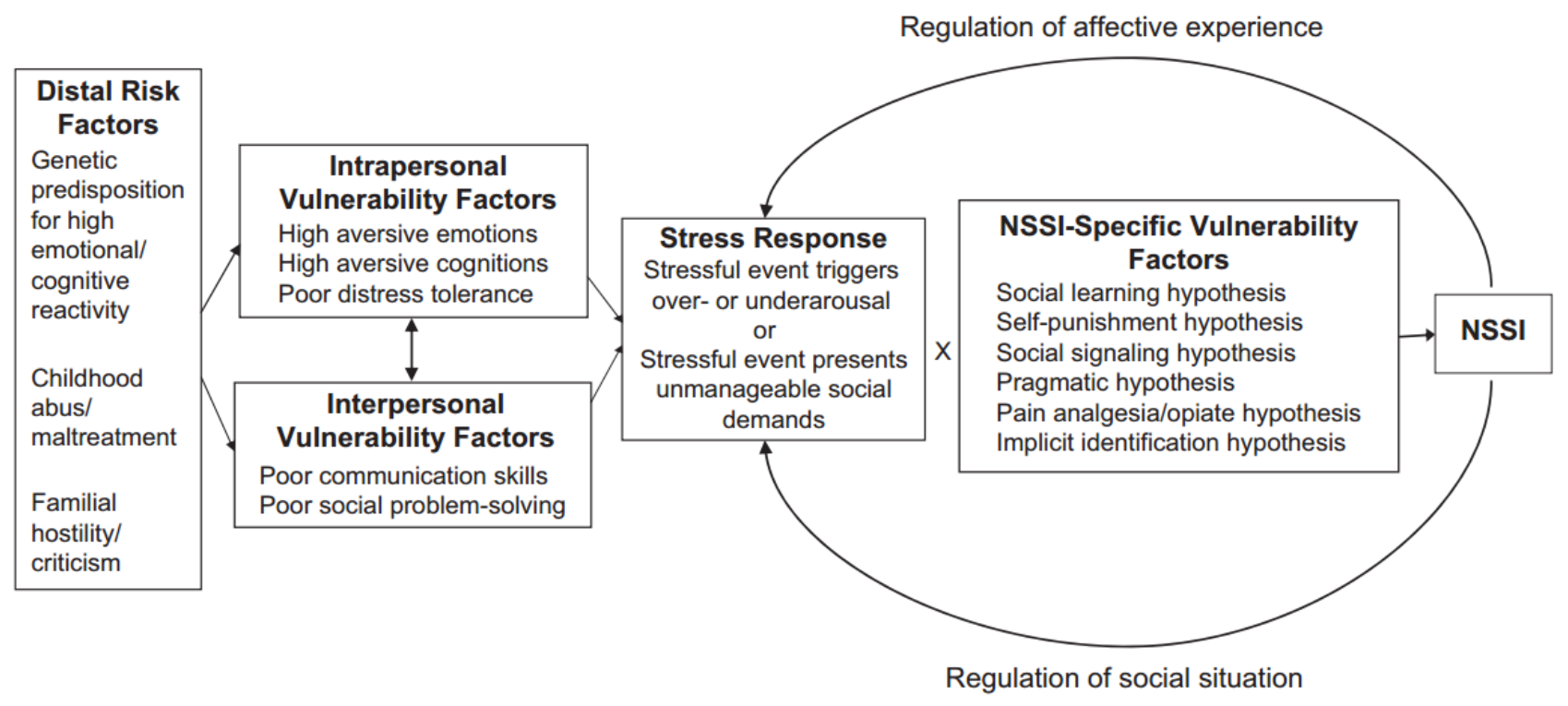

Figure 3. The Integrated Theoretical Model of Non-Suicidal Self-Injury (Nock, 2010).

\section{The Cognitive-Emotional Model}

In comparison to both the Experiential Avoidance Model and the Integrated Theoretcial Model, the Cognitive-Emotional Model of NSSI (Hasking, Whitlock, Voon \& 
Rose, 2016) emphasises the dual role of emotion regulation and self- and NSSI-related cognitions in the development of NSSI (see Figure 4). Individuals have a tendency towards emotional high reactivity which interacts dynamically with; their representations of NSSI (e.g., 'cutting is something only girls do'), NSSI-related cognitions (e.g., 'physical pain helps to release my emotional pain'), and their self-representations (e.g., 'I'm a terrible person who deserves to be punished'). Within the context of an intense emotional response, the combination of these emotional and cognitive risk factors leads to NSSI either to avoid the emotion, avoid the situation, or to modulate their emotional response. Compared to the Experiential Avoidance Model and the Integrated Theoretical Model, the CognitiveEmotional Model argues that these NSSI-related cognitions determine why people choose to engage in NSSI rather than other experimental avoidance behaviours such as drug or alcohol use.

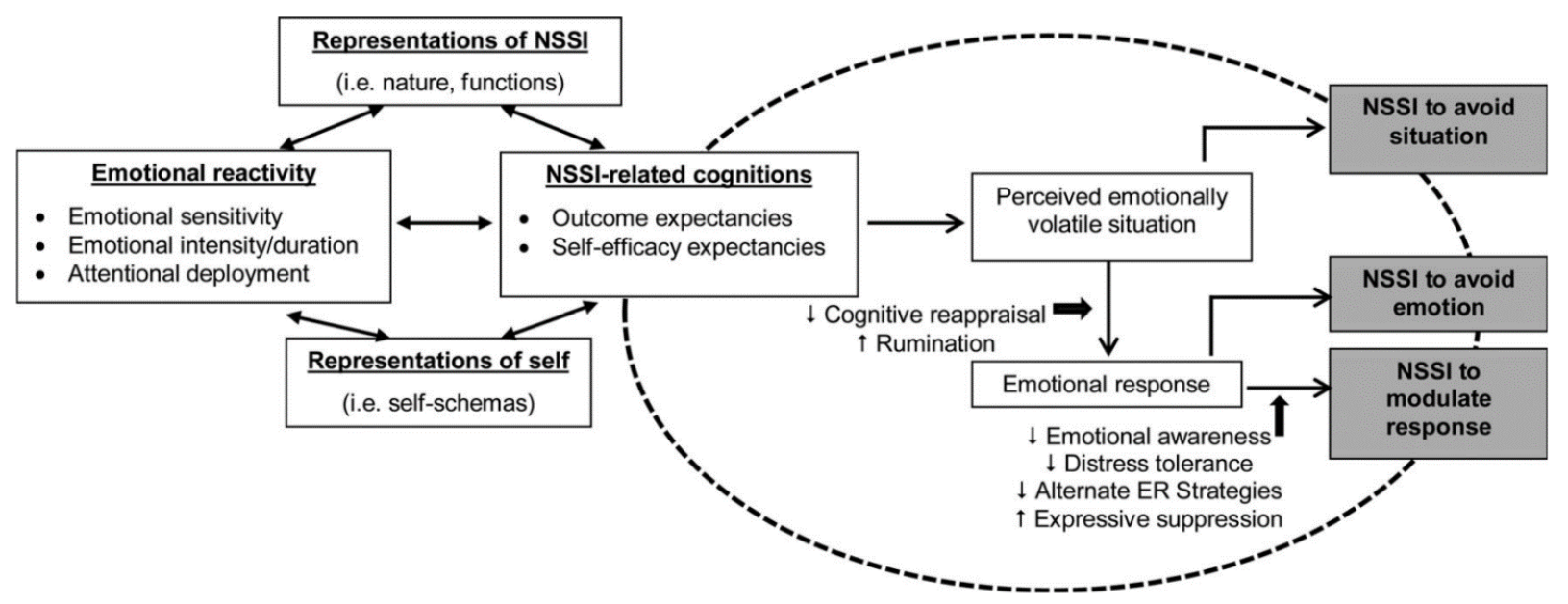

Figure 4. The Cognitive-Emotional model of Non-Suicidal Self-Injury (Hasking, Whitlock, Voon, \& Rose, 2016).

Although the models reviewed differ in complexity and ability to predict nuanced NSSI behaviour, it is important to note that each emphasises the importance of the emotional response, and the regulation of that response in understanding self-injury. Drawing from the Difficulties in Emotion Regulation Model, all three models highlight deficits in characteristics underlying functional emotion regulation, such as emotional awareness and distress tolerance. Drawing from the Process Model of Emotion Regulation, the CognitiveEmotion Model also highlights the importance of emotion regulation processes such as 
cognitive reappraisal, rumination and emotional expression. Together, all three of these models of self-injury emphasise emotion regulation deficits, providing a strong and consistent theoretical argument that emotion regulation underlies the development of NSSI.

\section{Functions of self-injurious behaviour}

Empirical evidence for the importance of emotion regulation in NSSI comes first from functional analyses of self-injury. The functional perspective of NSSI emphasises that individuals who engage in self-injury do so because the behaviour serves one or more function(s) that are not otherwise currently met. By fulfilling these functions the behaviour is either positively or negatively reinforced leading to the maintenance and escalation of selfinjury over time (Klonsky, 2007). In contrast, removing the reinforcement or creating alternative strategies to meet these functions leads to reduction or cessation of self-injury (Klonsky \& Glenn, 2009; Nock \& Prinstein, 2004). By identifying the functions that an individual's NSSI serves, we can broadly infer the psychological difficulties a person may be experiencing. Both theoretical and empirical work has grouped the functions of NSSI into two superordinate clusters; Intrapersonal Functions associated with regulating internal thoughts and feelings, and Interpersonal Functions concerning management of social relationships (Klonsky \& Glenn, 2009; Nock \& Prinstein, 2004).

The Inventory of Statements About Self-Injury deconstructs these two superordinate clusters into 13 different functions, derived both from an empirical review of the evidence, and discussion with treatment professionals, researchers, and consumers (Klonsky \& Glenn, 2009). The Interpersonal cluster is comprised of Autonomy, Interpersonal Boundaries, Interpersonal Influence, Peer-Bonding, Revenge, Self-Care, Sensation Seeking, and Toughness functions. The Intrapersonal cluster is comprised of Affect-Regulation, AntiDissociation, Anti-Suicide, Marking Distress, and Self-Punishment functions. At face value, the Affect Regulation function maps onto theoretical conceptualisations of emotion regulation. Affect Regulation scores are calculated as an individual's average response to items which describe NSSI as an emotion regulation strategy, such as 'When I self-injure, I am reducing anxiety, frustration, anger, or other overwhelming emotions'. International (Bildik, Somer, Kabukçu Başay, Başay, \& Özbaran, 2013; Klonsky \& Glenn, 2009; Kortge, Meade, \& Tennant, 2013) and local research (Langlands, 2012) demonstrates that Affect Regulation is the most strongly and commonly endorsed function of NSSI. In a meta-analysis of 152 studies assessing self-reported reasons for NSSI, the majority of both questionnaire (93\%) and interview based (92\%) studies endorsed NSSI as a strategy to manage distress 
(Edmondson et al., 2016). Regardless of sample type (community vs clinical vs forensic, adolescent vs. adult, women vs men), or methodology (self-report measure or personal narratives), there is considerable evidence that for many people, self-injury is primarily a strategy with which to manage their emotions (Edmondson et al., 2016; Klonsky, 2007). Converging evidence that NSSI functions to temporarily reduce negative affect comes from personal narratives (Edmondson et al., 2016), ecological momentary assessment studies (Muehlenkamp et al., 2009), as well as laboratory-based physiology studies with pain manipulations as NSSI proxies (Franklin et al., 2010).

However, it is likely that many of the other 12 NSSI functions measured by the ISAS also indirectly tap into emotion regulation processes. Self-Punishment for instance, often the second most endorsed function (Klonsky \& Glenn, 2009; Martin, Swannell, Hazell, Harrison, \& Taylor, 2010), enables an individual to express or enact self-directed emotions such as shame, anger or frustration, and so reduces the intensity or magnitude of that emotional response. NSSI also functions to prevent numbness or dissociation, allowing the person to create or intensify an emotional response (for a similar argument, see Mckenzie \& Gross, 2014; Prinstein, 2008). Even the functions classified as interpersonal contain an emotional response to be regulated; for instance, if one didn't have an emotional reaction to a romantic relationship ending, one wouldn't want to exact revenge. NSSI may therefore allow the individual to manipulate their emotional experience to serve a variety of functions.

Although functional assessment of NSSI highlights the importance of emotion regulation in maintaining the behaviour, a reliance on functional explanations is limited in several key ways. First, I argue that the reasoning that "people with NSSI have difficulties with emotion regulation because they engage in NSSI as an emotion regulation strategy' is circular. Secondly, a functional perspective is likely to underestimate the degree of impairment as it does not take into account other behaviours common among people who self-injure, such as substance use or maladaptive eating (Hilt, Nock, Lloyd-Richardson, \& Prinstein, 2008), which may serve similar functions (see Muehlenkamp et al., 2009 for a similar argument). Finally, people without NSSI are unable to report the reasons for their non-existent self-injury. Therefore, relying only on NSSI functional assessment prevents any comparison with a control group, as well as severely curtailing our ability to track the development and trajectory of NSSI. 


\section{Associations between NSSI and emotion regulation}

If poor emotion regulation underlies NSSI, then people with NSSI should report poorer emotion regulation than people without NSSI. Perez, Venta, Garnaat, and Sharp (2012) found that inpatients presenting with NSSI reported greater trait emotion dysregulation than did inpatients without NSSI, even when controlling for gender and comorbid psychopathology. Within young adult community samples (which likely better capture the full spectrum of NSSI severity), people who engage in NSSI consistently report greater emotion dysregulation than do their peers who do not self-injure (Eichen et al., 2015; Muehlenkamp, Kerr, Bradley, \& Larsen, 2010). This group-level difference is also evident within community adolescent samples, where adolescents who self-injure are 3.3 times more likely to report difficulty using cognitive reappraisal strategies (but report no differences in emotional suppression) compared to their peers (Martin et al., 2010). Moving beyond predicting NSSI status, research taking into account the heterogeneity of NSSI behaviours has found emotion dysregulation is also associated with greater NSSI severity (Gratz \& Roemer, 2004; Yurkowski et al., 2015). In terms of specific emotion regulation processes NSSI severity shows a small negative correlation with cognitive reappraisal, and a small positive correlation with emotional suppression in community adolescent samples (Hasking et al., 2010; Williams \& Hasking, 2010). Taken together, these studies provide robust evidence that NSSI is associated with poorer self-reported emotion regulation abilities.

\section{Emotion regulation as a risk factor for NSSI}

However, it is critically important to distinguish between correlates of NSSI and risk factors for NSSI. We may be confident that emotion dysregulation is associated with NSSI, but the specific nature of this association is unknown. The vast majority of this research is cross-sectional in nature, and so it is not clear if poor emotion regulation causes NSSI, NSSI causes poor emotion regulation, or if both poor emotion regulation and NSSI are caused by an unknown third variable.

Primary evidence that emotion regulation may play a causal role in NSSI comes from studies comparing people with a current history of NSSI to those with a past history. In an inpatient sample, Gratz, Breetz, and Tull (2010) found that there was no difference in reports of emotion dysregulation between patients reporting recent DSH and those reporting historic DSH. However, with 9 participants in each group this study is highly likely to be underpowered. Whitlock, Prussien, and Pietrusza (2015) recruited a large sample of university students with a history of NSSI $(N=490)$, of whom $58.6 \%$ reported current NSSI 
(within the past 12 months) and 41.4\% reported historic NSSI (over 12 months prior, and who also reported that were unlikely to ever self-injure again). Individuals who had stopped engaging in NSSI were 1.39 times more likely to report access to effective emotion regulation strategies than those reporting active NSSI, and were also more likely to perceive greater social support, sense of meaning in life, and life satisfaction. Participants who reported being confident they have stopped self-injuring were also asked why they stopped and what specifically helped them to stop. The majority (62.6\%) of participants who reported NSSI cessation identified improved emotion regulation as a part of their recovery; $38.7 \%$ indicating growth in self-awareness, and $36 \%$ indicating that connections with others played a part in their recovery.

Converging evidence for the importance of emotion regulation in NSSI cessation comes from evaluations of Emotion Regulation Group Therapy (ERGT; Gratz \& Gunderson, 2006). ERGT draws heavily from Acceptance and Commitment Therapy (ACT; Hayes, Strosahl \& Wilson, 1999) and Dialectical Behavioural Therapy (DBT; Linehan, 1993), and was initially developed as a 16 week group therapy designed to teach women with borderline personality disorder who self-injure more adaptive ways of responding to their emotions (Gratz \& Tull, 2011). Preliminary research found that ERGT improved emotion regulation functioning; a sample of 23 women with borderline personality disorder who took part in ERGT in addition to treatment as usual reported decreases in emotion dysregulation posttreatment $\left(\eta_{\mathrm{p}}{ }^{2}=.67\right)$, with $63.2 \%$ of participants showing scores that changed by at least one standard deviation from pre- to post-treatment. Over the same period that emotion regulation improved, NSSI frequency was decreased $\left(\eta_{\mathrm{p}}{ }^{2}=.31\right)$, with $70 \%$ of participants showing a reduction in NSSI of 50\% or greater (Gratz \& Tull, 2011). A follow-up longitudinal mediation analysis demonstrated that, although there was a relationship between treatment group and change in NSSI from pre-to post-treatment, this relationship was negligible once pre- to post-treatment change in emotion dysregulation was accounted for (Gratz, Levy, \& Tull, 2012). That is, taking part in ERGT treatment (compared to treatment as usual) led to greater decreases in emotion dysregulation, which in turn led to decreases in NSSI over time. Taken together, this pattern of result suggests that improvements in emotion regulation may be a key factor in the cessation of NSSI behaviours.

However, to be established as a risk factor, emotion dysregulation needs also to prospectively predict NSSI (Kraemer et al., 1997). Longitudinal studies are therefore the gold standard in testing the role of emotion regulation in the development and trajectory of NSSI. 
One such study followed a sample of 1973 community adolescents across two years (Tatnell et al., 2014). At baseline, $8.3 \%$ of participants reported having engaged in NSSI, compared to $11.9 \%$ at follow-up. Compared to their peers who do not engage in NSSI, participants who reported onset of NSSI over the year also reported less use of cognitive reappraisal regulation strategies. However, further analysis with the same sample found that reappraisal and suppression were unrelated to the onset of NSSI over and above psychological distress (Andrews, Martin, Hasking, \& Page, 2014). Taken together, these studies suggest that emotion regulation may be a risk factor for NSSI generally, but not specifically for the onset of the behaviour.

In a recent meta-analysis, Fox and colleagues (2015) identified 16 unique samples of published prospective studies longitudinally predicting NSSI, including six samples in which emotion dysregulation was assessed as a risk factor for NSSI. Contrary to theoretical predictions emotion dysregulation was only a weak risk factor for NSSI, with an odds ratio of 1.05. By way of comparison, prior NSSI behaviour (OR of 5.95) and cluster B symptoms (e.g., borderline personality disorder, antisocial personality disorder; OR of 5.93) were the strongest risk factors for NSSI. Therefore, although emotion dysregulation is a risk factor for NSSI, it may not be the sole mechanism underlying NSSI.

However, there are a number of important limitations of this meta-analysis. First, Fox and colleagues (2015) found evidence of publication bias, suggesting the results are likely inflated estimates of the true risk size. Second, work by Andrews and colleagues (2014) and Tatnell and colleagues (2014) were incorrectly included as unique samples. Third, the study was limited by the small number of unique samples able to be included in the meta-analysis. For example, the meta-analysis risk factor of cluster B symptoms, already a broad category, only included three unique studies. Therefore, a key conclusion of this meta-analysis is that more longitudinal research on the development and trajectory of NSSI is needed.

\section{NSSI as a risk factor for emotion regulation}

Although theoretical and empirical work suggests that emotion dysregulation may be a risk factor for NSSI, the potential bidirectional relationship between them has received much less systematic exploration. Broader developmental theory in the form of systems theory emphasises that the reciprocal relationships between an individual and their environment are critical in development (Cox \& Paley, 2003; Sameroff, 1983). These relationships are dynamic in that an individual both reacts to the environment and also 
actively selects, processes and changes their environment. Over time this dynamic process may result in "problem gravitation" where two (or more) factors share an interactional causality (Bergman \& Magnusson, 1997).

In the context of NSSI, sub-optimal emotion regulation skills may lead to an individual engaging in NSSI to avoid overwhelming emotions (Chapman et al., 2006). However, emotion suppression paradoxically leads to the generation of more negative beliefs about the emotional stimulus and the individual's ability to regulate their emotions (for a review see Salters-Pedneault, Tull, \& Roemer, 2004). In addition, engaging in an act of NSSI often prompts intense self-directed negative emotions, such as shame, disgust, or guilt (LayeGindhu \& Schonert-Reichl, 2005). Therefore, engaging in NSSI in order to avoid unwanted emotions may in fact increase the experience of intense emotions, as well as reducing an individual's perceived mastery and ability to tolerate distress. That is, poor emotion regulation skills provide the context for self-injury to occur, which in turn impairs future emotion regulation.

NSSI may also be a risk factor for emotion dysregulation through an interpersonal mechanism. Engaging in NSSI may prompt negative reactions from others (see for example Klineberg, Stansfeld, \& Bhui, 2013), leading to impairments in interpersonal relationships and greater social isolation (see Gratz, 2003 for a similar argument). Social isolation impairs emotion regulation skills by creating intense emotional distress (Eisenberger \& Lieberman, 2004) and mean the absence of social support as a method to weather emotional challenge (see for example, Finch, Okun, Pool, \& Ruehlman, 1999). Additionally, adolescents learn about emotions and emotion regulation through social referencing and modelling by family and peers (Morris, Silk, Steinberg, Myers, \& Robinson, 2007), so social isolation may limit opportunities for the learning and practise necessary for optimal development. Consistent with the idea that NSSI may damage social relationships, Burke and colleagues (2015) found that for adolescent girls (but not boys), NSSI prospectively predicted interpersonally stressful life events six months later. In addition, Tatnell and colleagues' (2014) longitudinal study of high school students found cognitive reappraisal mediated the relationship between parental attachment anxiety and NSSI, suggesting poor parental attachment may lead to subsequent impairments in emotion regulation. Taken together, these two studies provide preliminary evidence that engaging in NSSI may damage social relationships, in turn impairing emotion regulation and leading to greater NSSI. However, at present the proposed reciprocal relationship between emotion regulation and NSSI remains largely conceptual. 
Preliminary evidence for this bidirectional relationship between self-injury and emotional wellbeing comes from Lundh, Wångby-Lundh and Bjärehed (2011) who assessed the development of DSH and psychological problems (comprised of emotional problems, conduct problems, peer problems, and hyperactivity-inattention) in a sample of Swedish adolescents over two years. Psychological problems were found to prospectively predict new cases of repeated self-harm one year later, suggesting that overall psychological problems may be a risk factor for the onset of self-injury. In girls, self-harm prospectively predicted new cases of high risk psychological problems, suggesting a bidirectional relationship between risk factors. However, this reciprocal relationship was not observed in boys, leading Lundh and colleagues (2011) to speculate that self-harm may play a different role in the development of psychopathology among girls compared to boys. It is important to note that although this study included emotional problems among the composite of psychological problems, these items largely assessed depression-like symptoms rather than emotion dysregulation.

\section{The present studies}

At present, the specific nature of the relationship between emotion regulation and NSSI remains unclear. Initial research (e.g., Andrews et al., 2014; Whitlock et al., 2015) suggests that poor emotion regulation may be a risk factor for subsequent NSSI, but the potential reciprocal relationship has not been tested. This thesis aims to addresses these gaps in understanding. Study One tests the psychometric properties of two self-report measures of emotion regulation in an adolescent sample in order to inform the longitudinal research presented in Study Two. Study Two tests the proposed bidirectional relationship between emotion regulation and NSSI across a three year period in a cohort of community young adolescents. Study Three tests potential mediators of the relationship between NSSI and subsequent emotion regulation.

\section{Study One}

\section{Measuring self-reported emotion regulation in adolescents}

There are numerous self-report instruments designed to assess emotion regulation, some focusing on specific processes (Emotion Regulation Questionnaire; Gross \& John, 2003), as well as global emotion regulation ability (Cognitive Emotion Regulation Questionnaire: Garnefski, Kraaij, \& Spinnhoven, 2001; the Difficulties in Emotion Regulation Scale: Gratz \& Roemer, 2004; and the Regulation of Emotions Questionnaire 
Phillips \& Power, 2007). However, relatively few of these self-report measures were initially developed for use with adolescent populations. Although the Difficulties in Emotion Regulation Scale (DERS; Gratz \& Roemer, 2004) is commonly used in NSSI research, its length (36 items) makes it ill-suited for in-school assessment. Therefore, Study One aimed to validate the Emotion Regulation Index for Children and Adolescents (ERICA; MacDermott, Gullone, Allen, King, \& Tonge, 2010) self-report measure used in Study Two against the more widely used DERS, within a sample of New Zealand adolescents. ${ }^{1}$

The majority of the literature reviewed in the General Introduction assessed the extent of global dysfunctional emotion regulation using the DERS (see for example, Eichen et al., 2015; Gratz et al., 2012; Muehlenkamp et al., 2010; Whitlock et al., 2015; Yurkowski et al., 2015). The DERS is a 36-item self-report scale which draws directly from the Difficulties in Emotion Regulation Model and was initially developed in adult samples in order to assess clinically relevant difficulties in emotion regulation across six domains; Non-Acceptance of Emotional Responses (Non-Acceptance), Limited Access to Emotion Regulation Strategies (Strategies), Difficulties Engaging in Goal Directed Behaviour (Goals), Lack of Emotional Awareness (Awareness), Impulse Control Difficulties (Impulse), and Lack of Emotional Clarity (Clarity). Items are totalled to give an overall score of emotion dysregulation. Although women appear to score higher on the Goals, Strategies and Clarity subscales, there are typically no gender differences in overall DERS score, or on the Nonacceptance, Impulse or Awareness subscales (Bunford, Evans, \& Langberg, 2014; Weinberg \& Klonsky, 2009). Although developed with adult samples, the DERS has been shown to demonstrate good internal reliability, test-retest reliability, construct validity, and consistent factor structure within adolescent samples (Adrian, Zeman, Erdley, Lisa, \& Sim, 2011; Perez et al., 2012; Weinberg \& Klonsky, 2009). Additionally, individual differences in DERS has been shown to correspond with individual differences in respiratory sinus arrhythmia, a widely used physiological measure of emotion dysregulation (Vasilev, Crowell, Beauchaine, Mead, \& Gatzke-Kopp, 2009).

In contrast, the ERICA is 16-item self-report measure of emotion regulation competencies specifically developed for use with adolescents (MacDermott et al., 2010). The ERICA is comprised of three factors; Emotional Control, Emotional Self-Awareness and

\footnotetext{
${ }^{1}$ Note that the Youth Wellbeing Study longitudinal survey, from which Study Two and Study Three are drawn, was designed and Time 1 and 2 data collected prior to Study One. However in order to aid clarity the psychometric test of the ERICA (Study One) is presented first.
} 
Situational Responsiveness. Items are totalled to give an overall score of emotion regulation competencies. Gender differences in the ERICA have been largely unexplored, although Bunford and colleagues (2014) report a trend that girls may score higher than boys on the Self-Awareness subscale (although see Bunford, Evans, \& Wymbs, 2015). Across a range of child and adolescent samples, the ERICA has been shown to demonstrate reasonable internal consistency ( $\alpha$ 's range from .81 to .75), construct validity, and test-retest reliability (Bunford et al., 2014; Herzog, Hill-Chapman, Hardy, Wrighten, \& El-Khabbaz, 2015; MacDermott et al., 2010; Smith et al., 2016). Specifically in regards to emotion regulation strategies, the ERICA shows moderate positive correlations with self-reported cognitive reappraisal and small to moderate negative correlations with self-reported emotional suppression (Hughes, Gullone, \& Watson, 2011). Although the ERICA has previously been used in broader adolescent wellbeing research (see for example, Moreno, Garcia-Moya, Rivera, \& Ramos, 2016), the measure has not yet been used in NSSI research.

Very little research has directly compared the DERS and the ERICA within the same sample. Although not the focus of their work, Bunford and colleagues (2014) were the first to assess the relationship between the DERS and ERICA using a sample of adolescents with Attention-Deficit/Hyperactivity Disorder. Given that the DERS assesses emotion dysregulation and the ERICA assesses emotion regulation, conceptually we expect a strong negative relationship between the two scales, as well as the six DERS subscales and the three ERICA factors. Evidence for an association between ERICA and DERS was mixed, with approximately half of these correlations showing small to moderate negative associations (ranging from $r=$. -.15 to -.47) whereas the other half were non-significant. In particular, the ERICA Situational Awareness factor only correlated with the Clarity DERS subscale, suggesting that ERICA Situational Awareness is largely unrelated to DERS subscales. Given the moderate sample size $(N=180)$, these null effects are unlikely to be a result of Type II error. In subsequent analysis with a similar sample, overall ERICA and DERS scores were found to be modestly associated ( $r=-.26$; Bunford, Evans, Becker, \& Langberg, 2015). This pattern suggests that the ERICA and the DERS may not be as strongly associated as expected if they assess the same underlying construct. However, further research with a general adolescent community sample is needed.

An additional method of assessing the validity of the ERICA and the DERS is to assess the extent to which they demonstrate similar (but inverse) patterns of associations with other measures of psychological wellbeing. As the DERS is the most common tool for 
assessing emotion regulation in NSSI samples, it is critical that the ERICA shows a similar association with NSSI. In addition to NSSI, emotion regulation is also associated with other aspects of psychological wellbeing. Higher DERS scores have been found to be associated with greater depression (Bunford, Evans, \& Wymbs, 2015) and anxiety (Park, Edmondson, \& Lee, 2012), as well as poorer self-esteem (Garofalo, Holden, Zeigler-Hill, \& Velotti, 2016). Similarly, ERICA scores have been found to be associated with greater depression, although more weakly than has been demonstrated with DERS scores ( $r=-.22$ vs .57 , respectively; Bunford, Evans, \& Wymbs, 2015). However the association between ERICA scores and other aspects of psychological wellbeing has not yet been tested, highlighting the need for more research.

Study One aims to directly assess the relationship between the DERS and the ERICA in order to test the validity of the ERICA for use in Study Two and Three. The DERS and the ERICA were administered to a large sample of New Zealand adolescents. The factor structure and psychometric properties of each scale are examined and convergent validity assessed through associations with one another, as well as measures of depression, anxiety, and selfesteem. NSSI group differences in ERICA and DERS scores are examined, as well as associations with NSSI severity. Finally, multiple regression is used to assess whether DERS scores and ERICA scores uniquely predict NSSI severity. Given that the ERICA assesses emotion regulation competencies and the DERS assess emotion dysregulation, we expect a strong negative association between the two and inverse, but complimentary, associations with psychological wellbeing and NSSI measures.

\section{Method}

\section{Participants}

Participants were drawn from the Youth Wellbeing Study cross-sectional survey of 647 high school students enrolled in a co-educational, decile 8, secondary school in the greater Wellington region of New Zealand. Fifty-five percent $(N=353)$ of participants identified as female, $44 \%(N=285)$ as male, $0.5 \%$ as transgender ${ }^{2}$, and $0.6 \%$ did not indicate their gender. Participants ranged in age from 13 to 18 years old $(M=15.63, S D=1.38)$. The majority of participants (90.2\%) identified as New Zealand European/ Pākehā, 2.5\% as Māori

\footnotetext{
${ }^{2}$ As the number of adolescents who identified as transgender in the sample is too small to draw informative statistical conclusions, subsequent gender analyses will compare only participants who identify either as male or female.
} 
(indigenous New Zealanders), $0.6 \%$ as Chinese, $0.3 \%$ as Tongan, $4.7 \%$ as a non-listed ethnicity (e.g. South African, or Thai), and $1.7 \%$ could not choose a primary ethnicity.

\section{Materials}

\section{Emotion regulation}

Participants completed the 36-item Difficulties in Emotion Regulation Scale (DERS; Gratz \& Roemer, 2004) reviewed above (see pp. 26-27). Participants indicate how often items such as “When I'm upset, I believe that I will remain that way for a long time” apply to them on a 5 point scale where 1 is almost never (0-10\%) and 5 is almost always (91-100\%). The DERS is comprised of six subscales: Non-Acceptance of Emotional Responses, Difficulties Engaging in Goal Directed Behaviour, Impulse Control Difficulties, Lack of Emotional Awareness, Limited Access to Emotion Regulation Strategies, and Emotional Clarity. See Appendix A for the full measure. Eleven contrait items were reverse-coded (for example, "When I am upset, I feel like I can remain in control of my behaviours") before item scores were totalled to give subscale scores and an overall score of emotion dysregulation. Research providing psychometric validation of the DERS in adolescent samples is reviewed in the introduction to Study One. Within the current sample the DERS showed high internal consistency, $\alpha=.93$, and adequate inter-item correlations (Mean $r=$ $.30)$.

Participants also completed the 16-item ERICA (MacDermott et al., 2010) reviewed above (see pp. 27-28). Participants respond to statements such as "I handle it well when things change or I have to try something new" using a 5-point Likert scale ranging from 1 strongly disagree to 5 - strongly agree. The ERICA is comprised of three subscales: Emotional Control, Emotional Self-Awareness, and Situational Responsiveness. See Appendix B for the full measure. Ten contrait items were reverse-coded prior to analysis (for example, “When things don't go my way I get upset easily”). Item scores were then totalled to provide subscale and overall emotion regulation scores. Research providing psychometric validation of the ERICA is reviewed in the introduction to Study One. Within the current sample, inspection of the item-total correlations revealed that the item "When others are upset, I become sad or concerned for them" did not correlate well with the other items, $r=-$ .07. This item was removed from subsequent analyses (see Giles, 2013). The 15-item ERICA demonstrated excellent internal consistency, $\alpha=0.82$. 


\section{Non-suicidal self-injury}

All participants completed a screening question assessing NSSI history (see Appendix C). Participants who indicated they had engaged in NSSI, or thought about doing so, were then instructed to complete a modified version of Lundh, Karim and Quilisch's (2007) simplified version of Deliberate Self-Harm Inventory (DSHI-s). The DSHI-s was modified so the items "punched oneself" and "banged head" were combined into one (modified item "punched yourself, or banged your head against something, to the extent that caused a bruise to appear"). An additional scale point was also included differentiating "Never" into "I have never thought about doing this" and "I have thought about doing this, but never done it" in order to allow greater nuance in responding, allowing for closer analysis of the trajectory of NSSI behaviours and consistent with research arguing that thoughts about self-injury and engaging in self-injury are closely related, but often distinct (Martin, Bureau, Cloutier \& Lafontaine, 2011; Nock, Prinstein, \& Sterba, 2009). The revised DSHI-s consisted of $13-$ items containing descriptions of common forms of NSSI. Participants were asked to indicate the extent to which they had ever thought or engaged in each of the NSSI behaviours on a 5 point scale, ranging from 0 - "I've never thought about doing this" to 4 - "I've done this many times". Items began with "Have you ever deliberately (but without wanting to kill yourself)", and then listed 13 different self-injurious behaviours such as "cut your wrist, arms, or other areas of your body”. See Appendix D for the full measure. Consistent with previous research (see for example, Di Pierro, Sarno, Perego, Gallucci, \& Madeddu, 2012), participants' responses to the 13 items were averaged to provide an index of NSSI severity. Participants who indicated on the NSSI screening question that they had never thought about or engaged in NSSI were assigned a DSHI-s score of zero. The DSHI has previously demonstrated good internal consistency $(\alpha=.82$, Gratz, 2001) and the simplified version has been validated for use in adolescent samples (Lundh et al., 2007). Within the current sample, the DSHI-s showed excellent internal consistency, $\alpha=0.86$.

Participants who reported a lifetime history of NSSI also completed the Inventory of Statement About Self-Injury (ISAS; Klonsky \& Glenn, 2009) to assess the functions their self-injury serves. The ISAS is a self-report measure which begins with "When I self-injure, I am" followed by 39 items describing functions of NSSI such as "releasing emotional pressure that has built up inside of me". Participants indicate how relevant each item is to their self-injury, responding on a 3-point Likert scale ranging from ' 0 - not relevant' to '2 very relevant'. Three items each are averaged to create scores for Affect Regulation, 
Interpersonal Boundaries, Self-Punishment, Self-Care, Anti-Dissociation/Feeling Generation, Anti-Suicide, Sensation-Seeking, Peer-Bonding, Interpersonal Influence, Toughness, Marking Distress, Revenge, and Autonomy functions, which are grouped into two superordinate clusters of Intrapersonal functions and Interpersonal functions (see pp. 19-20 for further description). The full measure is presented in Appendix E. The ISAS has previously demonstrated good internal consistency $(\alpha=.85$ for the Interpersonal Functions superordinate factor, and $\alpha=.80$ for the Intrapersonal Functions factor; Klonsky \& Glenn, 2009) and has been validated for use in adolescent sample (Kortge et al., 2013). Within the current sample, both the Intrapersonal and Interpersonal subscales showed adequate internal consistency, $\alpha=78$ for both subscales. The ISAS was administered primarily to assess the representativeness of the NSSI sample compared to international samples.

\section{Depression and Anxiety}

Depression and anxiety were measured using the relevant 7 -item subscales of the Depression, Anxiety and Stress Scale (DASS-21; Henry \& Crawford, 2005). Participants reported to items such as "I couldn't seem to experience any positive feeling at all' using a 4point Likert scale where 0 is did not apply to me all and 3 is applied to me very much, or most of the time. See Appendix F for the full measure. All seven items in each subscale were totalled, with higher scores indicating greater depression or anxiety. Previous research has found both the depression and anxiety subscales of the DASS-21 demonstrate adequate internal consistency and construct validity within young adult and adolescent samples (Norton, 2007; Szabó, 2010). In the current sample, both the depression and anxiety subscales showed good internal consistency ( $\alpha=89$ and .86 , respectively).

\section{Self-Esteem}

Self-esteem was indexed using the 10 item Rosenberg Self-Esteem Scale (RSES, Rosenberg, 1965). Participants respond to items such as "Overall, I am satisfied with myself" using a 4-point Likert scale ranging from 1 - strongly disagree to 4 - strongly agree. Five items were reverse coded (for example, "I feel I do not have much to be proud of") and item scores totalled to provide an overall self-esteem score with higher scores indicating higher self-esteem. See Appendix G for the full measure. Previous research has found the RSES to demonstrate excellent internal reliability and be validate for use in adolescent samples (Bagley \& Mallick, 2001; Vispoel, Boo, \& Bleiler, 2001). The RSES showed excellent internal consistency $(\alpha=.92)$ in the current sample. 


\section{Procedure}

Ethical approval for this study was granted by the National Health and Disability Ethics Committee (MEC/11/12/108). The secondary school was contacted and invited to take part in the survey in early 2015. All students enrolled at the school were eligible to take part. Students aged 16 or over gave their own consent to participate in the survey, while opt-in parental consent was required for students aged under 16 (as at $15^{\text {th }}$ June, 2015). Students aged under 16 took home an information pack and a consent form for parents. In order to maximize the return rate, students who returned a completed consent form (regardless of whether parents consented or not) were given a small chocolate as thanks. Three hundred and sixty-nine completed consent forms were returned, with $82.4 \%$ providing consent for the student to take part in the study. This overall rate of consent is consistent with other longitudinal high school-based NSSI research requiring active parental consent (e.g., Andrews et al., 2014). For students aged under 16, both parental consent and individual assent was required prior to participation.

The data collection was conducted over four days in June of 2015. The survey was administered in classrooms during class periods under the supervision of a research team member. Participants were first given an information sheet outlining the study, a verbal briefing, and the opportunity to ask researchers any questions. Participants were informed that their participation in the current study was voluntary and that they were able to withdraw at any time without consequence. Participants were also given assurance that their answers would remain confidential. The survey consisted of a series of questionnaire measures assessing youth wellbeing (additional to those pertinent to this study), and took approximately 40 minutes to complete. In general, demographic questions were presented first, followed by NSSI measures and then other psychological wellbeing measures. Following participation, students were debriefed and given contact details for community services, should they wish to seek support. Students were also able to approach team members following participation if they had any questions or concerns. Students also had the option of leaving their email or postal address to receive a summary of study results.

In order to maximise the sample size available for all analyses, except for the Principal Components Analyses, missing data was computed where possible. Given that the Depression subscale, the Anxiety subscale, the RSES, the DERS and the ERICA all show adequate internal consistency, when participants had completed $80 \%$ or more of the scale the average of the items they had completed was calculated and inserted into the missing cells 
before a sum score was calculated. In contrast, the DSHI-s is more like a symptoms check list than a typical scale. Therefore, when a participant had not completed all items of the DSHI-s, missing cells were assumed to be the absence of that NSSI behaviour and replaced with zeros (see Lundh, Karim \& Qulisch, 2007 for a similar method of addressing missing DSHI-s data).

\section{Results and Discussion}

\section{DERS Principle Component Analysis}

An exploratory Principal Components Analysis (PCA) with Varimax orthogonal rotation and Kaiser normalization was conducted to determine the factor structure of the full 36 -item DERS in an adolescent sample. Both Bartlett's Test of Sphericity $\chi^{2}(630)=$ $12603.45, p<.001$ and Keyser-Meyer-Olkin test of .94 indicated that the data was suitable for PCA.

Inspection of the eigenvalues and scree plot yielded mixed results (see Figure 5). Eight components with eigenvalues greater than one (Kaiser, 1974) accounted for $70.00 \%$ of the variance. However, inspection of the scree plot suggested a two or three factor solution. Given that Kaiser's criterion has often been critiqued for resulting in over-extraction (Velicer, Eaton, \& Fava, 2000) and the ambiguity of the current scree plot, Horn's (1965) parallel analysis was conducted to help determine the appropriate number of factors to retain. Parallel analysis compares the eigenvalues produced from the sample data set with those from a randomly generated data set. Velicer and colleagues (2000) argue that by retaining only components which have larger eigenvalues than those generated randomly, parallel analysis is more accurate than either Kaiser's criterion or the use of a Catell's scree plot. Using a randomly generated data set, eigenvalues of 1000 correlation matrices were calculated. For each component, the eigenvalue representing the $95 \%$ percentile of generated eigenvalues was taken as the critical value. The first five PCA components were above the respective critical values generated by the parallel analysis and so were retained. However, the sixth, seventh and eighth PCA components resulted in the eigenvalues exceeding those produced from a random data set at the $95^{\text {th }}$ percentile, and so were rejected (see Table 1). Therefore, the more stringent method of parallel analysis suggested a five component structure was most appropriate within this data set. 


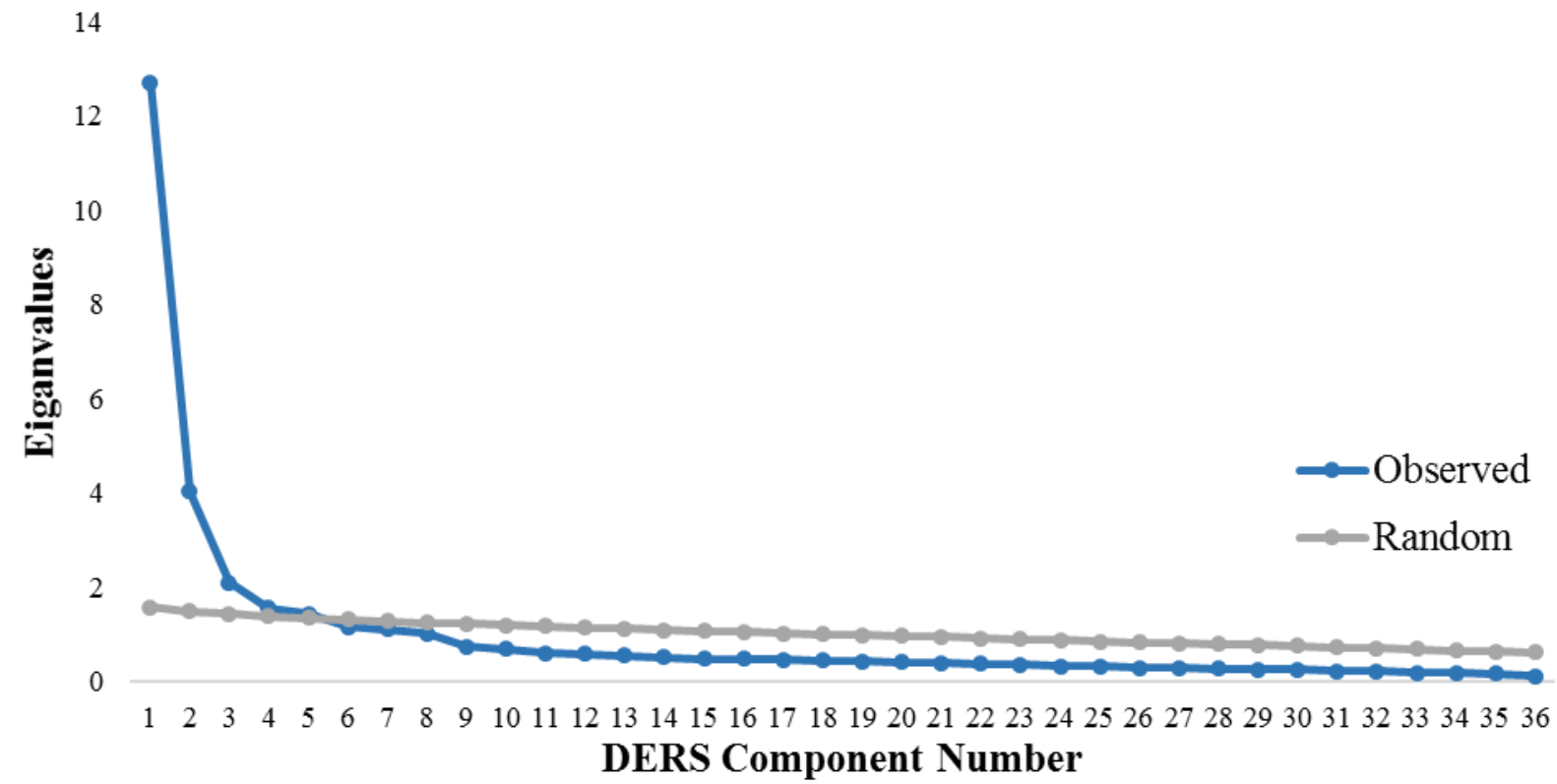

Figure 5. Scree plot displaying both the observed eigenvalues generated from the initial Principal Components Analysis and the random eigenvalues generated by the parallel analysis, for each of the 36 possible Difficulties in Emotion Regulation Scale components in Study One.

Table 1

Results of Parallel Analysis used to determine the most appropriate number of components within the Difficulties in Emotion Regulation Scale in Study One

\begin{tabular}{cccc}
\hline Component & PCA Eigenvalue & $\begin{array}{c}\text { Parallel Analysis Criterion } \\
\text { Value }\end{array}$ & Result \\
\hline 1 & 12.71 & 1.58 & Accept \\
2 & 4.04 & 1.50 & Accept \\
3 & 2.11 & 1.45 & Accept \\
4 & 1.57 & 1.40 & Accept \\
5 & 1.45 & 1.36 & Accept \\
6 & 1.17 & 1.32 & Reject \\
7 & 1.12 & 1.29 & Reject \\
8 & 1.03 & 1.26 & Reject
\end{tabular}

Note. Sample $N=553$, Generated data set $N=1000$ 
Table 2

Items comprising the Difficulties in Emotion Regulation Scale with factor loadings in Study One.

\begin{tabular}{|c|c|c|c|c|c|}
\hline \multirow[t]{2}{*}{ Items } & Factor 1 & Factor 2 & Factor 3 & Factor 4 & Factor 5 \\
\hline & Non-Acceptance & Goals & Awareness & Impulse & Clarity \\
\hline
\end{tabular}

When I'm upset, I feel ashamed with myself for feeling that way.

.76

.68

.68

When I'm upset, I believe that I will remain that way for a long time.

.68

.68

When I'm upset, I feel like I am weak.

When I'm upset, I become irritated with myself.

.66

.64

.63

.58

.58

.50

.48

.49

When I'm upset, my emotions feel overwhelming.

.49

When I'm upset, I have difficulty focusing on other things.

When I'm upset, I have difficulty getting work done. 
When I'm upset, I can still get things done (R).

When I'm upset, I acknowledge my emotions (R).

.76

.75

I am attentive to my feelings ( $R$ ).

care about what I am feeling $(\mathrm{R})$

I pay attention to how I feel (R).

When I'm upset, I believe my feelings are valid and important (R).

When I'm upset, I take time to figure out what I'm really feeling (R).

I am clear about my feelings (R).

When I'm upset, I lose control over my behaviours.

When I'm upset, I become out of control.

When I'm upset, I have difficulty controlling my behaviours

When I'm upset, I feel out of control.

I experience my emotions as overwhelming and out of control.

I have difficulty making sense out of my feelings.

I have no idea how I'm feeling.

I'm confused about what I'm feeling

I know exactly how I am feeling (R).

.49

Note. Items loading on each factor are bolded, Factor loadings below .30 are not presented for ease of interpretation. 
A second PCA was conducted, limiting the number of components to five. Item loadings are presented in Table 2. The five factor solution explained $60.76 \%$ of the variance. This factor structure is moderately different to that originally reported by Gratz and Roemer (2004). The first factor, explaining $35.30 \%$ of the variance and made up of 13 items, is a composite of the two factors originally labelled Non-Acceptance and Strategies except for the addition of two items ("I am clear about my feelings (R)") and "When I'm upset, I know that I can find a way to eventually feel better (R)"). The fourth factor, explaining $4.36 \%$ of the variance and made up of 6 items, is identical to the factor originally labelled Impulse Control Difficulties (Impulse). The fifth factor, explaining 4.02\% of the variance and made up of 4 items, mirrors the factor originally labelled Lack of Emotional Clarity (Clarity) except that it excludes one item ("I am clear about my feelings (R)").

Taken together, this PCA analysis suggests that within this adolescent sample, the DERS is best described using a five factor structure in which Nonacceptance and Strategies comprise a single factor, and the Goals, Impulse, Awareness, and Clarity factors largely mirror the original factor structure reported by Gratz and Roemer (2004). Although the original DERS factor structure demonstrated in adults has been replicated in adolescents aged 13-17 years old (Weinberg \& Klonsky, 2009), the current psychometric study suggests that adolescent responses may not systematically differentiate between the Non-Acceptance and Strategies subscales.

However, the PCA provides evidence for substantial cross-loading for some items, particularly the items “When I'm upset, it takes me a long time to feel better", "When I'm upset, I believe that wallowing in it is all I can do", and "When I'm upset, my emotions feel overwhelming”. Notably, all three of these items were originally reported by Gratz and Roemer (2004) to cluster in the Strategies factor, so perhaps if the PCA was not restricted to five factors (compared to the original six factor structure) this cross-loading would be reduced. However, a five factor solution was retained due to the more stringent results of the parallel analysis. In order to maintain consistency within the literature, the five factors will be referred to as Nonacceptance and Strategies, Goals, Impulse, Awareness, and Clarity.

Comparable subscale scores were created by averaging across the items in each scale. The Nonacceptance and Strategies $(\alpha=.91)$, Goals $(\alpha=.88)$, Awareness $(\alpha=.83)$, Impulse $(\alpha=.87)$, and Clarity ( $\alpha=.81)$ subscales all showed good internal consistency. The majority of the DERS subscales showed large positive associations with each other ( $r$ s range from .41 
Table 3

Descriptive statistics for the Difficulties in Emotion Regulation Scale total and subscale scores split by gender in Study One

\begin{tabular}{lccccc}
\hline \multirow{2}{*}{ Variable } & \multicolumn{2}{c}{ Female } & & \multicolumn{2}{c}{ Male } \\
\cline { 2 - 3 } \cline { 4 - 6 } & $M$ & $(S D)$ & & $M$ & $(S D)$ \\
\hline 1. DERS Total & $86.57^{*}$ & $(25.72)$ & & $77.95^{*}$ & $(20.79)$ \\
2. Nonacceptance and Strategies & $2.22^{*}$ & $(1.01)$ & & $1.80^{*}$ & $(0.80)$ \\
3. Goals & $2.90^{\wedge}$ & $(1.06)$ & & $2.72^{\wedge}$ & $(1.01)$ \\
4. Impulse & $1.92^{\wedge}$ & $(0.84)$ & & $1.75^{\wedge}$ & $(0.78)$ \\
5. Awareness & 2.78 & $(0.76)$ & & 2.78 & $(0.75)$ \\
6. Clarity & $2.39^{*}$ & $(0.86)$ & & $2.08^{*}$ & $(0.82)$ \\
\hline
\end{tabular}

Note. ${ }^{*} p<.001 .^{\wedge} p$ s range from .034 to .001

to .65, see Table 6). Similar to previous research with adult and adolescent samples (Bunford et al., 2014; Gratz \& Roemer, 2004), the Awareness subscale showed systematically weaker associations with the other subscales ( $r$ s range from .09 to .21 ).

In order to examine for potential gender differences, a 2 x 5 repeated measures Analysis of Variance (ANOVA) was conducted with one between-subjects factor (Gender; Male, Female) and one within-subjects factor (DERS; Nonacceptance and Strategies, Goals, Impulse, Awareness, Clarity). Mauchley's test of Sphericity indicated that the assumption of sphericity had been violated, $\chi^{2}(9)=2.17 .90, p<.001$. With a Greenhouse-Giesser correction applied, a main effect of DERS subscale was found, $F(4,1759.83)=230.25, p<.001, \eta p^{2}=$ .30 , as well as a main effect of gender, $F(4,546)=15.46, p<.001, \eta \mathrm{p}^{2}=.03$. These main effects are qualified by an interaction between DERS and gender, $F(3.22,1759.83)=8.16$, $p$ $<.001, \eta \mathrm{p}^{2}=.02$. Follow up between subjects $t$ tests revealed that girls scored higher than boys on the Nonacceptance and Strategies, Goals, Impulse, and Clarity subscales, but no different on the Awareness subscale (see Table 3 for details). Given that in this study the Nonacceptance and Strategies subscales were combined, this pattern of results is similar to the gender differences previously reported in an adolescent sample (Weinberg \& Klonsky, 2009), with the exception of the Impulse subscale. 
Overall, within this New Zealand community adolescent sample the DERS shows good internal reliability and moderate deviations from the factor structure, as well gender differences similar to those previously reported within adolescent samples (Weinberg \& Klonsky, 2009).

\section{ERICA Principle Component Analysis}

An exploratory Principle Components Analysis (PCA) with Varimax orthogonal rotation and Kaiser normalization was conducted with the 15 ERICA items. Both Bartlett's Test of Sphericity $\chi^{2}(105)=2611.17, p<.001$ and Keyser-Meyer-Olkin test of .83 indicated that the data was suitable for PCA. Three components with eigenvalues of greater than one (Kaiser, 1974) accounted for $51.11 \%$ of the variation. However, inspection of Cattell's (1966) scree plot suggested either a three or four component solution (see Figure 6). Again, Horn's (1965) parallel analysis was conducted to help determine the appropriate number of factors to retain.

Using a randomly generated data set, eigenvalues of 1000 correlation matrices were calculated. For each component, the eigenvalue representing the $95 \%$ percentile generated eigenvalues was taken as the critical value (see Table 4). The first three PCA components eigenvalues were above the respective critical values generated by the parallel analysis and so were accepted. However, the fourth PCA component provided an eigenvalue of 0.98 , below the 1.16 cut off recommended by parallel analysis and so was rejected. As a result, a second PCA was conducted, this time limiting the number of components to three.

Consistent with the factor structure reported by MacDermott and colleagues (2010), the final PCA three components explained $51.11 \%$ of the variance in the data. Item loadings are presented in Table 5. Two items, "When things don't go my way I get upset easily" and "I enjoy seeing others hurt or upset", showed some evidence of cross loading across factors. Item loadings broadly replicated the loading pattern originally reported by MacDermott and colleagues (2010). The first component, originally labelled Emotion Control, explained $29.84 \%$ of the variance and was comprised of all seven of the original items, plus an additional item originally from the third component (Situational Responsiveness). The second component, labelled Emotional Self-Awareness, explained $12.57 \%$ of the variance and mirrored the original five items. The final component, labelled Situational Responsiveness, explained $8.70 \%$ of the variance. This Situational Responsiveness factor showed the most change from the original structure in that one item ("When others are upset, I become sad or 


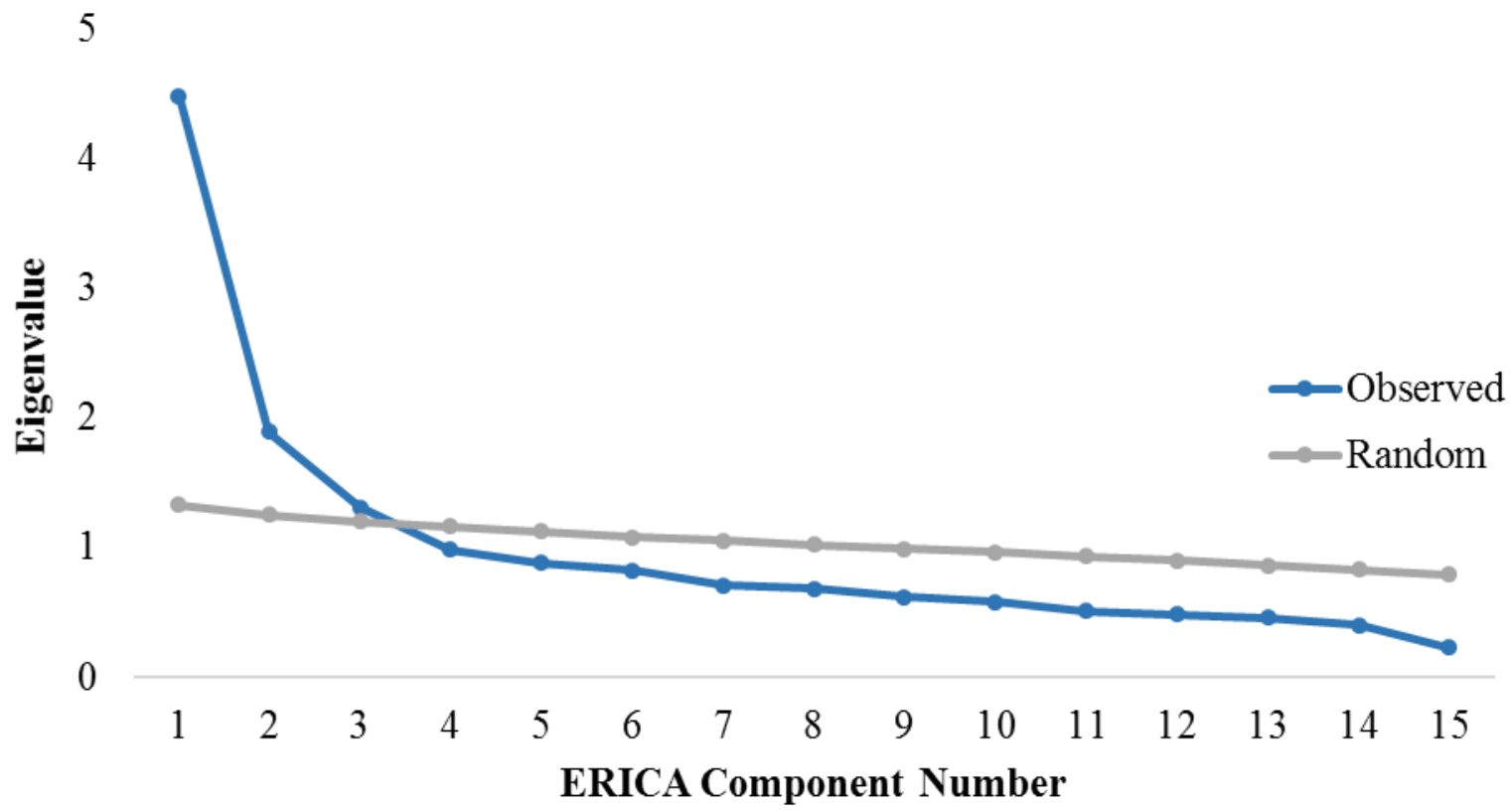

Figure 6. Scree plot displaying the observed eigenvalues generated from the initial Principal Components Analysis as well as the random eigenvalues generated from the parallel analysis, for each of the possible 15 components of the Emotion Regulation Index for Children and Adolescents in Study One.

Table 4

Results of Parallel Analysis used to determine the most appropriate number of components within the Emotion Regulation Index for Children and Adolescents in Study One.

\begin{tabular}{cccc}
\hline Component & PCA Eigenvalue & $\begin{array}{c}\text { Parallel Analysis Criterion } \\
\text { Value }\end{array}$ & Result \\
\hline 1 & 4.48 & 1.33 & Accept \\
2 & 1.89 & 1.25 & Accept \\
3 & 1.31 & 1.20 & Accept \\
4 & 0.98 & 1.16 & Reject
\end{tabular}

Note. Sample $N=613$, Generated 1000 correlation matrices. 
Table 5

Items comprising the Emotion Regulation Index for Children and Adolescents with factor loadings in Study One.

\begin{tabular}{|c|c|c|c|}
\hline Items & $\begin{array}{l}\text { Factor } 1 \\
\text { Emotional Control }\end{array}$ & $\begin{array}{c}\text { Factor } 2 \\
\text { Emotional } \\
\text { Self-Awareness }\end{array}$ & $\begin{array}{c}\text { Factor } 3 \\
\text { Situational } \\
\text { Responsiveness }\end{array}$ \\
\hline I am a happy person. & & .79 & .33 \\
\hline I handle it well when things change or I have to try something new. & & .65 & \\
\hline When I get upset, I can get over it quickly. & & .76 & \\
\hline I am a sad person $(\mathrm{R})$. & & .77 & \\
\hline I am quiet and shy, and I don't show my feelings (R). & & .52 & \\
\hline When other kids are friendly to me, I am friendly to them. & & & .83 \\
\hline When adults are friendly to me, I am friendly to them. & & & .76 \\
\hline When things don't go my way I get upset easily (R). & .51 & .38 & \\
\hline I have angry outbursts (R). & .60 & .38 & \\
\hline I enjoy seeing others hurt or upset (R). & .34 & & \\
\hline I can be disruptive at the wrong times $(\mathrm{R})$. & .72 & & \\
\hline I get angry when adults tell me what I can and cannot do (R). & .62 & & \\
\hline I have trouble waiting for something I want $(\mathrm{R})$. & .65 & & \\
\hline I do things without thinking about them first (R). & .67 & & \\
\hline I annoy others by not minding my business (R). & .66 & & \\
\hline
\end{tabular}

Note. Items loading on each factor are bolded, Factor loadings below .30 are not presented for ease of interpretation. 
concerned for them") was removed because it did not correlate well with the other items on the scale and one item ("I enjoy seeing others hurt or upset") showed marginally higher loading on Emotional Control. As a result, in the present study Situational Responsiveness is only comprised of two items.

Both the Emotional Control and the Emotional Self-Awareness subscales showed adequate internal consistency (both $\alpha=.77$ ). The reliability coefficient for Situational Responsiveness ( $\alpha=.68$ ) was lower than traditionally acceptable (Nunnally, 1978), although inspection of the inter-item correlation demonstrated that the two items were strongly correlated $(r(643)=.52)$. The scores for each factor were then summed and averaged to create comparable subscale scores. Bivariate correlations were conducted and small to moderate positive associations were found between all three subscales ( $r$ s ranging from .26 to .39, see Table 6), suggesting that while these three factors exhibit a degree of overlap they may also be conceptualised as distinct factors.

In order to examine gender differences, a repeated measures Analysis of Variance (ANOVA) with one between-subject factor (Gender; male, female) and one within-subjects factor (ERICA; Emotional Control, Emotional Self-Awareness, and Situational Responsiveness) was conducted. No evidence was found for a main effect of gender, $F(1$, $605)=2.28, p=.132, \eta p^{2}<.01$. There was for a main effect of ERICA subscale, $F(2,1210)$ $=489.93, p<.001, \eta p^{2}=.45$. However, these main effects are qualified by an interaction between ERICA and gender, $F(2,1210)=35.99, p<.001, \eta p^{2}=.06$. Girls reported better Emotional Self-Awareness, and Situational Responsiveness than did boys (see Table 7 for details). The gender difference in Emotional Self-Awareness is consistent with a trend previously reported (Bunford et al., 2014).

Overall, within this sample of New Zealand community adolescents the ERICA shows good internal reliability and largely mirrored the factor structure originally reported (MacDermott et al., 2010). Girls reported greater Emotional Self-Awareness and Situational Responsiveness factors than did boys. 
Table 6

Correlations between the Emotion Regulation Index for Children and Adolescent and the Difficulties in Emotion Regulation Scale total scores and subscales in Study One

$\begin{array}{lllllllll}1 . & 2 . & 3 . & 4 . & 5 . & 6 . & 7 . & 8 . & 9 .\end{array}$

1. ERICA Emotion Regulation Total Score

2. DERS Emotion Dysregulation Total Score $\quad-.72^{*}$

3. ERICA Emotional Self-Awareness $\quad .78^{*} \quad .71^{*}$

4. ERICA Situational Responsiveness $\quad .49^{*} \quad-.26^{*} \quad .38^{*}$

5. ERICA Emotional Control $\quad .87^{*} \quad-.53^{*} \quad .39^{*} \quad .26^{*}$

6. DERS Nonacceptance and Strategies $\quad \begin{array}{llllll}-.58^{*} & .90^{*} & -.62^{*} & -.16^{*} & -.40^{*}\end{array}$

$\begin{array}{llllllll}\text { 7. DERS Goals } & -.52^{*} & .74^{*} & -.48^{*} & -.09^{\wedge} & -.44^{*} & .61^{*}\end{array}$

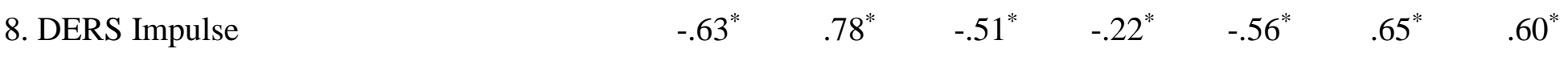

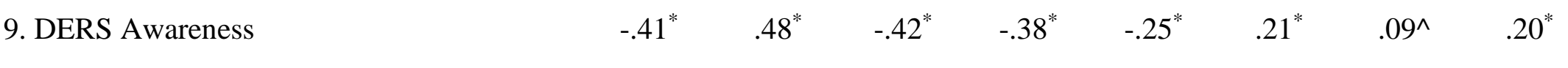

10. DERS Clarity $\quad-.54^{*} \quad \begin{array}{lllllllll} & .71^{*} & -.54^{*} & -.24^{*} & -.38^{*} & .54^{*} & .41^{*} & .48^{*} & .43^{*}\end{array}$

Note. $* p<.001, \wedge p$ s range from .05 to $.001 . N$ s range from 533 to 613. 
Table 7

Descriptive statistics for Emotion Regulation Index for Children and Adolescents total and

\begin{tabular}{lccccc}
\hline \multirow{2}{*}{ Variable } & \multicolumn{2}{c}{ Female } & & \multicolumn{2}{c}{ Male } \\
\cline { 2 - 3 } \cline { 5 - 6 } \cline { 5 - 6 } ERICA Total & $M$ & $(S D)$ & & $M$ & $(S D)$ \\
Emotional Control & 56.08 & $(7.89)$ & & 57.19 & $(7.63)$ \\
Emotional Self-Awareness & 3.67 & $(0.63)$ & & 3.63 & $(0.64)$ \\
Situational Responsiveness & $3.54^{*}$ & $(0.75)$ & & $3.88^{*}$ & $(0.67)$ \\
\hline
\end{tabular}

subscales scores, split by gender in Study One

Note. * ${ }^{*}<.001$.

Comparing across both scales, the DERS demonstrates more cross-loading of items than does the ERICA. This greater cross-loading may be tied to nature of the items within each scale. The ERICA was initially developed from the Emotion Regulation Checklist (ERC, Shields \& Cicchetti, 1997), an observational tool to be completed by adults familiar with the child being assessed. As such, the items tend to describe behavioural outcomes of failure to effectively regulate emotions (e.g., "I get angry when adults tell me what I can and cannot do (R)"). In contrast, the DERS assesses emotion regulation using a wider range of measurements including the behavioural consequences of poor emotion regulation in terms of goal pursual and poor impulse control (Goals and Impulse subscales, respectively), emotional awareness and clarity (Awareness and Clarity subscales), ineffective use of strategies (Strategies subscale) and acceptance of the emotional response (Non-Acceptance subscale). However, adolescents within the current sample did not distinguish between Strategies and Nonacceptance subscales in the same manner as previous adult and adolescent samples (Gratz \& Roemer, 2004; Weinberg \& Klonsky, 2009). This lack of distinction may reflect developmental changes in emotional awareness. Lane and Pollermann (2002) argue that emotional awareness develops progressively from early stages focused on recognition of emotion and the understanding of behavioural tendencies, to later stages involving awareness and understanding of complex blends of emotions (e.g., feeling angry because you are sad). These early stages of emotional awareness may be captured by the DERS Clarity, Awareness, 
Impulse, and Goal subscales, while the Strategies and Nonacceptance subscale capture the later stages of emotional awareness.

\section{Construct Validity}

Given that the ERICA and the DERS are measures designed to assess self-reported emotion regulation, participant's scores for each should be strongly correlated. Note that the ERICA is worded such that a higher score indicates greater emotion regulation ability, whereas the DERS is worded such that a higher score indicated greater emotion dysregulation. Indeed, the large correlation of $r(636)=-.72$ between ERICA and DERS provides evidence for high convergent validity. This association is considerably stronger than previously reported ( $r=-.26$; Bunford et al., 2015), perhaps due to the difference in samples (community adolescents vs. adolescents with Attention-Deficit/Hyperactivity Disorder). When separated into subscales, overall there were moderate to strong associations between the ERICA factors and the DERS factors (see Table 6). However at face value, ERICA Situational Responsiveness and the DERS Awareness factors do not show as strong associations as the other factors. This lack of convergent validity across emotion regulation self-reported measures is consistent with previous research (Zelkowitz \& Cole, 2016). Given that the overall ERICA and DERS scores share a strong association, and that some subscales were made up of a small number of items, subsequent analyses focuses exclusively on the total sum scores of the ERICA and the DERS.

Next, I tested to see if the ERICA and the DERS total scores were associated in a similar manner with self-reported depression $(M=3.66, S D=4.42)$, anxiety $(M=3.43, S D=$ 4.12), and self-esteem $(M=29.32, S D=6.05)$ (see Table 8). As predicted, greater DERS emotion dysregulation scores were associated with greater depression and anxiety, and lower self-esteem. Showing the expected inverse pattern, greater ERICA emotion regulation scores were associated with lower scores of depression and anxiety, and greater self-esteem. In order to compare the magnitude of the association between wellbeing and DERS and ERICA scores, the sign of the correlations was ignored and the dependent correlations were compared using online software (Lee \& Preacher, 2013). Controlling for the association between DERS and ERICA scores, DERS scores were found to be more strongly associated with depression $(Z=6.68, p<.001)$, anxiety $(Z=5.56, p<.001)$, and self-esteem $(Z=5.25, p$ $<.001)$ than were ERICA scores. This pattern of results suggests that although both ERICA and DERS are generally strongly associated with both positive and negative aspects of wellbeing (as per Cohen's 1960 guidelines), these associations are significantly stronger for 
Table 8

Zero-order correlations between the Emotion Regulation Index for Children and Adolescents, the Difficulties in Emotion Regulation Scale, Depression, Anxiety, and Self-Esteem in Study On

$\begin{array}{llll}1 . & 2 . & 3 .\end{array}$

1. ERICA Total Score

2. DERS Total Score

$-.72^{*}$

3. DASS Depression

$-. .57^{*}$ $.72^{*}$

4. DASS Anxiety

$-.46^{*}$

$.60^{*}$

$.65^{*}$

5. RSES Self-Esteem

$.65^{*}$

$-.75^{*}$

$-.67^{*}$

$-.49^{*}$

Note. $* p<.001$. Ns range from 615 to 638.

the DERS than for the ERICA. This disparity between the DERS and the ERICA is consistent with previous work (Bunford, Evans, Becker \& Langberg, 2015).

\section{Associations with NSSI status and NSSI severity}

Given that NSSI is a key focus of interest within this thesis, the relationship between NSSI and both ERICA emotion regulation and DERS emotion dysregulation was assessed. As expected in a community sample, the majority of participants $(71.1 \%)$ reported no history of self-injury. Similar to international prevalence rates (Muehlenkamp et al., 2012; Swannell, Martin, Page, Hasking, \& St John, 2014), 19.7\% of the current sample reported a life time history of NSSI. An additional 9.2\% had thought about engaging in NSSI. Girls were more likely to report having engaged in NSSI (28.2\%) than were boys (8.4\%), and were also more likely to report having thought about engaging in NSSI (12.9\%) than were boys $(4.4 \%), \chi^{2}{ }_{(2)}$, $=59.76, p<.001$, Cramer's $V=.31$. Consistent with international research (Klonsky \& Glenn, 2009; Whitlock et al., 2011), among participants who reported engaging in NSSI the most common behaviours were cutting, scratching, and punching or banging oneself, and affect regulation and self-punishment were the most highly endorsed functions (see Table A and B in Appendix H).

In order to test whether both the DERS and the ERICA replicate the well-established group difference in emotion regulation between people with NSSI and people without NSSI, 
three quasi-experimental groups were created based on participant's self-reported NSSI history. Participants who reported a life-time history of NSSI were assigned to the Engagement group $(N=122)$, participants who reported thinking about engaging in NSSI were assigned to the Thoughts of Engagement group $(N=56)$ and participants who had never engaged in NSSI or thought about engaging in NSSI were assigned to the No History group $(N=371)$. Note that as participants were recruited from a community sample, the groups were substantially different in size. Although previous research has compared people with and without NSSI, there has been less systematic analysis of people with thoughts about selfinjury. Thinking about engaging in NSSI may represent a critical step towards NSSI engagement (Martin et al., 2011). As such, these people are an important group to understand in terms of both theory and treatment.

A one-way MANOVA was conducted in order to compare DERS and ERICA total scores by NSSI status; No History $(N=440)$, Thoughts of Engagement $(N=57)$, and Engagement $(N=122)$. There was a statistically significant difference in emotion regulation based on NSSI status, $F(4,1218)=42.97, p<.001$; Wilk's $\Lambda=0.77, \eta p^{2}=.12$. A univariate test revealed that NSSI severity differed by both ERICA scores, $F(2,612)=59.03, p<.001$, $\eta p^{2}=.16$, and DERS scores, $F(2,612)=87.65, p<.001 \eta p^{2}=.22$. Consistent with previous research (Eichen et al., 2015; Muehlenkamp et al., 2010), the Engagement group ( $M=$ 100.63, $S D=25.98)$ reported higher DERS scores than did the No History group $(M=75.77$, $S D=19.85), p<.001, d=-1.07,95 \%$ CI $[-30.00,-19.71]$. The Thoughts of Engagement group $(M=101.58, S D=21.55)$ also reported greater DERS scores than did the No History group, $p<.001, d=-1.21,95 \%$ CI [-32.94, -18.69]. However, no difference was found between the Engaged and Thoughts of Engagement groups, $p=.959, d=0.02,95 \%$ CI [9.05, 97.15]. As predicted and consistent with the inverse of the pattern found for the DERS scores, follow-up planned comparisons revealed that participants who had Engaged in NSSI $(M=51.48, S D=7.70)$, reported lower ERICA scores compared to participants with No History of NSSI $(M=58.48, S D=7.18), p<.001, d=0.94,95 \%$ CI [5.27, 8.74]. Participants who reported Thoughts of Engagement $(M=51.79, S D=6.38)$ also reported lower ERICA scores than participants with No History, $p<.001, d=0.99,95 \%$ CI $[4.31,9.08]$. However, there was no difference between the Engaged and Thoughts of Engagement groups, $p=.961$, $d=0.04,95 \%$ CI $[-3.03,2.41]$.

Both the DERS and the ERICA replicated the well-established group difference between people with NSSI and people without NSSI. Exploratory analysis comparing people 
with thoughts of NSSI to people with and without NSSI revealed that people with thoughts of NSSI cannot be distinguished from people with NSSI by emotion regulation scores. Given the argument that for some people thoughts of NSSI lead to NSSI engagement (Martin et al., 2011), this pattern of results tentatively suggests that deficits in emotion regulation may precede NSSI engagement. Study Two will test this hypothesis directly.

Taking a more nuanced approach to include the heterogeneity within groups, the associations between NSSI severity and both DERS and ERICA scores were also tested. Given that NSSI severity (Mean $=0.19, S D=0.41$, Median $=0.00)$ was non-normally distributed, with skewness of $2.62(S E=.10)$ and kurtosis of $7.48(S E=0.20)$, nonparametric Spearman's correlations were conducted. Consistent with previous research, DERS scores were positively associated with NSSI severity, $r_{s}(597)=.46, p<.001$, and ERICA scores were negatively associated with NSSI severity, $r_{s}(600)=-.41, p<.001$. However, given the large correlation between DERS and ERICA, it is not clear from zeroorder correlations whether each scale is uniquely predicting NSSI severity. In order to test whether both the DERS and ERICA are independently associated with NSSI severity, a simultaneous inclusion regression was conducted where NSSI severity was regressed onto the DERS and ERICA. The overall regression was significant, $F(2,594)=88.27, p<.001$ and the DERS and ERICA combined were found to explain approximately $23 \%$ (Adjusted $R^{2}=.23$ ) of the variance in NSSI severity. As predicted, the DERS score was a moderate positive predictor of NSSI severity $(\beta=.41, p<.001)$. However, ERICA scores did not uniquely predict NSSI severity over and above the DERS $(\beta=-.09, p=.075)$. Examination of the part correlations revealed the DERS $(r=.28)$, rather than the ERICA $(r=-.06)$, was explaining the variance in NSSI severity. In spite of the strong correlation between the DERS and ERICA scores, inspection of the VIF value $(V I F=2.10)$ provided no evidence of multicollinearity. This suggests that, in this sample at least, the ERICA and the DERS are accounting for the same portion of variance in NSSI severity.

In summary, NSSI status can be distinguished in a similar manner using both the DERS and the ERICA. Both self-report measures show similar associations with NSSI severity, although regression analysis revealed that only the DERS was uniquely predictive.

\section{Practical Considerations}

The ERICA out-performs the DERS when taking into account practical considerations of conducting longitudinal research with adolescents. Vasilev and colleagues (2009) note that 
their sample of adolescents aged 11 to 15 years old were able to complete the DERS without assistance from researchers. In contrast, within the current sample, during data collection several younger participants (aged approximately 13 to 14 years old) asked for clarification of question meaning. Most common was confusion as to what 'wallowing" meant ("When I'm upset, I believe that wallowing in it is all I can do"). The inconsistencies compared to Vasilev and colleagues (2009) may be in part due to the larger sample size providing greater variability in reading levels ( $N=165$, compared to 647 in the current study). Vasilev and colleagues (2009) may also have had a greater level of selection bias in that the sample was recruited from a high socio-economic status area and participation required substantial buy-in from parents, such that the adolescents who participated may have higher reading ability than average. Future research is needed to disentangle these inconsistencies.

An additional practical consideration is that at 16 items ( 15 items used for analyses) the ERICA is substantially shorter than the 36-item DERS. When designing large survey batteries such as the Youth Wellbeing Longitudinal Study from which Study Two and Three are drawn, researchers need to consider the trade-off between the number of constructs being accurately measured and the appropriate length of time for participants to complete the survey. That is, unless the detail provided by the subscales is required, the ERICA out performs the DERS in terms of brevity.

\section{Limitations and Future Directions}

Although the sample was relatively large, it was drawn from a single, high-decile school, and so may not generalise to a larger population of New Zealand adolescents. It should also be noted that in comparison to recent New Zealand census data (Statistics New Zealand, 2013), our sample over-represents people identifying as New Zealand European/ Pākehā, (90.2\% in our sample vs $74.0 \%$ in the general population) and under-represents Māori (2.5\% in our sample vs $14.9 \%$ in the general population) and other minority ethnicities. Previous research has suggested that demographic variables such as culture may affect emotion regulation (Matsumoto, Yoo, \& Nakagawa, 2008; Raver, 2004). As such, future research should validate the ERICA and the DERS in adolescent samples with greater cultural and economic diversity.

\section{Summary and Conclusions}

The DERS and the ERICA were both administered to a large sample of community adolescents. Both emotion regulation scales demonstrated good internal reliability, and a 
factor structure similar to those previously reported. Although both the DERS and the ERICA showed subtle deviation from the original factor loadings reported, these differences were more apparent for the DERS. The DERS demonstrated a systematic gender difference, whereas the ERICA did not. Both scales replicated established associations with positive and negative aspects of wellbeing, as well as NSSI engagement and severity. Practical considerations of conducting a large scale survey favours the ERICA over the DERS. Therefore although the DERS is more widely used, Study One concludes that the ERICA is more appropriate for use in Study Two and Study Three.

\section{Study Two}

\section{Longitudinal test of the relationship between emotion regulation and NSSI}

Although limited in number, previous longitudinal studies supports aetiological models of NSSI such as the Experiential Avoidance Model (Chapman et al., 2006) and the Cognitive-Emotional Model (Hasking et al., 2016) by suggesting that disordered emotion regulation prospectively predicts subsequent NSSI (Fox et al., 2015). However, systems theory argues that dynamic interactions between an individual and their environment mould development (Sameroff \& Seifer, 1983). Researchers have speculated that engaging in NSSI may impair emotion regulation by creating additional intense negative emotions, reducing an individual's perceived mastery and ability to tolerate distress, and/or by damaging interpersonal relationships in which emotion regulation strategies are modelled and practised (Gratz, 2003). However, this reciprocal relationship has not yet been systematically examined. Using the emotion regulation measure validated in Study One (the ERICA), Study Two tracks the development of emotion regulation and NSSI in a cohort of young community adolescents over a period of three years. A cross-lag panel model is then used to test whether emotion regulation prospectively predicts NSSI, NSSI prospectively predicts emotion regulation, or whether emotion regulation and NSSI share a reciprocal, bidirectional relationship. Finally, given that Lundh and colleagues (2011) found that the bidirectional relationship between DSH and emotional wellbeing differed by gender, I shall test whether the longitudinal relationship between NSSI and emotion regulation differs systematically for girls and boys.

One of the fundamental challenges of longitudinal research is the choice of when to sample participants in order to track the change of interest (Ployhart \& Vandenberg, 2010). 
For many people who self-injure, NSSI is an adolescent-limited behaviour (Klonsky, 2011). The onset of NSSI typically ranges from 12 to 15 years old (Andover et al., 2010; Nixon, Cloutier, \& Jansson, 2008), prevalence peaks at approximately 15 to 16 and then begins to decline during young adulthood (Plener et al., 2015). Therefore, in order to capture the behaviour as it unfolds, rather than rely on retrospective report, it is necessary to draw from a sample of young adolescents.

Another important aspect of longitudinal research design is the choice of lag-time between waves of measurement so that there is sufficient time for change to occur, but not too much time that the change will have dissipated (Ployhart \& Vandenberg, 2010; Selig \& Little, 2012). Although previous longitudinal NSSI research with adolescents has used lags ranging from three months to 30 months (Guerry \& Prinstein, 2009; Hankin \& Abela, 2011a), research with community adolescents recruited from high schools has typically used a 12month lag (see for example, Lundh, Bjärehed, \& Wångby-Lundh, 2013; Prinstein et al., 2010; Tatnell et al., 2014). None of the longitudinal research with community adolescents explicitly states the rationale behind the choice of a 12-month lag, although Marshall, Tilton-Weaver and Stattin (2013) suggest that annual assessments are less invasive for participants and schools. I also speculate that time lags of fewer than 12 months would increase the difficulty of getting research buy-in from schools, parents, and participants. Therefore, a 12-month lag between waves is consistent with previous research and perhaps best meets practical considerations of longitudinal research in school settings.

A final design consideration is the number of waves to include within the longitudinal study. Three waves of measurement are often considered the minimum number for a longitudinal design (Chan, 1998; Ployhart \& Vandenberg, 2010). However, the majority of NSSI longitudinal research with community adolescents use only a two wave design (Lundh et al., 2013; Lundh et al., 2011; Martin, Thomas, Andrews, Hasking, \& Scott, 2014; Prinstein et al., 2010; Tatnell et al., 2014, although see Marshall et al., 2013 as an exception). Relying on only two waves of measurement prevents assessment of nonlinear changes, assessment of longitudinal mediational relationships, differences in rate of change, and potentially confounds true change and measurement error (Singer \& Willett, 2003; Polyhart \& Vandenberg, 2010).

In sum, taking into account both the theoretical and practical considerations of research suggests that an adequate method to test the relationship between the development 
of emotion regulation and NSSI would be a three wave longitudinal design with 12 month lags, with a sample of young adolescents.

\section{Method}

\section{Participants}

Participants were drawn from the Youth Wellbeing Study longitudinal survey, a threewave study following a cohort of New Zealand community adolescents examining the risk and preventative factors for NSSI. Students were surveyed at three time points, each approximately one year apart. In total, 972 students from 15 different secondary schools in the greater Wellington region completed the survey at Time One. Each participant was assigned a unique code which was used to match participants across the Time One (T1), Time Two (T2) and Time Three (T3) surveys. 477 participants were matched across all three time points, forming the final sample in the present study with $168(35.4 \%)$ participants identifying as male, $305(64.2 \%)$ as female, and two (>1\%) as transgender ${ }^{3}$. At T1, participants ranged from 12 to 15 years old $(M=13.56, S D=0.98)$. The majority of participants (74.3\%) primarily identified as Pākehā/New Zealand European, 5.5\% as Māori (indigenous New Zealander), 3.3\% as Pasifika (Samoan, Cook Island Māori, Tongan, or Niuean), $2.5 \%$ as Indian, $1.1 \%$ as Chinese, $8.4 \%$ as a non-listed ethnicity (e.g. South African), and $4.4 \%$ did not choose a primary ethnicity.

\section{Materials}

\section{Non-suicidal self-injury}

At each time point, all participants completed a screening question indexing NSSI lifetime history (see Appendix C). Participants who indicated that they had never thought about or engaged in NSSI at T1, T2, or T3 were assigned a Recent NSSI score of zero for that time point. At T1, participants who indicated they had engaged in NSSI were asked "When was the last time you did something to hurt yourself on purpose?" responding either 'Within the last week', 'Within the last month', 'Within the last year', and 'More than a year ago'. Participants who indicated that they had last engaged in NSSI more than a year ago were assigned a T1 Recent NSSI score of zero, whereas those who indicated their last NSSI episode was within a year were assigned a score of one. At T2 and T3, participants who indicated they had engaged in NSSI were asked "How many times have you hurt yourself

\footnotetext{
${ }^{3}$ As the number of adolescents who identified as transgender in the sample is too small to draw informative statistical conclusions, subsequent gender analysis will compare only participants who identified as either male or female.
} 
since the last survey", responding either 'Never', 'Once', 'A few times' or 'Many times'. Participants who indicated that within the last year, they had not engaged in NSSI episode were assigned a Recent NSSI score of zero, whereas those who indicated engaging in NSSI once or more within a year were assigned a score of one. Across all three time points, participants who reported thoughts of NSSI, but had not engaged in NSSI, were assigned a Recent NSSI score of zero.

At each wave, participants who reported a lifetime history of NSSI completed the Inventory of Statement About Self-Injury (ISAS; Klonsky \& Glenn, 2009) to assess the functions their self-injury serves. The ISAS is described in Study One (pp. 30-31) and is presented in full in Appendix E. The ISAS has previously demonstrated adequate internal consistency (Klonsky \& Glenn, 2009; Study One) and has been validated for use in adolescent samples (Kortge et al., 2013). Within the current sample, the ISAS showed adequate internal consistency at T1 $(\alpha=.82$ for both Intrapersonal Functions and Interpersonal Functions), T2 (Intrapersonal Functions $\alpha=.80$, Interpersonal Functions $\alpha=$ .75 ), and T3 ( $\alpha=.76$ for both Intrapersonal Functions and Interpersonal Functions), as well as adequate test-retest reliability from T1 to T2 (Intrapersonal Functions $r=.49$, Interpersonal Functions $r=.67$ ), and T2 to T3 (Intrapersonal Functions $r=.62$, Interpersonal Functions $r=.51$ ), indicating good reliability over time. The ISAS was administered primarily to assess the representativeness of the NSSI sample compared to international samples.

\section{Emotion regulation}

Participants completed the 16-item ERICA (MacDermott et al., 2010) described and validated in Study One (pp. 27, 39-49). As in Study One, the item "When others are upset, I become sad or concerned for them" did not correlate well with the other items, $r(475)=-$ .001 , and so was removed. Items scores were then totalled to provide an overall emotion regulation score. Similar to Study One, the 15-item ERICA showed good internal reliability at T1 $(\alpha=.82, M=3.81, S D=0.50), \mathrm{T} 2(\alpha=.82, M=3.74, S D=0.51)$ and T3 $(\alpha=.83, M=$ $3.73, S D=0.51$ ). The test-retest reliability of the ERICA was .63 from T1 to T2, and .74 from $\mathrm{T} 2$ to $\mathrm{T} 3$, indicating good reliability over time.

\section{Procedure}

Ethical approval for this study was granted by the National Health and Disability Ethics Committee (MEC/11/12/108). At the outset of the project, 45 secondary schools in the 
greater Wellington region were contacted and invited to take part in the study during 2011. Fifteen schools agreed to take part in the study. At Time 1 (T1) students in Year 9 and, at one school, Year 10 took home an information pack and consent form for parents. 1451 students returned a completed consent form, with $75.5 \%$ of students who returned a form having parental consent to take part in the study. Although a slightly lower overall rate of parental consent than reported in Study One, this rate is consistent with other longitudinal NSSI research recruiting from secondary schools which also required active parental consent (Andrews et al., 2014). In order to take part in the study, both parental consent and participant assent were required.

The survey was administered in 2012 and 2013 in classrooms under the supervision of a research team member(s) during a class period. Participants were first given an information sheet outlining the study and the opportunity to ask researchers any questions. Participants were informed that their participation in the current study was voluntary and they were able to withdraw at any time without consequence. Participants were also given assurance that their answers would remain confidential, but that if the team were concerned out their wellbeing then their name (but not answers) may be passed on to school pastoral care staff. The survey consisted of a series of questionnaire measures assessing youth wellbeing and took approximately 40 minutes to complete. Following participation, students were debriefed and given a list of contact details for community services, should they wish to seek support. Students were also able to approach team members following participation if they had any questions or concerns. Students also had the option of leaving their email or postal address to receive a summary of study results. The Time 2 (T2) and Time 3 (T3) surveys were conducted in a similar procedure approximately one year (Median days $=361$ ), and two years (Median days $=327)$ after the T1, respectively. Each participant was assigned a unique code, which was used to match participants across the T1, T2 and T3 surveys. Of the original T1 sample $24.7 \%$ were unable to be matched between Time 1 and Time 2, and 23.4\% were unable to be matched from $\mathrm{T} 2$ to $\mathrm{T} 3$, resulting in an overall retention rate of $52.1 \%$. This retention rate is comparable to other longitudinal research in New Zealand over three waves (60\%) with adult samples (Satherley et al., 2015).

As in Study One, missing data was imputed where possible in order to maximise the sample size available for subsequent analysis. Given that the ERICA shows adequate internal consistency, when participants with missing data had completed approximately $80 \%$ of the scale, the average of the items they had completed was calculated and inserted into the 
missing cells before a sum score was calculated. As suggested by Kenny (1975), any participants who were missing scale scores for one or more variables were deleted prior to analysis.

\section{Results}

\section{Descriptive Statistics}

Non-suicidal self-injury

Across all three waves the most common forms of NSSI were cutting, scratching, and punching or banging oneself. Within the same subsample, affect regulation and selfpunishment were consistently the most endorsed functions of life-time NSSI (see Appendix I). At T1 $16.2 \%$ of participants reported engaging in recent NSSI (i.e., within the past 12 months), $18.1 \%$ at T2 and $17.5 \%$ at T3. Prevalence of NSSI was higher among girls than boys across all time points; $20 \%$ vs. $9.4 \%$ at $\mathrm{T} 1\left(\chi^{2}(1)=9.01, p=.003\right), 23.9 \%$ vs. $7.6 \%$ at T2 $\left(\chi^{2}(1)=19.53, p<.001\right)$, and $23.3 \%$ vs. $7.1 \%$ at T3 $\left(\chi_{(1)}^{2}=19.92, p<.001\right)$.

Consistent with previous research demonstrating that NSSI is a highly unstable behaviour (Whitlock et al., 2011), the three waves captured a large degree of variance in NSSI trajectory. The majority of participants (71.6\%) reported no NSSI engagement. In contrast, $6.7 \%$ of participants reported maintenance of NSSI across all three time points. Capturing the onset of the behaviour, 5.9\% of participants reported engaging in NSSI at both $\mathrm{T} 2$ and T3 (T2 onset) and 4\% at T3 only (T3 onset). Capturing cessation of the behaviour, $5.5 \%$ of participants reported engaging in NSSI only at T1 (T2 cessation) and 3.2\% reported engaging at T1 and T2 (T3 cessation). Additionally, 2.3\% of participants reported engaging in NSSI at T2 only and $0.8 \%$ reported engaging in NSSI at T1 and T3.

\section{Emotion regulation}

In order to assess gender differences and monotonic changes in emotion regulation over time, a 3 X 2 mixed Analysis of Variance (ANOVA) was conducted with time as the within-subjects factor (Time: T1, T2, T3) and gender as the between-subjects factor (Gender: male, female). Mauchly's Test of Sphericity indicated that the assumption of sphericity had been violated, $\chi^{2}(2)=24.98, p<.001$. With Greenhouse-Geisser correction, a main effect of time was found, $F(1.90,899.63)=7.05, p=.001, \eta p^{2}=.02$. Follow-up Tukey post hoc tests revealed there was no difference in ERICA scores from T2 to T3, $t(474)=0.32, p=.748, d=$ .01. Contrary to expectations, participants at $\mathrm{T} 1(M=3.80, S D=0.50)$ reported higher ERICA scores than participants at T2 $(M=3.73, S D=0.51), t(474)=3.36 p=.001, d=.13$, 
and T3 $(M=3.73, S D=0.51), t(474)=3.51, p<.001, d=.14$, indicating a decrease in emotion regulation capacity over time. However, the effect sizes for these comparisons are small (Cohen, 1960), suggesting that these differences may not be particularly meaningful. Consistent with Study One, no evidence was found for a main effect of gender $(F(1,473)=$ $\left..14, p=.710, \eta p^{2}<.001\right)$, suggesting that males and females report similar levels of emotion regulation when assessed using the ERICA. No evidence for an interaction between gender and time was found, $F(1.90,899.63)=.56, p=.563, \eta p^{2}=.001$.

Zero-order correlations between NSSI, Emotion Regulation, and gender across all three time points are presented in Table 9. As predicted, NSSI and Emotion Regulation were negatively associated across all three time points. NSSI was positively associated with identifying as female, while gender was unrelated to Emotion Regulation.

Table 9

Zero-order correlations ( $r s$ ) between gender, NSSI and Emotion Regulation across the three time points in Study Two.

\begin{tabular}{lcccccc}
\hline Measures & 1. & 2. & 3. & 4. & 5. & 6. \\
\hline 1. Gender & & & & & & \\
2. T1-NSSI & $.14^{*}$ & & & & & \\
3. T1-Emotion Regulation & .02 & $-30^{*}$ & & & & \\
4. T2-NSSI & $.20^{*}$ & $.49^{*}$ & $-.27^{*}$ & & & \\
5. T2-Emotion Regulation & -.03 & $-.26^{*}$ & $.63^{*}$ & $-.33^{*}$ & & \\
6. T3-NSSI & $.21^{*}$ & $.34^{*}$ & $-.17^{*}$ & $.65^{*}$ & $-.30^{*}$ & \\
7. T3-Emotion Regulation & -.04 & $-.19^{*}$ & $.61^{*}$ & -.33 & $.74^{*}$ & $-.36^{*}$ \\
\hline
\end{tabular}

Note. Gender coded as $0=$ Male, $1=$ Female. ${ }^{*} p<.001 . N=475$. 
Figure 7. Proposed cross-lagged model in Study Two. Note. ………...... = cross-sectional /synchronous relationships, $-\cdot-\cdot-\cdot \cdot=$ stability relationships, and $=$ cross-lagged relationships.

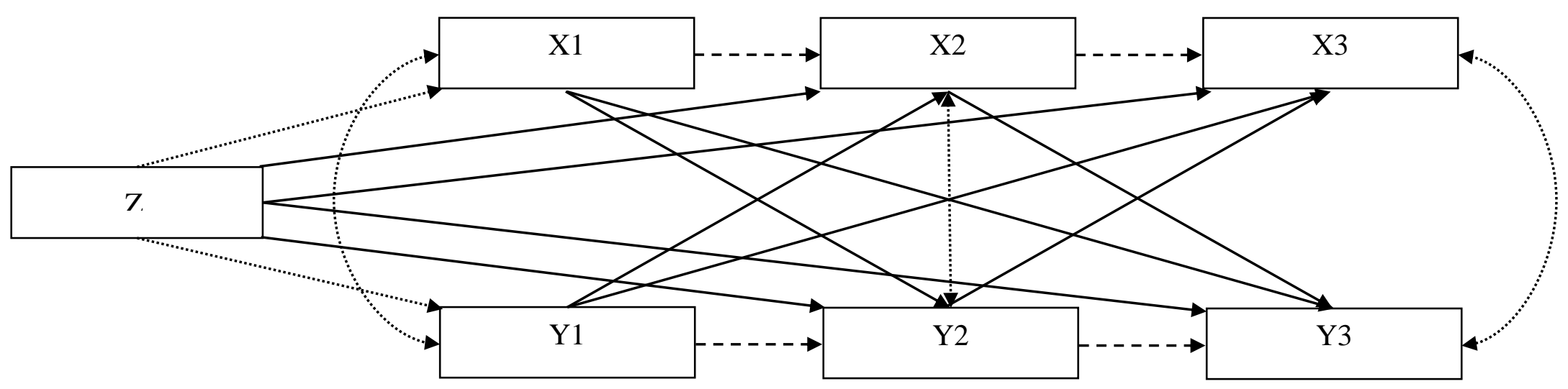

Figure 8. Standardised regression weights for the cross-lag model in Study Two. Note. $=$ significant pathway, significant pathway $(p>.05),{ }^{*} \mathrm{p}<.001,{ }^{\wedge} p$ s range from .036 to .002 . Gender coded as $1=$ male, $2=$ female.

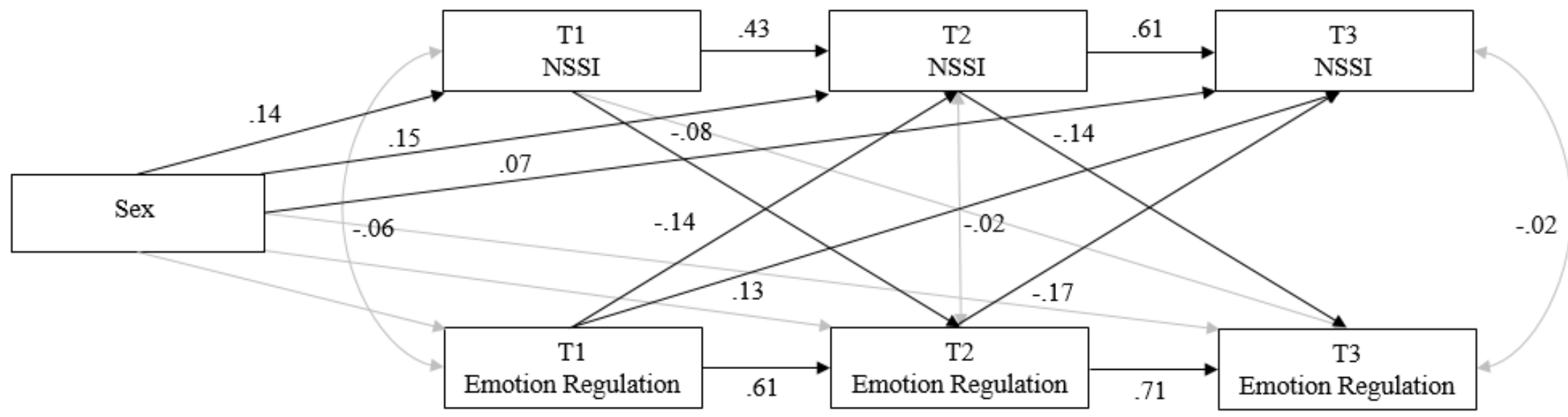




\section{Longitudinal Cross-lag Model}

AMOS version 23 (Arbuckle, 2014) was used to test the hypothesised bidirectional relationship between NSSI and emotion regulation using a cross-lag panel model. Cross-lag panel analyses predict change in constructs over time. Constructs (NSSI and Emotion Regulation) are measured at different time points (T1, T2, and T3). Later measurements are regressed onto earlier time points in order to predict change in the construct over time while accounting for prior levels of the construct being measured (Selig \& Little, 2012). That is, the cross-lagged coefficient between T1 Emotion Regulation and T2 NSSI controls for the relationship between T1 NSSI and T2 NSSI. Figure 7 presents the proposed cross-lagged model. This model tested the relationship between two constructs measured at three timepoints, generating six variables (X1, X2, X3 Y1, Y2, and Y3) and 13 coefficients. Three of these coefficients are cross-sectional; X1 and Y1, X2 and Y2, X3 and Y3. Ten of these coefficients are across time, four assessing the stability of the two constructs (X1 and X2, X2 and $\mathrm{X} 3, \mathrm{Y} 1$ and $\mathrm{Y} 2, \mathrm{Y} 2$ and $\mathrm{Y} 3$ ) and six which assess the cross-lagged relationships (X1 and $\mathrm{Y} 2, \mathrm{X} 1$ and $\mathrm{Y} 3, \mathrm{Y} 1$ and X2, Y1 and X3, X2 and Y3, Y2 and X3). Given that Lundh and colleagues (2011) suggested the relationship between NSSI and psychological factors may differ for boys and girls, gender was added to the model resulting in an additional two crosssectional coefficients ( $\mathrm{Z}$ and $\mathrm{X} 1, \mathrm{Z}$ and $\mathrm{Y} 1$ ) and four cross-lagged coefficients ( $\mathrm{Z}$ and $\mathrm{X} 2, \mathrm{Z}$ and $\mathrm{X} 3, \mathrm{Z}$ and $\mathrm{Y} 2, \mathrm{Z}$ and $\mathrm{Y} 3$ ). Note that although gender was measured at T1, neither NSSI nor emotion regulation can logically cause gender and so these cross-sectional relationships were modelled with single-headed arrows. By including gender in the model, the variance associated with gender is removed from the remaining relationships in the model. That is, gender as a potential third variable is included within the model.

Figure 8 shows the longitudinal cross-lagged model for NSSI and emotion regulation, with the addition of gender. Both NSSI and emotion regulation show large stability coefficients across both time lags. In terms of gender, identifying as female positively predicted T1 NSSI $(\beta=.14, p=.002)$, T2 NSSI $(\beta=.15, p<.001)$, and T3 NSSI $(\beta=.07, p=$ .036), but was unrelated to emotion regulation at any time point ( $p$ s range from .89 to .40 ). Consistent with our hypothesis, T1 NSSI negatively predicted T2 emotion regulation $(\beta=-$ $.08, p=.027)$ and T1 emotion regulation negatively predicted T2 NSSI $(\beta=-.14, p<.001)$. This bidirectional relationship was repeated over the subsequent one-year time lag, such that T2 NSSI negatively predicted T3 emotion regulation $(\beta=-.14, p<.001)$ and T2 emotion regulation negatively predicted T3 NSSI $(\beta=-.17, p<.001)$. T1 NSSI did not predict T3 
emotion regulation. Contrary to expectations, T1 emotion regulation positively predicted $\mathrm{T} 3$ NSSI $(\beta=.13, p=.003)$.

\section{Discussion}

Study Two investigated the direction of the relationship between NSSI and emotion regulation in a cohort of community adolescents. As expected within a community sample, the majority of participants did not engage in NSSI over the duration of the study, while others experienced onset, maintenance, cessation and resurgence of NSSI behaviour. A crosslagged model (with participant gender included) was constructed to predict changes in NSSI and emotion regulation across the three waves with two year lags. Consistent with predictions of a bidirectional relationship, emotion regulation negatively predicted NSSI a year later and NSSI negatively predicted emotion regulation a year later. Although identifying as female predicted engaging in NSSI, this bidirectional relationship was unrelated to gender.

This study further establishes emotion regulation as a risk factor for subsequent NSSI. Although previous longitudinal studies have investigated the development of emotion regulation and NSSI during adolescence, these have focused on specific aspects of emotion regulation, such as reappraisal processes (Andrews et al., 2014; Selby, Franklin, CarsonWong, \& Rizvi, 2013; Tatnell et al., 2014), negative emotionality (Hankin \& Abela, 2011a), and symptoms of emotional problems (Lundh et al., 2011). In contrast, Study Two used a measure of holistic emotion regulation to predict future NSSI, consistent with the hypothesis that poorer emotion regulation may underlie NSSI. Contrary to expectations, T1 emotion regulation was found to positively predict T3 NSSI, suggesting those who report greater emotion regulation at T1 are more likely to engage in NSSI at T3. Given that this positive relationship is not predicted by theory and that $\mathrm{T} 1$ emotion regulation is negatively correlated with T3 NSSI $(r=-.17)$, this relationship may be a statistical artifact resulting from the fact that a large proportion of the variance in T3 NSSI is already explained by T2 NSSI and T2 emotion regulation. Future research should establish that this positive relationship between emotion regulation and NSSI two years later is not attributable to spuriousness before any theoretical explanations are offered.

Study Two also clarifies our current understanding of NSSI development in adolescence by being the first to empirically demonstrate that the relationship between emotion regulation and NSSI is in fact bidirectional. Engaging in NSSI at T1 and T2 predicted subsequent decreases in emotion regulation a year later, suggesting that NSSI may cause damage to an individuals' emotion regulation skills. 
This bidirectional relationship is consistent with theoretical accounts of why people self-injure. The Experiential Avoidance Model (Chapman et al., 2006) argues that, in the context of sub-optimal emotion regulation abilities, an individual engages in NSSI in order to escape from overwhelming emotions. Relief from these adverse emotions (see for example, Franklin et al., 2010; Muehlenkamp et al., 2009) negatively reinforces the behaviour. In conjunction with habituation to the factors which constrain NSSI (e.g., fear of pain), and the paradoxical effects of experiential avoidance, this reinforcement creates a self-perpetuating cycle of NSSI. The Cognitive-Emotional Model (Hasking et al., 2016) also suggests that emotional reactivity, representations of NSSI, NSSI-related cognitions and representations of self are core risk factors which share a bidirectional relationship that develops over time.

Although the bidirectional relationship between emotion regulation and NSSI was previously untested, Lundh and colleagues (2011) found evidence for a bidirectional relationship between self-injury and psychological problems, including emotional problems, only in girls. Given that previous research has established gender differences in NSSI characteristics such as prevalence, forms and functions (Whitlock et al., 2011) as well as emotion regulation socialisation (Chaplin, Cole, \& Zahn-Waxler, 2005; Thompson \& Meyer, 2007), including gender in the Study Two model provides a more conservative test of the relationship between emotion regulation and NSSI. Identifying as female predicted subsequent NSSI, but in contrast to previous research the bidirectional relationship between NSSI and emotion regulation was evident for both girls and boys. Critically, this pattern of results suggests that the mechanisms underlying this bidirectional relationship operate in a similar manner for both girls and boys.

Similar to other longitudinal adolescent studies conducted over two years (Gandhi et al., 2017; Garisch \& Wilson, 2015), Study Two found NSSI engagement to be relatively unstable over three years. This is consistent with previous work indicating that some adolescents engage in NSSI only occasionally, whereas others may engage in NSSI repeatedly (Lundh et al., 2013; Whitlock et al., 2011). Inspection of the NSSI stability coefficients suggests that NSSI is more unstable from age 13 to $14(\beta=.43)$ than from age 14 to $15(\beta=.60)$, suggesting that prevention strategies may be most effective when targeted at 13 to14 year olds. 


\section{Clinical Implications}

Given that this research indicates that NSSI may damage emotion regulation and further reinforce vulnerability for NSSI, preventative interventions may be most clinically effective. Preventative strategies could be in the form of general emotion psychoeducation workshops for young people which focus on developing skills such as emotional awareness and clarity, willingness to experience emotions, or emotional acceptance (for example, Youth Wellbeing Study, 2017). Development of these skills may then act as scaffolding for the development of effective and contextually appropriate emotion regulation strategies. Similar psychoeducation for parents, teachers and youth workers may also be helpful in supporting them to effectively model these behaviours to adolescents. For current clients with NSSI, treatment should include a component targeted at improving and broadening the individual's emotion regulation skill set, and building an alternative set of adaptive behaviours to manage emotions without self-injuring (see for instance ERGT; Gratz \& Gunderson, 2006).

Assessment should include monitoring the client's emotion regulation strategies over time. Demonstrating atrophy of a client's skill-set may help to build motivation towards desistance. Incorporating the reciprocal relationship between emotion regulation and NSSI into psychoeducation of why some young people find NSSI hard to desist may help to validate the lived experience of the client, as well as to help parents to understand why their once resilient child has deteriorated since beginning NSSI. If a client presents with NSSI in addition to higher priority psychological problems, or is strongly resistant to desisting, mental health professionals should keep in mind that ongoing damage to sub-optimal emotion regulation skills may exacerbate other problems and so check in occasionally on NSSI. Additionally, clinicians should be on the lookout for factors that may bring about impaired emotion regulation in their client, such as social withdrawal or reduced belief in their ability to tolerate emotions. It may be necessary to with the client to mitigate the influence of these factors where possible.

\section{Limitations and Future Directions}

Although the cross-lagged longitudinal analysis provides evidence that the reciprocal relationship between emotion regulation and NSSI is not attributable to spuriousness, crosslagged analyses do not exclude the possibility that this bidirectional relationship may be explained by their mutual dependence on a third, unmeasured variable (Kenny, 2005) such as environmental stressors. Without direct experimental manipulation and random assignment it is difficult to establish irrefutable causality (Kenny, 1975; Selig \& Little, 2012). While future 
research could experimentally manipulate emotion regulation (e.g., via ERGT training Gratz \& Gunderson, 2006) in order to test some aspects of the model, in the case of NSSI ethical and practical limitations prevent true experimental manipulation.

An additional limitation of relying on cross-lagged analysis is that it describes the relationship modelled, rather than testing an explanation. In the case of emotion regulation predicting subsequent NSSI, current theoretical accounts offer explanations of how this change occurs (see for example, the Experiential Avoidance Model; Chapman et al., 2006; the Cognitive-Emotional Model: Hasking et al., 2016; and the Integrated Theoretcial Model: Nock, 2010). However at present it is not clear how NSSI leads to poorer emotion regulation. One such mechanism may be in the form of changes to representations of self, NSSI-related cognitions and representations of NSSI (see the Cognitive-Emotional Model; Hasking et al., 2016). For instance, previous work has demonstrated that engaging in emotional avoidance can create negative beliefs about the individual's perceived emotion regulation abilities (Salters-Pedneault et al., 2004). This poor self-efficacy belief may then affect an individual's willingness to attempt other emotion regulation strategies or to tolerate distress. Hence emotional avoidance serves to reinforce itself and impairs subsequent emotional functioning.

A second potential mechanism underlying the relationship between NSSI and poorer emotion regulation may be in the form and extent of interpersonal supports available to the young person. Many adolescents who self-injure report concerns about negative judgements and perceived stigma from their family and peers following disclosure (Fortune, Sinclair, \& Hawton, 2008), while others have reported experiencing humiliation and ostracism following disclosure (Harris, 2000). Fears of rejection and humiliation may prompt the young person to withdraw from social relationships that serve as supports. This increased social withdrawal limits the number of other emotion regulation strategies available to the young person, such as peer assisted distraction or problem solving, as well as creating fewer instances for a young person to learn about emotions and emotion regulation through social referencing and modelling (Morris et al., 2007). In addition to withdrawal from social relationships, the principle of homophily suggests that people associate or gravitate towards similar others and, once in a group, become more alike or emphasise their similarities (Brechwald \& Prinstein, 2011; Cohen, 1977). A young person who self-injures is more likely to be close friends with others who also engage in NSSI (see for example Claes, Houben, Vandereycken, Bijttebier, \& Muehlenkamp, 2010). That is, adolescents with NSSI may withdraw from parents and friends who they feel would not understand their behaviour, leaving them to rely on learning 
emotion regulation skills from peers whose own emotion regulation may also be poor. Study Three further explores how interpersonal relationships may underlie how the relationship between NSSI and subsequent emotion regulation.

\section{Conclusions}

Study Two suggests that approximately $17 \%$ of New Zealand adolescents have engaged in NSSI within the last year, emphasising the need to understand the development of the behaviour. A cross-lagged model with gender added was constructed to predict changes in NSSI and emotion regulation across the three years. Identifying as female was a risk factor for engaging in NSSI, but was unrelated to emotion regulation. Consistent with predictions of a bidirectional relationship, poor emotion regulation predicted subsequent NSSI and engaging in NSSI predicted subsequent impairments in emotion regulation.

\section{Study Three}

\section{Parental and peer attachment as potential mechanisms by which NSSI damages emotion regulation}

Study Two provides the first empirical evidence that engaging in NSSI may cause damage to a young person's emotion regulation skills. However, a key limitation of Study Two is that it simply describes the relationship and so cannot explain how this change occurs. In the discussion of Study Two, two possible mechanisms underlying the relationship between NSSI and subsequent poorer emotion regulation were proposed. In Study Three I test the hypothesis that changes in the quality of interpersonal relationships mediates the relationship between NSSI and subsequent emotion regulation.

Attachment is defined as the experience of psychological connectedness between individuals (Ainsworth, 1969), in this case the quality of the relationship between an adolescent and their parents, and an adolescent and their peers. Attachment is comprised of a number of different facets, such as interpersonal warmth, trust and the quality of communication (Armsden \& Greenberg, 1987). Attachment theory argues that attachment to parents forms the basis of other social relationships, such as close friendships and romantic relationships (Bowlby, 1982; for meta-analyses, see Benson, Mark, McWey, \& Ross, 2006; Gorrese \& Ruggieri, 2012). Strong attachment to parents and peers is a key aspect of adolescent wellbeing (see for example, Nada Raja, McGee, \& Stanton, 1992). 
The link between social relationships and the development of emotion regulation skills is well established. Family relationships play a particularly critical role in the development of emotion regulation skills during infancy and childhood (for reviews, see Calkins \& Hill, 2007; Thompson \& Meyer, 2007). Poor attachment during childhood leads to altered neuronal development and subsequent emotion dysregulation (Schore, 2001a, 2001b). During adolescence, emotion regulation skills continue to develop via social referencing and modelling by family and peers (Morris et al., 2007). In addition to fostering the development and mastery of emotion regulation skills, strong interpersonal relationships may facilitate effective emotion regulation processes such as reappraisal (e.g., a parent helping a young person see the silver lining in a unpleasant situation), distraction (e.g., playing video games with a friend), or labelling (e.g., discussing an emotional response with a friend). Impairment to interpersonal relationships may then damage the development and mastery of emotion regulation skills, as well as limit opportunities to engage in other emotion regulation strategies.

A functional analysis of NSSI suggests that people engage in NSSI in part to manage social relationships (Klonsky \& Glenn, 2009; Nock \& Prinstein, 2004). NSSI may function to facilitate relationships (e.g., communicate a need for care, or prevent a loved one from abandoning the young person) or to distance themselves from relationships (e.g. demonstrating that the adolescent is their own person, or create interpersonal boundaries in relationships). Together these interpersonal functions suggest that many people who selfinjure struggle to adaptively manage their social relationships.

Previous research using cross-sectional designs has established that NSSI is associated with poor interpersonal relationships in general (You, Leung, Fu, \& Lai, 2011), as well as poor family (Di Pierro, Sarno, Perego, Gallucci, \& Madeddu, 2012; Giletta, Scholte, Engels, Rutger, Ciairano, \& Prinstein, 2012; Martin et al., 2016; Muehlenkamp, Brausch, Quigley, \& Whitlock, 2013) and peer relationships in particular (Muehlenkamp et al., 2013). Preliminary longitudinal research suggests that poor relationship quality may be a risk factor for NSSI. Hankin and Abela (2011) report that adolescents' perceived lack of social support predicted the onset of NSSI over a two and a half year follow-up period. The quality of relationships with family seems to be particularly important. Low family adaptability and cohesion, as well as diminished family support, have been shown to predict subsequent engagement in NSSI from one to one and a half years later (Guerry \& Prinstein, 2009; Tatnell et al., 2014, although see Cox et al., 2012; Wilkinson, Kelvin, Roberts, Dubicka, \& Goodyer, 
2011). In fact, the quality of the relationships with family appears to be more important than the quality of relationships with peers. Both Andrews and colleagues (2014) and Tuisku and colleagues (2009) report that adolescents' perceived family support predicted subsequent engagement in self-injury, whereas perceived friend support did not.

Much less attention has focused on testing whether NSSI is a risk factor for impaired social relationships. Personal narratives suggest that, for some people, disclosure of selfinjury triggers intense negative reaction in others (Duggan, Heath, \& Hu, 2015; Gibb, Beautrais, \& Surgenor, 2010; Heath, Toste, \& Beettam, 2007; McAllister, Creedy, Moyle, \& Farrugia, 2002; McHale \& Felton, 2010). Fear that friends and family may react with negative judgement and stigma following disclosure is of concern to many young people who self-injure (Adler \& Adler, 2007; Fortune et al., 2008; Klineberg et al., 2013). In conjunction with the intense self-directed emotions of shame, disgust or guilt which follow self-injury (Laye-Gindhu \& Schonert-Reichl, 2005), this fear of social rejection may prompt an individual to withdraw from interpersonal relationships (see Gratz, 2003 for a similar argument). Disclosure of NSSI also creates intense negative emotions such as fear, guilt and anger within family and friends (Byrne et al., 2008). Family and friends may be ill-equipped to manage these emotions (McDonald, O’Brien, \& Jackson, 2007) and respond by distancing themselves from the young person or by invalidating the young person's experience, further damaging the quality of their relationship.

At present, empirical evidence that NSSI leads to subsequently poorer interpersonal relationships is limited and unreliable. Consistent with the idea that NSSI may damage social relationships, Tatnell and colleagues (2014) reported that adolescents who began self-injuring during the study period also reported decreases in support from family and friends. In a similar fashion, Burke and colleagues (2015) found that for adolescent girls (but not boys), NSSI prospectively predicted stressful interpersonal life events six months later. However, within a community sample of Chinese adolescents, relationship problems predicted NSSI a year later. Critically this relationship was not reciprocal - NSSI was unrelated to relationship problems a year later (You, Leung, \& Fu, 2012). In contrast, Hilt and colleagues (2008) found that although young adolescents with NSSI reported lower perceived relationship quality with both their mother and father than controls, at follow-up 11 months later, adolescents with NSSI reported an increase in the relationship quality with their fathers (but not mothers) whereas controls did not. Taken together, the evidence for how, and in which cases, NSSI affects interpersonal relationships is mixed and in need of further examination. 
Preliminary research has begun to look at the influence of relationship quality, and emotion regulation in conjunction when predicting NSSI. In a sample of adolescent girls admitted to a psychiatric hospital, Adrian and colleagues (2011) found that separately both family and peers relationship problems were directly related to NSSI as well as indirectly related through emotion dysregulation. In a longitudinal study of high school students over two years, Tatnell and colleagues (2014) found that the positive relationship between attachment anxiety and NSSI onset was partially mediated by cognitive reappraisal, suggesting that poor parental relationships are associated with emotion regulation impairments, which subsequently increases this risk of engaging in NSSI. However, to the best of my knowledge, Study Two is the first to empirically demonstrate that NSSI may damage subsequent emotion regulation skills. As such, previous research has not assessed the role of attachment in the relationship between NSSI and subsequent emotion regulation. In particular, it is not clear if the factors underlying the relationship from emotion regulation to NSSI also underlie the relationship from NSSI to emotion regulation.

More research is needed to better understand the complex interplay between interpersonal relationships, emotion regulation and NSSI. Study Three uses a quasilongitudinal multiple mediation model to test the hypothesis that engaging in NSSI is associated with poorer attachment to parents and peers, which in turn underlie subsequent emotion regulation ${ }^{4}$. Given the dearth of research exploring the relationship between NSSI and subsequent emotion regulation, Study Three tests whether Parental and Peer Attachment measured at T2 are possible mechanisms mediating the relationship between T1 life-time NSSI and T3 Emotion Regulation.

\section{Method}

Study Three data is also drawn from the Youth Wellbeing Study longitudinal survey described in Study Two. The sample descriptive, materials, study procedure, and longitudinal matching of participants is identical to that described in Study Two (pp. 52-55). Four hundred and thirty-seven participants $(68.3 \%$ identified as female, $31.1 \%$ as male, and $0.5 \%$ as transgender ${ }^{5}$ ) completed the measures used in Study Three analysis. NSSI severity was measured at T1, both parent attachment and peer attachment were measured at $\mathrm{T} 2$, and emotion regulation was measured at T3. That is, NSSI, attachment, and Emotion Regulation

\footnotetext{
${ }^{4}$ Note that, as Study Three uses the same dataset as Study Two, Study Three is not a replication of Study Two. ${ }^{5}$ Again, the number of adolescents who identified as transgender in the sample is too small to draw informative statistical conclusions. Subsequent gender analysis will compare only participants who identified as either male or female.
} 
are measured longitudinally, but Parent Attachment and Peer Attachment were measured at the same time point.

\section{Materials}

\section{Non-suicidal self-injury}

T1 NSSI life-time severity was measured in an identical manner to that described in Study One ${ }^{6}$. All participants completed a screening question assessing NSSI history (See Appendix C). Participants who indicated they had engaged in NSSI, or that they thought about doing so, then completed a modified version of simplified Deliberate Self-Harm Inventory (DSHI-s; Lundh et al., 2007) described in Study One (pp. 30; Appendix D). Consistent with previous research (Di Pierro et al., 2012), participants' responses to the 13 descriptions of NSSI behaviours (ranging from 0 - "I've never thought about doing this" to 4 - "I've done this many times") were averaged to provide an index of NSSI severity. Participants who indicated on the NSSI screening question that they had never thought about or engaged in NSSI were assigned a DSHI-s score of zero. The DSHI has previously showed good internal consistency ( $\alpha=.82$, Gratz, 2001) and the simplified version been validated for use in adolescent samples (Lundh et al., 2007; Study One). Within the current sample, the DSHI-s showed excellent internal consistency, $\alpha=0.84$ ).

Participants who reported a lifetime history of NSSI also completed the Inventory of Statement About Self-Injury (ISAS; Klonsky \& Glenn, 2009) described in Study One (pp. 30-31) and is presented in full in Appendix E. The ISAS indexes the functions a person's self-injury serves and was administered primarily to assess the representativeness of the NSSI sample compared to international samples.

\section{Attachment to Parents and Peers}

T2 attachment to parents and peers was measured using a shortened version of the Inventory of Parent and Peer Attachment (IPPA; Armsden \& Greenberg, 1987). Broadly, the IPPA measures adolescents' perceptions of psychological security in their relationships with parents and close friends across three dimensions; degree of mutual trust, quality of communication, and the extent of anger and alienation. Similar to previous research (Laible,

\footnotetext{
${ }^{6}$ Study Two operationalised NSSI as 'recent NSSI within the past 12 months', rather than 'NSSI lifetime severity' as it is in Study Three. Both measures of NSSI were completed throughout the three waves of the Youth Wellbeing Study. Given that the cross-lagged analysis in Study Two predicted change in NSSI over two lags, 'recent NSSI' was a better operationalisation of NSSI than 'lifetime history' (which theoretically could only ever increase). In contrast, Study Three assesses NSSI only at T1. In order to increase the variability of the NSSI measure for mediation analysis, 'NSSI lifetime severity' was preferred (as 'recent NSSI' is a binary measure).
} 
Carlo, \& Raffaelli, 2000), the current study included 12 items indexing parent attachment and 12 items indexing peer attachment. Participants respond to items such as "My parents accept me as I am" (parent attachment subscale item) and "I tell my friends about my problems and troubles" (peer attachment subscale item) on a 5-point Likert scale ranging from 1 - Never true to 5-Always true. For parental items, participants were instructed "if you have more than one person acting as either your mother or father (e.g., step-parents, or a member of your whānau who look after you like a parent) please answer the questions for the one you feel has most influenced you". See Appendix I for the full measure. The IPPA has been widely used to measure adolescent attachment within broader wellbeing research (see for example, Abela et al., 2005; Laible et al., 2000; Nada Raja et al., 1992), demonstrating good internal reliability (parent attachment $\alpha=.85$, peer attachment $\alpha=.84$; Laible et al., 2000), test-retest reliability and construct validity (Armsden \& Greenberg, 1987; Laible et al., 2000). In the current study, both parent and peer subscales showed excellent internal reliability $(\alpha=.84$ and .89 , respectively).

\section{Emotion Regulation}

T3 emotion regulation was measured using the ERICA (MacDermott et al., 2010) previously described in Study One and Study Two. The ERICA was validated for adolescent NSSI research in Study One (pp. 39-49), while the psychometric properties of ERICA within the current sample as well as the descriptive states for T3 ERICA are reported in Study Two (pp. 53).

\section{Results}

\section{Descriptive Statistics}

Non-suicidal self-injury

Among participants who reported a lifetime history of NSSI, cutting scratching and punching or banging oneself, were the most commonly endorsed behaviours, and affect regulation and self-punishment were the most commonly reported NSSI functions ${ }^{7}$. As expected, NSSI severity (Mean $=0.15, S D=0.39$, Medium $=0.00$ ) was non-normally distributed with skewness of $3.81(S E=.12)$ and kurtosis of $18.18(S E=0.23)$.

\footnotetext{
${ }^{7}$ In order to facilitate comparisons across samples, endorsement of rates of the different NSSI behaviours and functions are presented in Appendix J.
} 


\section{Attachment to Parents and Peers}

To assess for systematic differences in attachment to parents and peers as well as potential gender differences, a 2 X 2 mixed Analysis of Variance (ANOVA) was conducted with attachment target as the within-subjects factor (Attachment: Parental Attachment, Peer Attachment) and gender as the between-subjects factor (Gender: male, female). A main effect of attachment was found, $F(1,431)=13.85, p<.001, \eta p^{2}=.03$, whereby attachment to parents $(M=3.77, S D=0.84)$ was significantly higher than attachment to peers $(M=3.70$, $S D=0.74)$. No evidence was found for a main effect of gender, $F(1,431)=.55, p=.457, \eta p$ $2<.01$. However, these main effects were qualified by an interaction between attachment and gender, $F(1,431)=28.34, p<.001, \eta p^{2}=.06$. Consistent with previous research (Gorrese \& Ruggieri, 2012), post hoc Tukey tests showed that boys rated their attachment to peers $(M=$ $3.52, S D=0.73)$ lower than their attachment to parents $(M=3.88, S D=0.79), t(135)=5.76$ $p<.001, d=.49$. In contrast, girls reported no difference between their attachment to parents $(M=3.72, S D=0.86)$ and peers $(M=3.78, S D=0.74), t(296)=-1.40, p=.163, d=.08$.

Given that Study Two demonstrated that the relationship between NSSI and subsequent emotion regulation was similar for both boys and girls and no a priori gender specific differences were hypothesised, I decided against splitting subsequent analyses by gender.

Table 10 shows the zero-order correlations between T1 NSSI, T2 Parental Attachment, T2 Peer Attachment, and T3 Emotion Regulation. As predicted by attachment theory (Bowlby, 1982), T2 Parental Attachment and T2 Peer Attachment shared a strong positive association. As reported in Study Two, T1 NSSI was negatively associated with T3 emotion regulation. Replicating previous research (Di Pierro et al., 2012; Martin et al., 2016; Muehlenkamp et al., 2013), T1 NSSI was also negatively associated with T2 Parental Attachment and T2 Peer Attachment. Both measures of attachment were also strongly positively associated with T3 Emotion Regulation. Online computer software (Lee \& Preacher, 2013) was then used to test for differences between these dependent correlations. Controlling for the association between T2 Parental Attachment and T2 Peer Attachment, T2 Parental Attachment was associated in a similar manner to NSSI Severity $(Z=1.97, p=.049)$ and T3 Emotion Regulation $(Z=0.02, p=.490)$ as was T2 Peer Attachment. 
Table 10

Zero-order correlations between T1 NSSI Severity, T2 Parental Attachment, T2 Peer Attachment, and T3 Emotion Regulation in Study Three

1.

1. T1 NSSI Severity

2. T2 Parental Attachment

3. T2 Peer Attachment

4. T3 Emotion Regulation

Note. $N=437 .{ }^{*} p<.001$
2.

3.

\section{Longitudinal Multiple Mediation Analysis}

In order to assess whether the simultaneous effect of both T2 Parental Attachment and T2 Peer Attachment mediates the relationship between T1 NSSI Severity and T3 Emotion Regulation, a multiple mediation analysis was conducted using PROCESS (see Figure 9 for the proposed multiple mediation model). PROCESS is a conditional process modelling program which allows researchers to examine multiple moderators and mediators simultaneously (Hayes, 2012). An important feature of the model is that it simultaneously assesses the indirect effect of T1 NSSI $(X)$ on T2 Emotion Regulation $(Y)$ through T2 Parental Attachment $\left(M_{1} ; a 1, b 1\right)$; the indirect effect of T1 NSSI $(X)$ on T2 Emotion Regulation $(Y)$ through T2 Peer Attachment $\left(M_{2}: a 2, b 2\right)$; the indirect effect of T1 NSSI $(X)$ on T2 Emotion Regulation $(Y)$ through both T2 Parental Attachment $\left(M_{1}\right)$ and T2 Peer Attachment $\left(M_{2}\right)$ in serial $(a 1, d 21, b 2)$; and the direct effect of T1 NSSI $(X)$ on T2 Emotion Regulation $\left(Y: c^{\prime}\right)$. Bootstrapping with 5000 samples provided $95 \%$ confidence interval around the indirect effect in order to determine if the mediating effect is different from zero (Preacher \& Hayes, 2008).

The combined indirect effect of T2 Parental Attachment and T2 Peer Attachment on the relationship between T1 NSSI Severity and T3 Emotion Regulation was significant, $R^{2}=.28, F(3,433)=57.10, p<.001$, indicating that the combined set of mediators partially explained the relationship between T1 NSSI Severity and T3 Emotion Regulation. Both T2 
Figure 9. Proposed multiple mediation model in Study Three.

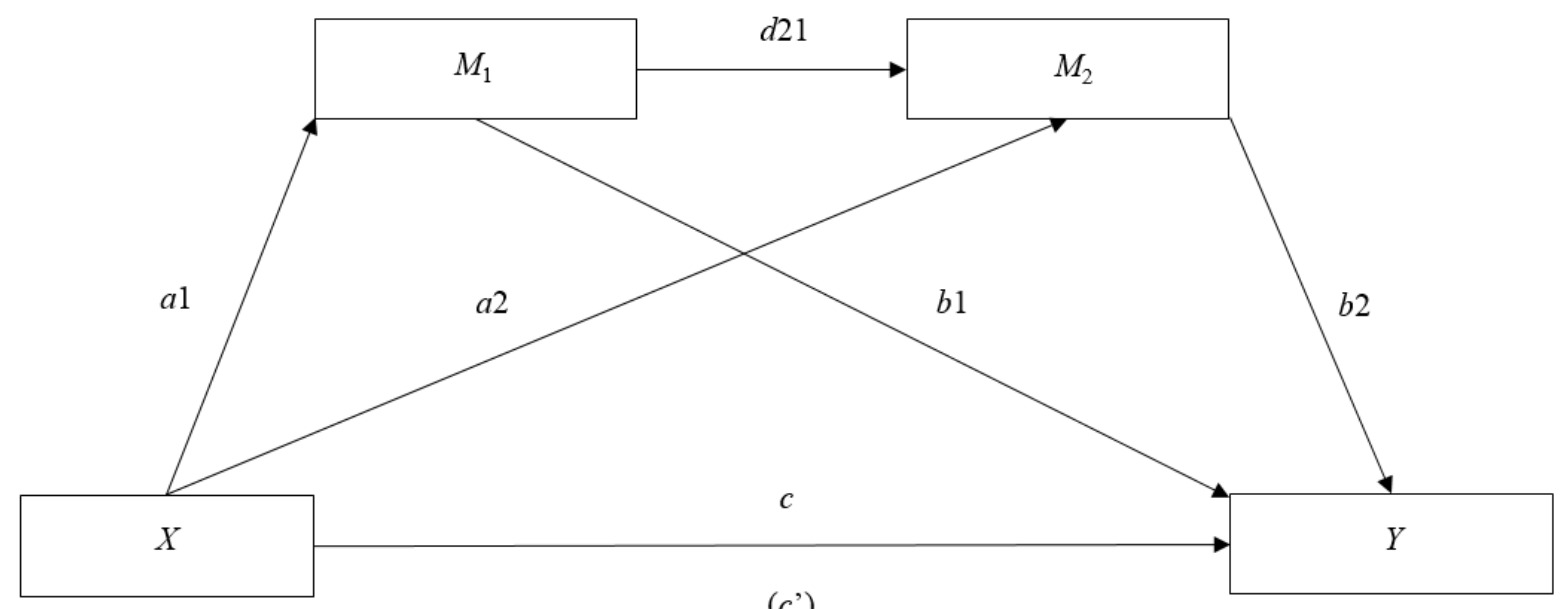

$\left(c^{\prime}\right)$

Parent attachment, $t(433)=5.81, p<.001,95 \%$ CI $[0.12,0.23]$, and T2 Peer Attachment $t(433)=6.22, p<.001,95 \%$ CI $[0.14,0.27]$, independently mediated the effect of T1 NSSI Severity on T3 Emotion Regulation. When taking into account both mediating variables, the direct pathway between T1 NSSI and T3 Emotion Regulation became non-significant, $t(434)$ $=-1.81, p=.071,95 \% \mathrm{CI}[-0.21,-0.01]$, suggesting that the relationship between T1 NSSI and T3 Emotion Regulation is fully mediated by both T2 Parental Attachment and T2 Peer Attachment. In addition, T2 Parental Attachment ( $b=-.70, p<.001,95 \%$ CI $[-0.89,-0.51])$ fully mediated the relationship between T1 NSSI and T2 Peer Attachment, $(b=-.15, p=.007$, $95 \%$ CI $[-0.31,0.01])$. See Figure 10 for the unstandardized parameter estimates for each of the direct and indirect pathways.

\section{Discussion}

In Study Three I tested the hypothesis that poorer attachment to friends and family is a mechanism through which engaging in NSSI 'damages' emotion regulation. Using threewave longitudinal data from a cohort of adolescents previously described in Study Two, Study Three indicates that T2 Parental Attachment and T2 Peer Attachment fully mediated the negative relationship between T1 NSSI Severity and T3 Emotion Regulation, suggesting that NSSI may cause damage to interpersonal relationships which underlie subsequent development of emotion regulation skills. 


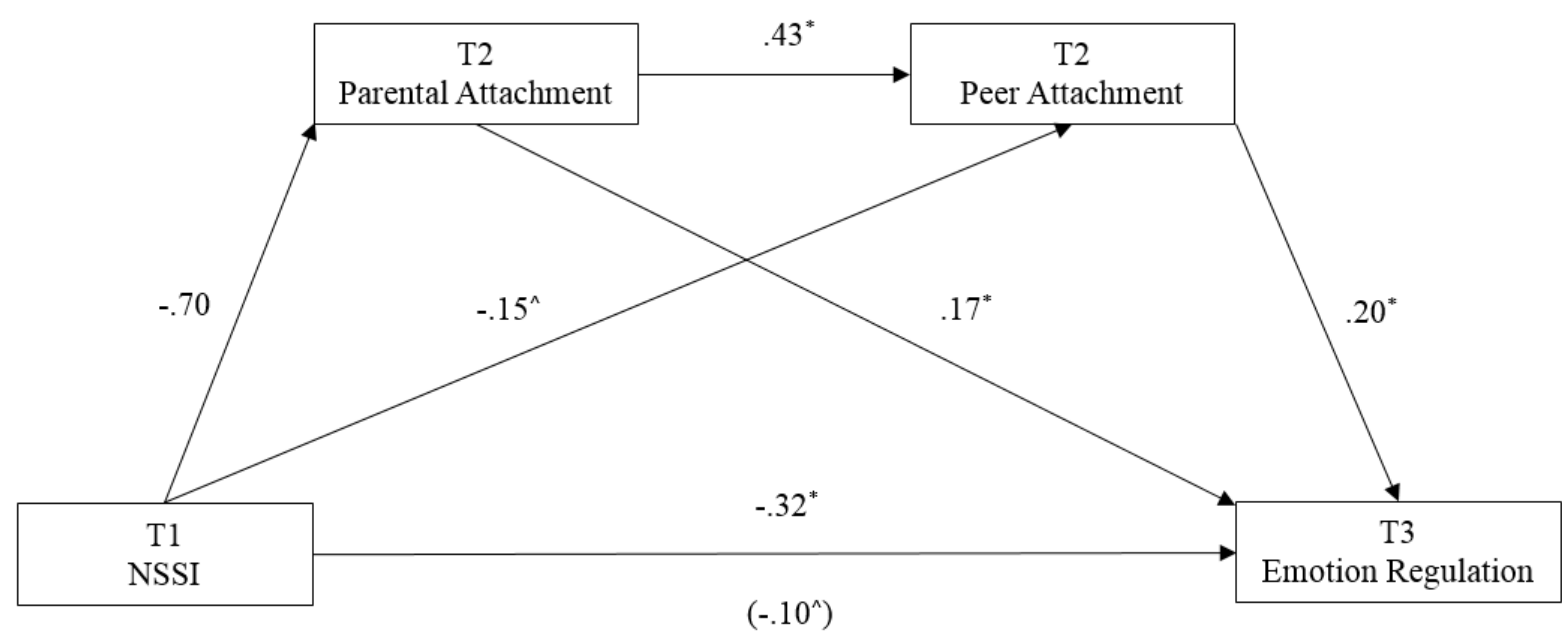

Figure 10. Parameter estimates for the direct and indirect effects within multiple mediation model in Study Three. Note ${ }^{*} p<.001, \hat{p}=.07, N=437$.

Study Three adds to the established literature emphasising the importance of parent and peer attachment for adolescent wellbeing. Consistent with attachment theory (Bowlby, 1982), attachment to parents and friends were strongly associated with each other $(b=.43, p$ $<.001,95 \% \mathrm{CI}[0.35,0.51])$. That is, experiences of trust, open communication, and acceptance (Armsden \& Greenberg, 1987) within parental relationships largely generalised to experiences of trust, open communication, and acceptance within friendships. Although Study Three measured attachment to parents and attachment to peers at the same time period, the strong association between the two is consistent with a wealth of previous research demonstrating that interactional style with close friends is strongly influenced by parental relationships (Eisenberg et al., 2001; Engels, Rutger et al., 2001). However, what is critical is that even when accounting for the relationship between parental and peer attachment, both factors were positively associated with subsequent emotion regulation. That is, attachment to parents $(b=.17, p<.001,95 \% \mathrm{CI}[0.125,0.23])$ and peers $(b=.20, p<.001,95 \% \mathrm{CI}[0.14$, $0.27]$ ) independently predicted better emotion regulation, suggesting that the emotional support of parents and friends, although overlapping, may play different roles. Perhaps parents have a greater influence on foundational aspects of emotion regulation, such as willingness to experience emotions, and scaffold cognitively demanding processes such as reappraisal or labelling of complex emotions (e.g. nostalgia). In comparison, peers may play a stronger role in normalising emotional experiences and modelling strategies to implement emotion regulation processes (e.g., labelling via listening to emotive music). Although 
relationships are an important support and facilitator of emotion regulation, the finding that attachment to parents and peers fully mediated the relationship between NSSI and subsequent emotion regulation is notable. Emotion regulation is not exclusively determined by interpersonal relationships with others (see for example, Hariri \& Holmes, 2006), so the fact that relationship attachment was found to fully explain the relationship between NSSI and subsequent emotion regulation speaks to the primacy of these parental and peer relationships during early adolescence.

Previous research assessing interpersonal relationships and NSSI has focused almost exclusively on the idea that poor interpersonal relationships are risk factors for NSSI. Study Three suggests that relationships may in fact be bidirectional, whereby engaging in NSSI may also damage interpersonal relationships. This finding is consistent with the personal narratives of people who self-injure, who often fear that NSSI discloure may damage their relationships with friends and family (Adler \& Adler, 2007; Fortune et al., 2008; Klineberg et al., 2013), and with empirical research where NSSI predicted interpersonal stressors six months later (Burke et al., 2015). However, this finding contrasts other large sample longitudinal research which reports no effect of NSSI on relationship problems six months later (You et al., 2012). However, these inconstencies may reflect cross-cultural differences in normative attachement styles (Van IJzendoorn \& Kroonenberg, 1988), where on average Chinese samples (such as You et al., 2012) reported poorer attachment than do USA samples (such as Burke et al., 2015). In addition, Burke and colleagues (2015) and You and colleagues (2012) both operationalized relationship quality by indexing both friends and family stressors. However, Study Three demonstrated that NSSI directly predicts poorer parental attachment, and indirectly predicts peer attachment via parental attachment. Perhaps NSSI has a stronger relationship with parents than friends because peers are more familiar with NSSI. In recent times, most adolescents report knowing somebody who self-injures (Claes et al., 2010). However, anecdotally this was not the case in previous decades. Therefore, NSSI may be more foreign and less understandable to parents than to peers. This lack of understanding may then contribute towards decreasing relationship quality to a greater extent for parents than for peers.

In contrast, longitudinal research by Hilt and colleagues (2008) found that engaging in NSSI predicted increased quality of relationships with fathers only, suggesting that it may be necessary to distinguish between maternal and paternal relationships. The functional model of NSSI also argues that the interpersonal functions of NSSI serve to modify interpersonal 
relationships (Klonsky, 2007; Nock, 2010) and is reinforced either positively (e.g., providing care) or negatively (e.g., preventing a loved one from abandoning the young person). Taken together, this pattern of results suggests that the interplay between interpersonal relationships and NSSI is likely to be complex and dynamic. Perhaps in the short term disclosure of NSSI triggers greater support and care from friends and family, but over time, persistence of NSSI results in frustration and guilt for not being able to stop the behaviour, and empathy burnout among family and friends (Byrne et al., 2008; McDonald et al., 2007). Future qualitative research with parents and friends of young people with NSSI, as well as longitudinal designs with multiple waves over short durations, may assist in better understanding the influence of NSSI on interpersonal relationships.

\section{Clinical Implications}

Clinically, Study Three suggests that if the negative influence of NSSI on parental and peer attachment can be mitigated or reduced, then the negative effect of NSSI on emotion regulation skills may be prevented. Assessment should include monitoring interpersonal relationships, particularly when addressing NSSI is not the highest treatment priority. When working with adolescents who self-injure, it may be beneficial to focus on the parental and peer relationships which are important to the adolescent and work to develop greater trust, communication and warmth within these relationships, for instance the interpersonal effectiveness module of DBT (Linehan, 1993). It may also be helpful to work with the young person and their caregivers to identify what factors are preventing these relationships from growing. However, it is important to note that poor relationship quality may be largely driven by (mis)perception, so it may be necessary to work with the client to improve how they view their relationships with key others. When working with parents and whānau, it is also important to acknowledge the difficulty of caring for a young person going through tough times, while also sitting with their own intense emotions. Psychoeducation focused on explaining NSSI as a functional emotion regulation behaviour may help parents and whānau to better understand why their child self-injures, and more effectively manage their own emotions, such as fear or sadness. An emphasis on the importance of parental self-care may also be effective.

\section{Limitations and Future Directions}

A key limitation of Study Three is that multiple mediation analysis does not control for change in a variable across time due to spuriousness. Given that previous research has established that poorer interpersonal relationships are a risk factor for NSSI (Cox et al., 2012; 
Tatnell et al., 2014, although see Wilkinson et al., 2011), it is also necessary to test if these two factors share a reciprocal relationship. Secondly, both measures of attachment were assessed at the same time point. Although T2 Parental Attachment fully mediates the relationship between T1 NSSI and T2 Peer Attachment, the causal inference of this relationship is based on the previous research (Benson, Mark et al., 2006; Gorrese \& Ruggieri, 2012) rather than longitudinal measurement in Study Three. Finally, the multiple mediation focuses only on the relationship whereby NSSI predicts poorer emotion regulation and ignores the complexity of the reciprocal relationship described in Study Two. However, this analysis would require a great deal of statistical power in order to avoid Type II error. Future research could address these limitations by using four waves of large-scale longitudinal data to conduct a cross-lagged panel analysis of NSSI, emotion regulation, parental attachment and peer attachment over time.

At present, the field's understanding of how, and in which instances, NSSI impacts interpersonal attachment is relatively rudimentary. Future multi-wave longitudinal research is needed to better elucidate the nature of this relationship. Consistent with previous research (Andrews et al., 2014; Tuisku et al., 2009), Study Three suggests that it may be fruitful to distinguish between parental and peer relationships, while other work suggests distinguishing between maternal and paternal relationships (Hilt et al., 2008). A key limitation of Study Three attachment to parents and peers was measured by asking only one half of the dyad to evaluate the relationship, so we are unable to distinguish between the 'actual' relationship quality and the adolescent's perceived relationship quality. Indeed, previous research suggests that adolescents and their parents differ in their perception of the same relationship (Baetens et al., 2014). Future research using adolescent-, parent-, and peer-reported measures would be well placed to disentangle the effects of 'actual' and perceived relationship quality. However, it is likely that perception rises out of reality and, in terms of NSSI risk, the distinction may not matter. Qualitative research with adolescents who self-injure may also be beneficial in teasing out whether NSSI impacts relationships equally - perhaps some relationships are strengthened while others are weakened and others do not change - as well as the process by which this change occurs.

Finally, although attachment fully mediated the relationship between NSSI and subsequent emotion regulation, it is unlikely that interpersonal relationships are the only mechanism. The finding from Study Two may also reflect a cognitive mechanism whereby, drawing from the Cognitive-Emotional Model and the Experiential Avoidance Model 
(Chapman et al., 2006; Hasking et al., 2016), engaging in NSSI changes to an individual's perception of themselves as being able to tolerate emotional distress and their perceived ability to use other emotion regulation strategies. Over time, NSSI becomes the habitual strategy with which to manage intense emotional distress. This reliance on NSSI then results in fewer opportunities to practise other emotion regulation skills and develop mastery and self-efficacy. Future research could test this hypothesis alongside attachment to friends and family to build a more complete understanding of how NSSI impairs subsequent emotion regulation.

\section{Conclusions}

Study Three used a quasi-longitudinal multiple mediation analysis to test whether attachment to parents and peers may mediate the relationship between NSSI and subsequent poorer emotion regulation. Preliminary evidence suggests that NSSI may directly damage parental attachment, while indirectly damaging peer attachment via parental attachment. Critically, damage to these key interpersonal relationships led to reduced emotion regulation a year later.

\section{General Discussion}

Theoretical frameworks of NSSI such as the Experiential Avoidance Model (Chapman et al., 2006), the Integrated Theoretical model (Nock, 2010) and the CognitiveEmotional Model (Hasking et al., 2016) argue that poor emotion regulation skills are a key factor underlying why some adolescents, without suicidal intent, deliberately engage in behaviours that cause direct injury to themselves. Longitudinal investigations offer the best opportunity to establish emotion regulation as a risk factor for NSSI engagement. Previous longitudinal assessments of the developmental relationship between emotion regulation and NSSI are preliminary, and limited to only two waves. In addition, despite speculation that engaging in NSSI may in turn impair emotion regulation (Gratz, 2003), this relationship has remains largely untested. In this thesis I tested the developmental relationship between emotion regulation (measured using the ERICA; MacDermott et al., 2010) and NSSI in a cohort of adolescents across three years. Study One established the validity of the ERICA for use in adolescent New Zealand samples by establishing the factor structure, the internal reliability, and convergent validity against the more widely used DERS. Study Two then used the ERICA to test the specific nature of the relationship between emotion regulation and NSSI using cross-lagged longitudinal analysis, finding evidence that these factors share a reciprocal relationship. Given that Study Two is the first to empirically demonstrate that 
engaging in NSSI predicts poorer emotion regulation, the mechanisms underlying this relationship were speculative. Study Three found support for the hypothesis that changes to the interpersonal support systems which support emotion regulation development mastery may be a mechanism by which NSSI 'damages' emotion regulation.

\section{Broad Contributions to NSSI Literature}

In addition to establishing the validity of the ERICA, Study One demonstrated that adolescents who reported thoughts of engaging in NSSI report similar levels of poor emotion regulation as do adolescents who engage in NSSI, suggesting that the two groups share similar levels of 'risk'. At present, people with thoughts, but not actions, of NSSI have received very little investigation, although as a group they may be at increased risk of NSSI engagement (Hasking, Andrews, \& Martin, 2013; Martin et al., 2011). Consistent with previous research (see for example, Fox et al., 2015; Whitlock et al., 2015), Study Two demonstrated that emotion regulation prospectively predicted NSSI behaviour, providing evidence that poor emotion regulation is a risk factor for the development of NSSI among adolescents. Study Two and Three demonstrate that engaging in self-injury predicts long term change, highlighting the importance of investigating the transition an individual takes from NSSI thoughts to NSSI behaviours. Future research is needed to establish how, and in what circumstances, emotion regulation increases the risk of NSSI.

Study Two and Study Three also add to the burgeoning literature assessing the effect of NSSI on other psychological factors. Given that NSSI as a field of study is only several decades old (Favazza \& Conterio, 1988; Nock, 2010), it is understandable that the vast majority of research within this field has focused on better understanding the psychological factors which predict NSSI (see for example, Lundh et al., 2011; Prinstein et al., 2010; Tatnell et al., 2014) rather than establishing the effect of NSSI on one's psychological wellbeing. Study Two and Study Three emphasise that by engaging in NSSI, an individual changes their environment in ways which may change their NSSI risk. Together with previous research demonstrating that engaging in NSSI affects identity development (Gandhi et al., 2017), pain thresholds (Koenig et al., 2017), parental relationships (Hilt, Nock, LloydRichardson, \& Prinstein, 2008), resilience, mindfulness and anxiety (Garisch \& Wilson, 2015), this thesis begins to explain how engaging in NSSI may affect subsequent development. 
Although not a specific focus of the research programme presented here, this thesis also provides more nuanced description of the instability of NSSI behaviour over time. It is well established that NSSI is a reasonably unstable behaviour; for instance, $25 \%$ of young adults with a life-time history of NSSI reported that they had engaged in NSSI only once (Whitlock, Eckenrode, \& Daniel, 2006). Previous literature has worked to establish life-time prevalence rates of NSSI (Muehlenkamp et al., 2012; Swannell et al., 2014), as well as 12month prevalence (Plener et al., 2015). However, rate of onset, cessation and maintenance of NSSI during adolescence is less well understood. Tatnell and colleagues (2014) reported that in the span of a year, $88.1 \%$ of their adolescent sample did not self-injure, $3.9 \%$ reported onset of NSSI, $4.2 \%$ cessation, and $4.2 \%$ reported maintenance of NSSI. Over a similar period, Gandhi and colleagues (2017) reported that $83.9 \%$ of their adolescent samples did not engage in NSSI, $2.8 \%$ reported onset of NSSI, $8.4 \%$ cessation, and $5.3 \%$ reported maintenance of the behaviour. However, both Tatnell and colleagues (2014) and Gandhi and colleagues (2017) have a large age range within their samples (12 to 18 years, and 12 to 19 years, respectively). Given the prevalence of recent NSSI varies by age (Plener et al., 2015), both studies potentially conflate age with NSSI stability. In comparison, the longitudinal data presented in Study Two and Study Three follows a cohort of adolescents aged 13-14 years old over two years; $71.6 \%$ did not self-injure, $5.9 \%$ reported onset of NSSI at T2, $4.0 \%$ onset at $\mathrm{T} 3,5.5 \%$ reported cessation at $\mathrm{T} 2,3.2 \%$ reported $\mathrm{T} 3$ cessation, and $6.7 \%$ reported maintenance of NSSI across three years. Additionally, three waves of longitudinal data better captures adolescents who experiment with NSSI (2.3\% of participants reported engaging in NSSI at T2 only) and those who relapse (0.8\% reported engaging in NSSI at T1 and T3, but not T2). Compared to the rates reported by Tatnell and colleagues (2014) and Gandhi and colleagues (2017), the longitudinal sample within this thesis reported greater onset of NSSI, but similar levels of cessation and maintenance. Drawing across studies, the higher onset rate within the younger sample leads me to speculate that processes which underlie the onset of NSSI may vary by age (e.g., peer influence, Prinstein et al., 2010) while the processes which underlie cessation and maintenance remain stable across age (e.g., self-esteem, Garisch \& Wilson, 2015). Future research could test this hypothesis by following a large cohort of adolescents across a number of years with NSSI measurements every couple of months.

\section{Strengths}

A key strength of this thesis is the use of longitudinal data to describe (Study Two) and begin to explain (Study Three) the developmental relationship between emotion 
regulation and NSSI. This is particularly important given that emotion regulation plays a key role in theoretical conceptualisations (Chapman et al., 2006; Hasking et al., 2016; Nock, 2009) and treatment (Gratz et al., 2012) of NSSI. Given the relative dearth of longitudinal NSSI research, particularly with adolescent samples (Fox et al., 2015), another key strength of this thesis is its use of three waves of longitudinal data which allows for greater precision than previous research with only two waves (Lundh et al., 2013; Lundh et al., 2011; Martin, et al., 2015; Prinstein et al., 2010; Tatnell et al., 2014). Critically, this thesis adds to the preliminary literature base which examines the integration of both intrapersonal and interpersonal risk factors for NSSI (see for example, Tatnell et al., 2014; You et al., 2012).

\section{Limitations}

A limitation of the current research is the use of a NSSI screening question. Several meta-analyses suggest that NSSI measurement type heavily influences NSSI prevalence (Muehlenkamp et al., 2012; Swannell et al., 2014) as well as effect sizes (Fox et al., 2015), with continuous measures (e.g., the DSHI-s) outperforming binary measures (e.g., lifetime history). Checklists are thought to provide a more accurate measurement because they rely on recognition memory, rather than free recall and so are less cognitively demanding (Schaeffer $\&$ Presser, 2003). I also argue that using a binary measure introduces the opportunity for bias, particularly for a socially-stigmatised behaviour such as NSSI. That is participants may rely on their stereotypes for what 'counts' as NSSI when evaluating their behaviours, potentially leading to under-reporting. Although the current studies included a checklist of NSSI behaviours (DSHI-s), this measure was only completed by participants who indicated on the NSSI screening question that they had thought about or engaged in NSSI, a binary measure. This branching procedure was put into place following concerns raised by the ethics committee regarding iatrogenic risk. In an attempt to mitigate the under-reporting, or a reliance on stereotypes of NSSI, the screening question included descriptions of a range of NSSI behaviours ("cut, burn, scratch, or carve their skin, bang or hit themselves, or prevent wounds from healing", see Appendix C). Within the current studies, NSSI prevalence rates are similar to international prevalence rates (Muehlenkamp et al., 2012; Swannell et al., 2014), and the effect size of NSSI status on emotion dysregulation (Study One) is similar to those reported in previous studies using checklist measures of NSSI (Eichen et al., 2015; Muehlenkamp et al., 2010; Perez et al., 2012). Together this replication suggests that the novel findings within this thesis are unlikely to be a result of measurement artifacts. However, future research should replicate this thesis' novel findings in other adolescent 
samples which assess NSSI using checklist measures. More broadly, the field as a whole would benefit from moving towards a standardised method of assessment in order limit the between-study heterogeneity associated with measurement type.

Another key limitation of this thesis is the narrow focus on emotion regulation, NSSI, and relationship attachments. Research investigating the development of NSSI is in its infancy, and at such early stages it is critical to first understand the foundational relationships. Replication of the reciprocal relationship between emotion regulation and NSSI with other longitudinal adolescent samples is needed. It is important to note Study Two and Study Three used alternative approaches to analysing the same data set to test complementary hypotheses, and therefore are not replications. Research replicating the bidirectional relationship between emotion regulation and NSSI may then expand to examine potential mediators and moderators of the relationship in order to test more specified models. Factors such as selfesteem (Garisch \& Wilson, 2015), depression (Marshall et al., 2013), identity confusion (Gandhi et al., 2017), sleep hygiene (Lundh et al., 2013), and peer NSSI (Prinstein et al., 2010), have been shown to play a role in the development of NSSI and may be sensible areas of inquiry. In addition, systematic tests to rule out potential third variables explaining this reciprocal relationship, such as environmental stressors, is necessary.

However, it is also critical for the field to develop a better understanding of NSSI desistence. The reciprocal relationship between emotion regulation and NSSI suggests that once a young person begins engaging in NSSI their increasing vulnerability creates a downward spiral of ever-increasing NSSI. However this prediction is contradicted by longitudinal research which finds that, although some people will continue to engage in NSSI during adulthood, for most people engagement in NSSI begins to decrease from age 16 onwards (Plener et al., 2015). One obvious possibility for this inconsistency between prediction and observation is that Study Two's longitudinal model does not include resiliency factors which may buffer against increased vulnerability. Another possibility is that as a young person ages, other equally effective (but maladaptive) emotion regulation strategies become available to them (e.g., alcohol, cannabis and other drugs), and so driven by the negative consequences of engaging in NSSI (e.g. extensive scaring, social stigma) they move on to other maladaptive emotion regulation behaviours (see Nock, 2009 for a similar argument). An empirically based, comprehensive model of NSSI onset, maintenance, and desistance could be built to simultaneously include multiple risk and preventative factors previously identified within the literature. Study Two and Study Three suggest that it may be 
necessary to take into account the potential for dynamic and reciprocal relationships between psychological predictors.

Another limitation of the current studies is that they rely on a very broad self-report measure of emotion regulation. Given the small number of items in each factor and the small amount of item cross-loading, Study One cautioned against using the three factors of the ERICA as distinct subscales. The ERICA then does not allow the research to distinguish between different facets of emotion regulation, such as reappraisal processes or emotional acceptance. Perhaps due to its origin as an observational measurement tool (MacDermott et al., 2010), the ERICA focuses on the behavioural consequences of failing to regulate emotion (e.g. "When things don't go my way I get upset easily"). Other items appear further removed from direct regulation processes by asking participants to make a judgement about their overall temperament (e.g. "I am a happy person"). However, given that the ERICA shows strong associations with the DERS, as well as similar relationships with aspects of wellbeing and NSSI, it seems reasonable that the ERICA is broadly capturing emotion regulation ability. Unfortunately, relying only on a broad view of emotion regulation prevents the differentiation of facets of emotion regulation such as emotional reactivity or emotional awareness, factors which the Cognitive-Emotional Model (Hasking et al., 2016), the Integrated Theoretical Model (Nock, 2009) and the Experiential Avoidance Model (Chapman et al., 2006) all suggest may play different though interconnected roles in the development and maintenance of NSSI. Future research can build a more nuanced understanding of the reciprocal relationship by delineating which aspects of emotion regulation, such as emotion reactivity, emotional acceptance, and use of specific strategies such as reappraisal and suppression are uniquely related to NSSI in order to create specific treatment targets.

\section{Future Directions}

This over-generalised focus is symptomatic of the field as a whole, insofar as the vast majority of work on the relationship between emotion regulation and NSSI has concentrated on subjective, self-reported trait emotion regulation and largely ignores the physiological aspects of emotional reactivity and regulation. Although an individual's evaluation of their trait emotion regulation abilities is critical for understanding the emotional response of people who self-injure, self-report measures are likely to contain biases in interpretation, memory, and report. These biases then make it difficult to isolate the specific deficits in emotion regulation which place an individual at risk of NSSI. For instance, previous research has established that people who self-injure report systematically lower emotion regulation than 
do controls (see for example, Eichen et al., 2015; Martin et al., 2010; Muehlenkamp et al., 2010), and Study One suggests that these deficiencies may already be present among adolescents thinking about self-injuring. However, it is unclear if this group difference is due to irregularities in the physiological generation of an emotional response, or in the subjective interpretation of that response. That is, adolescents who self-injure may have stronger emotional responses than those who don't, or they may have similar responses, but interpret them as stronger. This differentiation is also of clinical importance, as the strategies used to alter the physiological generation of emotion (e.g., deep breathing to reduce heart rate) differ from the strategies that alter the subjective interpretation of that emotional response (e.g., acceptance-based therapies, Hayes et al., 1999). Future research could begin to untangle this question by comparing the subjective and physiological responding of people who self-injure and controls during a laboratory emotional challenge manipulation, or by integrating wearable devices tracking physiological measurements (e.g., heart rate) alongside ecological momentary assessment study designs. Preliminary research has begun to investigate the psychophysiology and neurobiology of people who self-injure (see for example, Davis et al., 2014; Groschwitz, Plener, Groen, Bonenberger, \& Abler, 2016; Nock \& Mendes, 2008; Plener, Bubalo, Fladung, Ludolph, \& Lulé, 2012). These studies provide a useful starting point for further research to disentangle the complex relationship between emotion regulation and NSSI.

\section{Conclusions}

This thesis assessed the development of emotion regulation and NSSI during adolescence. Consistent with theoretical accounts of self-injury (Chapman et al., 2006; Hasking et al., 2016; Nock, 2010) as well as previous research (Fox et al., 2015), poor emotion regulation was established as a risk factor for self-injury a year later. However, selfinjury also predicted poorer emotion regulation a year later, demonstrating a reciprocal relationship of risk. Consistent with wider developmental research emphasising the primacy of social relationships for wellbeing, decreased attachment to parents and peers explained how self-injury affected subsequent emotion regulation. This thesis is the first to empirically demonstrate a reciprocal relationship between emotion regulation and self-injury and paves the way for future investigation into the dynamic relationship between psychological risk and protective factors and NSSI. More broadly, this thesis demonstrates that the strategies an adolescent uses to help them navigate their emotional world may have potentially profound effects on development. 


\section{References}

Abela, J. R. Z., Hankin, B. L., Haigh, E. A. P., Adams, P., Vinokuroff, T., \& Trayhern, L. (2005). Adolescent peer relations, friendships, and romantic relationships: Do they predict social anxiety and depression? Journal of Clinical Child and Adolescent Psychology, 34(1), 182-192.doi:10.1207/s15374424jccp3401_5

Adler, P. A., \& Adler, P. (2007). The demedicalization of self-injury from psychopathology to sociological deviance. Journal of Contemporary Ethnography, 36(5), 537-570. doi:10.1177/0891241607301968

Adrian, M., Zeman, J., Erdley, C., Lisa, L., \& Sim, L. (2011). Emotional dysregulation and interpersonal difficulties as risk factors for nonsuicidal self-injury in adolescent girls. Journal of Abnormal Child Psychology, 39(3), 389-400. doi:10.1007/s10802-010-94653

Ainsworth, M. D. S. (1969). Object relations, dependency, and attachment: A theoretical review of the infant-mother relationship. Child development, 969-1025. doi: $10.2307 / 1127008$

Aldao, A., Nolen-Hoeksema, S., \& Schweizer, S. (2010). Emotion-regulation strategies across psychopathology: A meta-analytic review. Clinical Psychology Review, 30(2), 217-237. doi:10.1016/j.cpr.2009.11.004

Andover, M. S., Primack, J. M., Gibb, B. E., \& Pepper, C. M. (2010). An examination of non-suicidal self-injury in men: Do men differ from women in basic NSSI characteristics? Archives of Suicide Research, 14(1), 79-88. doi:10.1080/13811110903479086

Andrews, T., Martin, G., Hasking, P., \& Page, A. (2014). Predictors of onset for non-suicidal self-injury within a school-based sample of adolescents. Prevention Science, 15(6), 850859. doi:10.1007/s11121-013-0412-8

Arbuckle, J. L. (2014). IMB SPSS Amos 23 user's guide. Crawfordville, FL: Amos Development Corporation.

Armsden, G. C., \& Greenberg, M. T. (1987). The inventory of parent and peer attachment: Individual differences and their relationship to psychological well-being in adolescence. Journal of Youth and Adolescence, 16(5), 427-454. doi:10.1007/BF02202939

Baetens, I., Claes, L., Martin, G., Onghena, P., Grietens, H., Van Leeuwen, K., ... Griffith, J. W. (2014). Is nonsuicidal self-injury associated with parenting and family factors? The Journal of Early Adolescence, 34(3), 387-405. doi:10.1177/0272431613494006

Bagley, C., \& Mallick, K. (2001). Normative data and mental health construct validity for the 
Rosenberg Self-Esteem Scale in British adolescents. International Journal of Adolescence and Youth, 9(2-3), 117-126. doi:10.1080/02673843.2001.9747871

Baumrind, D. (1987). A developmental perspective on adolescent risk taking in contemporary America. New directions for child and adolescent development, 37, 93-125. doi: $10.1002 / \mathrm{cd} .23219873706$

Bendelow, G., \& Williams, S. J. (1998). Emotions in social life: Critical themes and contemporary issues. London: Routledge.

Benson, M, J., McWey, L. M., \& Ross, J. J. (2006). Parental attachment and peer relations in adolescence: A meta-analysis. Research in Human Development, 3(1), 33-43. doi: 10.1207/s15427617rhd0301_4

Bergman, L. R., \& Magnusson, D. (1997). A person-oriented approach in research on developmental psychopathology. Development and Psychopathology, 9(2), 291-319. doi:10.1017/S095457949700206X

Bildik, T., Somer, O., Kabukçu Başay, B., Başay, Ö., \& Özbaran, B. (2013). The validity and reliability of the Turkish version of the Inventory of Statements About Self-injury. Turkish Journal of Psychiatry, 24(1), 41-49. doi:10.5080/u6901

Bjärehed, J., \& Lundh, L.-G. (2008). Deliberate self-harm in 14-year-old adolescents: How frequent is it, and how is it associated with psychopathology, relationship variables, and styles of emotional regulation? Cognitive Behaviour Therapy, 37(1), 26-37. doi:10.1080/16506070701778951

Bowlby, J. (1982) Attachment and loss: Vol. 1. Attachment. New York, NY: Basic Books. Bradley, M. M., \& Lang, P. J. (2007). Emotion and motivation. In Cacioppo, J. T., Tassinary, L. G., \& Berntson, G. (Eds.). Handbook of psychophysiology, (pp. 602-642). New York, NY: Cambridge University Press.

Bradley, M. M., \& Lang, P. J. (1994). Measuring emotion: The self-assessment manikin and the semantic differential. Journal of Behavior Therapy and Experimental Psychiatry, 25(1), 49-59. doi:10.1016/0005-7916(94)90063-9

Brechwald, W. A., \& Prinstein, M. J. (2011). Beyond homophily: A decade of advances in understanding peer influence processes. Journal of Research on Adolsecence, 21(1), 166-179. doi:10.1111/j.1532-7795.2010.00721.x

Brown, M. Z., Comtois, K. A., \& Linehan, M. M. (2002). Reasons for suicide attempts and nonsuicidal self-injury in women with borderline personality disorder. Journal of Abnormal Psychology, 111(1), 198-202. doi:10.1037/0021-843X.111.1.198

Bunford, N., Evans, S. W., Becker, S. P., \& Langberg, J. M. (2015). Attention- 
Deficit/Hyperactivity Disorder and social skills in youth: A moderated mediation model of emotion dysregulation and depression. Journal of Abnormal Child Psychology, 43(2), 283-296. doi:10.1007/s10802-014-9909-2

Bunford, N., Evans, S. W., \& Langberg, J. M. (2014). Emotion dysregulation is associated with social impairment among young adolescents with ADHD. Journal of Attention Disorders. doi:10.1177/1087054714527793

Bunford, N., Evans, S. W., \& Wymbs, F. (2015). ADHD and emotion dysregulation among children and adolescents. Clinical Child and Family Psychology Review, 18(3). doi:10.1007/s10567-015-0187-5

Burke, T. A., Hamilton, J. L., Abramson, L. Y., \& Alloy, L. B. (2015). Non-suicidal selfinjury prospectively predicts interpersonal stressful life events and depressive symptoms among adolescent girls. Psychiatry Research, 228(3), 416-424. doi:10.1016/j.psychres.2015.06.021

Butler, A. M., \& Malone, K. (2013). Attempted suicide v. non-suicidal self-injury: Behaviour syndrome or diagnosis? British Journal of Psychiatry, 202(5), 324-325. doi:10.1192/bjp.bp.112.113506

Byrne, S., Morgan, S., Fitzpatrick, C., Boylan, C., Crowley, S., Gahan, H., ... Guerin, S. (2008). Deliberate self-harm in children and adolescents: A qualitative study exploring the needs of parents and carers. Clinical Child Psychology and Psychiatry, 13(4), 493504. doi:10.1177/1359104508096765

Calkins, S. D., \& Hill, A. (2007). Caregiver influences on emerging emotion regulation. In J. J. Gross (Ed.), Handbook of emotion regulation, (pp. 229-248). New York, NY: Guilford Press.

Cattell, R. B. (1966). The scree test for the number of factors. Multivariate Behavioral Research, 1(2), 245-276. doi:10.1207/s15327906mbr0102_10

Chan, D. (1998). The conceptualization and analysis of change over time: An integrative ppproach incorporating Longitudinal Mean and Covariance Structures Analysis (LMACS) and Multiple Indicator Latent Growth Modeling (MLGM). Organizational Research Methods, 1(4), 421-483.

Chaplin, T. M., Cole, P. M., \& Zahn-Waxler, C. (2005). Parental socialization of emotion expression: Gender differences and relations to child adjustment. Emotion, 5(1), 80-88. doi:10.1037/1528-3542.5.1.80

Chapman, A. L., Gratz, K. L., \& Brown, M. Z. (2006). Solving the puzzle of deliberate selfharm: The experiential avoidance model. Behaviour Research and Therapy, 44(3), 371- 
394. doi:10.1016/j.brat.2005.03.005

Claes, L., Houben, A., Vandereycken, W., Bijttebier, P., \& Muehlenkamp, J. (2010). Brief report: The association between non-suicidal self-injury, self-concept and acquaintance with self-injurious peers in a sample of adolescents. Journal of Adolescence, 33(5), 775-778. doi:10.1016/j.adolescence.2009.10.012

Cohen, J. M. (1977). Sources of peer group homogeneity. Sociology of Education, 50(4), 227-241. doi:10.2307/2112497

Cole, P. M., Michel, M. K., \& Teti, L. O. (1994). The development of emotion regulation and dysregulation: A clinical perspective. Monographs of the Society for Research in Child Development, 59(2/3), 73-100. doi:10.2307/1166139

Cox, L. J., Stanley, B. H., Melhem, N. M., Oquendo, M. A., Birmaher, B., Burke, A., ... Brent, D. A. (2012). A longitudinal study of nonsuicidal self-injury in offspring at high risk for mood disorder. Journal of Clinical Psychiatry, 73(6), 821-828. doi:10.4088/JCP.11m07250

Cox, M. J., \& Paley, B. (2003). Understanding families as systems. Current Directions in Psychological Science, 12(5), 193-196. doi:10.1111/1467-8721.01259

Davidson, R. J. (1998). Affective style and affective disorders: Perspectives from affective neuroscience. Cognition \& Emotion, 12(3), 307-330. doi:10.1080/026999398379628

Davis, T. S., Mauss, I. B., Lumian, D., Troy, A. S., Shallcross, A. J., Zarolia, P., .. McRae, K. (2014). Emotional reactivity and emotion regulation among adults with a history of self-harm: Laboratory self-report and functional MRI evidence. Journal of Abnormal Psychology, 123(3), 499-509. doi:10.1037/a0036962

Di Pierro, R., Sarno, I., Perego, S., Gallucci, M., \& Madeddu, F. (2012). Adolescent nonsuicidal self-injury: The effects of personality traits, family relationships and maltreatment on the presence and severity of behaviours. European Child and Adolescent Psychiatry, 21(9), 511-520. doi:10.1007/s00787-012-0289-2

Duggan, J., Heath, N., \& Hu, T. (2015). Non-suicidal self-injury maintenance and cessation among adolescents: a one-year longitudinal investigation of the role of objectified body consciousness, depression and emotion dysregulation. Child and Adolescent Psychiatry and Mental Health, 9:21. doi:10.1186/s13034-015-0052-9

Edmondson, A. J., Brennan, C. A., \& House, A. O. (2016). Non-suicidal reasons for selfharm: A systematic review of self-reported accounts. Journal of Affective Disorders, 191, 109-117. doi:10.1016/j.jad.2015.11.043

Eichen, D. M., Kass, A. E., Fitzsimmons-Craft, E. E., Gibbs, E., Trockel, M., Barr Taylor, C., 
\& Wilfley, D. E. (2015). Non-suicidal self-injury and suicidal ideation in relation to eating and general psychopathology among college-age women. Psychiatry Research, 235, 77-82. doi:10.1016/j.psychres.2015.11.046

Eisenberg, N., Cumberland, A., Spinrad, T. L., Fabes, R. A., Shepard, S. A., Reiser, M., ... Guthrie, I. K. (2001). The relations of regulation and emotionality to children's externalizing and internalizing problem behavior. Child Development, 72(4), 11121134.

Eisenberger, N. I., \& Lieberman, M. D. (2004). Why rejection hurts: A common neural alarm system for physical and social pain. Trends in Cognitive Sciences, 8(7), 294-300. doi:10.1016/j.tics.2004.05.010

Ekman, P. (1992). An argument for basic emotions. Cognition \& Emotion, 6(3-4). 169-200.

Engels, R. C. M. E., Finkenauer, C., Meeus, W., \& Deković, M. (2001). Parental attachment and adolescent's emotional adjustment: The associations with social skills and relational competence. Journal of Counseling Psychology, 428-439. doi:10.1037/00220167.48.4.428

Favazza, A. R., \& Conterio, K. (1988). The plight of chronic self-mutilators. Community Mental Health Journal, 24(1), 22-30. doi:10.1007/BF00755050

Finch, J. F., Okun, M. A, Pool, G. J., \& Ruehlman, L. S. (1999). A comparison of the influence of conflictual and supportive social interactions on psychological distress. Journal of Personality, 67, 581-621. doi:10.1111/1467-6494.00066

Fortune, S., Sinclair, J., \& Hawton, K. (2008). Help-seeking before and after episodes of selfharm: A descriptive study in school pupils in England. BMC Public Health, 8:369. doi:10.1186/1471-2458-8-369

Fox, K. R., Franklin, J. C., Ribeiro, J. D., Kleiman, E. M., Bentley, K. H., \& Nock, M. K. (2015). Meta-analysis of risk factors for nonsuicidal self-injury. Clinical Psychology Review, 42, 156-167. doi:10.1016/j.cpr.2015.09.002

Franklin, J. C., Hessel, E. T., Aaron, R. V, Arthur, M. S., Heilbron, N., \& Prinstein, M. J. (2010). The functions of nonsuicidal self-injury: Support for cognitive-affective regulation and opponent processes from a novel psychophysiological paradigm. Journal of Abnormal Psychology, 119(4), 850-862. doi:10.1037/a0020896

Gandhi, A., Luyckx, K., Maitra, S., Kiekens, G., Verschueren, M., \& Claes, L. (2017).

Directionality of effects between non-suicidal self-injury and identity formation: A prospective study in adolescents. Personality and Individual Differences, 109, 124-129. doi:10.1016/j.paid.2017.01.003 
Garisch, J. A., \& Wilson, M. S. (2015). Prevalence, correlates, and prospective predictors of non-suicidal self-injury among New Zealand adolescents: Cross-sectional and longitudinal survey data. Child and Adolescent Psychiatry and Mental Health, 9:28. doi:10.1186/s13034-015-0055-6

Garnefski, N., Kraaij, V., \& Spinhoven, P. (2001). Negative life events, cognitive emotion regulation and emotional problems. Personality and Individual Differences, 30(8), 1311-1327. doi:10.1016/S0191-8869(00)00113-6

Garofalo, C., Holden, C. J., Zeigler-Hill, V., \& Velotti, P. (2016). Understanding the connection between self-esteem and aggression: The mediating role of emotion dysregulation. Aggressive Behavior, 42(1), 3-15. doi:10.1002/ab.21601

Gibb, S. J., Beautrais, A. L., \& Surgenor, L. J. (2010). Health-care staff attitudes towards self-harm patients. The Australian and New Zealand Journal of Psychiatry, 44(8), 71320. doi:10.3109/00048671003671015

Giles, D. (2013). Advanced research methods in psychology. New York, NY: Routledge.

Giletta, M., Scholte, R. H. ., Engels, R. C. M. E., Ciairano, S., \& Prinstein, M. J. (2012). Adolescent non-suicidal self-injury: A cross-national study of community samples from Italy, the Netherlands and the United States. Psychiatry Research, 197(1-2), 66-72. doi:10.1016/j.psychres.2012.02.009.

Gorrese, A., \& Ruggieri, R. (2012). Peer attachment: A meta-analytic review of gender and age differences and associations with parent attachment. Journal of Youth and Adolescence, 41(5), 650-672. doi:10.1007/s10964-012-9759-6

Gratz, K. L. (2001). Measurement of deliberate self-harm: Preliminary data on the Deliberate Self-Harm Inventory. Journal of Psychopathology and Behavioral Assessment, 23(4), 253-263. doi:10.1023/A:1012779403943

Gratz, K. L. (2003). Risk factors for and functions of deliberate self-harm: An empirical and conceptual review. Clinical Psychology: Science and Practice, 10(2), 192-205. doi:10.1093/clipsy/bpg022

Gratz, K. L., Breetz, A., \& Tull, M. T. (2010). The moderating role of borderline personality in the relationships between deliberate self-harm and emotion-related factors.

Personality and Mental Health, 4(2), 96-107. doi:10.1002/pmh.102

Gratz, K. L., \& Gunderson, J. G. (2006). Preliminary data on an acceptance-based emotion regulation group intervention for deliberate self-harm among women with borderline Personality Disorder. Behavior Therapy, 37(1), 25-35. doi:10.1016/j.beth.2005.03.002

Gratz, K. L., Levy, R., \& Tull, M. T. (2012). Emotion regulation as a mechanism of change 
in an acceptance-based emotion regulation group therapy for deliberate self-harm among women with borderline personality pathology. Journal of Cognitive Psychotherapy: An International Quarterly, 26(4), 365-381. doi:10.1891/0889-8391.26.4.365

Gratz, K. L., \& Roemer, L. (2004). Multidimensional assessment of emotion regulation and dysregulation: Development, factor structure, and initial validation of the Difficulties in Emotion Regulation Scale. Journal of Psychopathology and Behavioral Assessment, 26(1), 41-54. doi:10.1023/B:JOBA.0000007455.08539.94

Gratz, K. L., \& Tull, M. T. (2010). The relationship between emotion dysregulation and deliberate self-harm among inpatients with substance use disorders. Cognitive Therapy and Research, 34(6), 544-553. doi:10.1007/s10608-009-9268-4

Gratz, K. L., \& Tull, M. T. (2011). Extending research on the utility of an adjunctive emotion regulation group therapy for deliberate self-harm among women with borderline personality pathology. Personality Disorders: Theory, Research, and Treatment, 2(4), 316-326. doi:10.1037/a0022144

Groschwitz, R. C., Plener, P. L., Groen, G., Bonenberger, M., \& Abler, B. (2016). Differential neural processing of social exclusion in adolescents with non-suicidal selfinjury: An fMRI study. Psychiatry Research: Neuroimaging, 255, 43-49. doi:10.1016/j.pscychresns.2016.08.001

Gross, J. J. (1998). The emerging field of emotion regulation: An integrative review. Review of General Psychology, 2(5), 271-299.

Gross, J. J. (2015). Emotion regulation: Current status and future prospects. Psychological Inquiry, 26(1), 1-26. doi:10.1080/1047840X.2014.940781

Gross, J. J., \& John, O. P. (2003). Individual differences in two emotion regulation processes: Implications for affect, relationships, and well-being. Journal of Personality and Social Psychology, 85(2), 348-362. doi:10.1037/0022-3514.85.2.348

Gross, J. J., \& Thompson, R. A. (2007). Emotion regulation: Conceptual foundations. In J. J. Gross (Ed.), Handbook of emotion regulation, (pp. 3-24). New York, NY: Guilford Press.

Guan, K., Fox, K. R., \& Prinstein, M. J. (2012). Nonsuicidal self-injury as a time-invariant predictor of adolescent suicide ideation and attempts in a diverse community sample. Journal of Consulting and Clinical Psychology, 80(5), 842-849. doi:10.1037/a0029429

Guerry, J. D., \& Prinstein, M. J. (2009). Longitudinal prediction of adolescent nonsuicidal self-injury: Examination of a cognitive vulnerability-stress model. Journal of Clinical Child \& Adolescent Psychology, 39, 77-89. doi:10.1080/15374410903401195 
Hankin, B. L., \& Abela, J. R. Z. (2011). Nonsuicidal self-injury in adolescence: Prospective rates and risk factors in a $2 \frac{1}{2} 2$ year longitudinal study. Psychiatry Research, 186(1), 6570. doi:10.1016/j.psychres.2010.07.056

Hariri, A. R., \& Holmes, A. (2006). Genetics of emotional regulation: The role of the serotonin transporter in neural function. Trends in cognitive sciences, 10(4), 182-191. doi: 10.1016/j.tics.2006.02.011

Harris, J. (2000). Self-harm: Cutting the bad out of me. Qualitative Health Research, 10(2), 164-173. doi:10.1177/104973200129118345

Hasking, P. A., Coric, S. J., Swannell, S., Martin, G., Thompson, H. K., \& Frost, A. D. J. (2010). Brief report: Emotion regulation and coping as moderators in the relationship between personality and self-injury. Journal of Adolescence, 33(5), 767-773. doi:10.1016/j.adolescence.2009.12.006

Hasking, P., Andrews, T., \& Martin, G. (2013). The role of exposure to self-injury among peers in predicting later self-injury. Journal of Youth and Adolescence, 42(10), $1543-$ 1556. doi:10.1007/s10964-013-9931-7

Hasking, P., Whitlock, J., Voon, D., \& Rose, A. (2016). A cognitive-emotional model of NSSI: Using emotion regulation and cognitive processes to explain why people selfinjure. Cognition and Emotion. doi:10.1080/02699931.2016.1241219

Hawton, K., Rodham, K., Evans, E., \& Weatherall, R. (2002). Deliberate self harm in adolescents: Self report survey in schools in England. British Medical Journal, 325(7374), 1207-1211. doi:10.1136/bmj.325.7374.1207

Hawton, K., Rodham, K., \& Evans, E. (2006). By their own young hand. Deliberate selfharm and suicidal ideas in adolescents. London: Jessica Kingsley Publishing.

Hawton, K., Saunders, K. E. A., \& O'Connor, R. C. (2012). Self-harm and suicide in adolescents. The Lancet, 379(9834), 2373-2382. doi:10.1016/S0140-6736(12)60322-5

Hayes, A. F. (2012). PROCESS: A versatile computational tool for observed variable mediation, moderation, and conditional process modeling. Retrieved from http://www.processmacro.org/index.html

Hayes, S. C., Strosahl, K. D., \& Wilson, K. G. (1999). Acceptance and commitment therapy: An experiential approach to behavior change. New York, NY: Guilford Press.

Heath, N. L., Toste, J. R., \& Beettam, E. L. (2007). “ I am not well-equipped ”: High school teachers's perceoptions of self-injury. Canadian Journal of School Psychology, 21(1/2), 73-92. doi: 10.1177/0829573506298471

Henry, J. D., \& Crawford, J. R. (2005). The short-form version of the Depression Anxiety 
Stress Scales (DASS-21): Construct validity and normative data in a large non-clinical sample. The British Journal of Clinical Psychology, 44(2), 227-39. doi:10.1348/014466505X29657

Herzog, T. K., Hill-Chapman, C., Hardy, T. K., Wrighten, S. A., \& El-Khabbaz, R. (2015). Trait emotion, emotional regulation, and parenting styles. Journal of Educational and Developmental Psychology, 5(2), 119-135. doi:10.5539/jedp.v5n2p119

Hilt, L. M., Nock, M. K., Lloyd-Richardson, E. E., \& Prinstein, M. J. (2008). Longitudinal study of nonsuicidal self-injury among young adolescents: Rates, correlates, and preliminary test of an interpersonal model. Journal of Early Adolescence, 28(3), 455469. doi:10.1177/0272431608316604

Horn, J. L. (1965). A rationale and test for the number of factors in factor analysis. Psychometrika, 30(2), 179-185. doi:10.1007/BF02289447

Hughes, E. K., Gullone, E., \& Watson, S. D. (2011). Emotional functioning in children and adolescents with elevated depressive symptoms. Journal of Psychopathology and Behavioral Assessment, 33(3), 335-345. doi:10.1007/s10862-011-9220-2

Ijzendoorn, M. H. Van, \& Kroonenberg, P. M. (1988). Cross-cultural patterns of attachment: A meta-analysis of the strange situation, 59(1), 147-156. doi:10.2307/1130396 International Society for the Study of Self-Injury. (2016). Fast facts. Retrieved from: http://itriples.org/redesadmin15/fast-facts/

Kaiser, H. F. (1974). An index of factorial simplicity. Psychometrika, 39(1), 31-36. doi:10.1007/BF02291575

Kenny, D. A. (1975). Cross-lagged panel correlation: A test for spuriousness. Psychological Bulletin, 82(6), 887-903. doi:10.1037/0033-2909.82.6.887

Kenny, D. A. (2005). Cross-Lagged Panel Design. Wiley StatsRef: Statistics Reference Online. doi:10.1002/9781118445112.stat06464

Klineberg, E., Stansfeld, S. A., \& Bhui, K. S. (2013). How do adolescents talk about selfharm? Findings from a qualitative school-based study in England. Journal of Adolescent Health, 52(2), S78-S79. doi:10.1016/j.jadohealth.2012.10.183

Klonsky, E. D. (2007). The functions of deliberate self-injury: A review of the evidence. Clinical Psychology Review, 27(2), 226-239. doi:10.1016/j.cpr.2006.08.002

Klonsky, E. D. (2011). Non-suicidal self-injury in United States adults: Prevalence, sociodemographics, topography and functions. Psychological Medicine, 41(9), 19811986. doi: $10.1017 / \mathrm{S} 0033291710002497$

Klonsky, E. D., \& Glenn, C. R. (2009). Assessing the functions of non-suicidal self-injury: 
Psychometric properties of the Inventory of Statements about Self-injury (ISAS). Journal of Psychopathology and Behavioral Assessment, 31:215. doi:10.1007/s10862008-9107-z

Klonsky, E. D., \& Olino, T. M. (2008). Identifying clinically distinct subgroups of selfinjurers among young adults: A latent class analysis. Journal of Consulting and Clinical Psychology, 76(1), 22-27. doi:10.1037/0022-006X.76.1.22

Knapp, M., \& McDaid, D. (2009). Making an economic case for prevention and promotion. International Journal of Mental Health Promotion, 11(3), 49-56. doi:10.1080/14623730.2009.9721792

Koenig, J., Rinnewitz, L., Niederbäumer, M., Strozyk, T., Parzer, P., Resch, F., \& Kaess, M. (2017). Longitudinal development of pain sensitivity in adolescent non-suicidal selfinjury. Journal of Psychiatric Research, 89, 81-84. doi:10.1016/j.jpsychires.2017.02.001

Kortge, R., Meade, T., \& Tennant, A. (2013). Interpersonal and intrapersonal functions of Deliberate Self-Harm (DSH): A psychometric examination of the Inventory of Statements About Self-Injury (ISAS) scale. Behaviour Change, 30(1), 24-35. doi:10.1017/bec.2013.3

Kraemer H. C, Kazdin, A. E,. Offord D. R, Kessler R. C, Jensen P. S, \& Kupfer, D. J. (1997). Coming to terms with the terms of risk. Archives of General Psychiatry, 54(4), 337-343. doi:10.1001/archpsyc.1997.01830160065009

Laible, D. J. J., Carlo, G., \& Raffaelli, M. (2000). The differential relations of parent and peer attachment to adolescent adjustment. Journal of Youth and Adolescence, 29(1), 45-59.

Langlands, R. L. (2012). Does non-suicidal self-injury function primarily as an experientially avoidant behaviour within Aotearoa New Zealand? (Unpublished doctoral dissertation) Victoria University of Wellington: Wellington, New Zealand.

Lane, R. D., \& Pollermann, B. Z. (2002). Complexity of emotion representations. In: L. F. Barrett, \& P. Salovey (Eds.), The wisdom in feeling: Psychological processes in emotional intelligence. New York, NY: Guilford Press.

Laye-Gindhu, A., \& Schonert-Reichl, K. A. (2005). Nonsuicidal self-harm among community adolescents: Understanding the "whats" and "whys" of self-harm. Journal of Youth and Adolescence, 34(5), 447-457. doi:10.1007/s10964-005-7262-z

Lazarus, R. S., \& Alfert, E. (1964). Short-circuiting of threat by experimentally altering cognitive appraisal. Journal of Abnormal and Social Psychology, 69(2), 195-205. doi: $10.1037 / \mathrm{h} 0044635$ 
Lee, I. A., \& Preacher, K. J. (2013, September). Calculation for the test of the difference between two dependent correlations with one variable in common [Computer software]. Available from http://quantpsy.org.

Linehan, M. (1993). Cognitive-behavioral treatment of borderline personality disorder. New York: Guilford Press.

Lundh, L. G., Bjärehed, J., \& Wångby-Lundh, M. (2013). Poor sleep as a risk factor for nonsuicidal self-injury in adolescent girls. Journal of Psychopathology and Behavioral Assessment, 35(1), 85-92. doi:10.1007/s10862-012-9307-4

Lundh, L. G., Wångby-Lundh, M., \& Bjärehed, J. (2011). Deliberate self-harm and psychological problems in young adolescents: Evidence of a bidirectional relationship in girls. Scandinavian Journal of Psychology, 52(5), 476-483. doi:10.1111/j.14679450.2011.00894.x

Lundh, L., Karim, J., \& Quilisch, E. V. a. (2007). Development and aging deliberate selfharm in 15-year-old adolescents: A pilot study with a modified version of the Deliberate Self-Harm Inventory. Scandinavian Journal of Psychology, (1997), 33-41. doi:10.1111/j.1467-9450.2006.00567.x

Luppa, M., Heinrich, S., Angermeyer, M. C., König, H.-H., \& Riedel-Heller, S. G. (2007). Cost-of-illness studies of depression: A systematic review. Journal of Affective Disorders, 98(1-2), 29-43. doi:10.1016/j.jad.2006.07.017

MacDermott, S. T., Gullone, E., Allen, J. S., King, N. J., \& Tonge, B. (2010). The Emotion Regulation Index for Children and Adolescents (ERICA): A psychometric investigation. Journal of Psychopathology and Behavioral Assessment, 32(3), 301-314. doi:10.1007/s10862-009-9154-0

Marshall, S. K., Tilton-Weaver, L. C., \& Stattin, H. (2013). Non-suicidal self-injury and depressive symptoms during middle adolescence: A longitudinal analysis. Journal of Youth and Adolescence, 42(8), 1234-1242. doi:10.1007/s10964-013-9919-3

Martin, G., Swannell, S. V., Hazell, P. L., Harrison, J. E., \& Taylor, A. W. (2010). Self-injury in Australia: A community survey. Medical Journal of Australia, 193(9), 506-510.

Martin, J., Bureau, J. F., Cloutier, P., \& Lafontaine, M. F. (2011). A comparison of invalidating family environment characteristics between university students engaging in self-injurious thoughts \& actions and non-self-injuring university students. Journal of Youth and Adolescence, 40(11), 1477-1488. doi:10.1007/s10964-011-9643-9

Martin, J., Bureau, J.-F., Yurkowski, K., Fournier, T. R., Lafontaine, M.-F., \& Cloutier, P. (2016). Family-based risk factors for non-suicidal self-injury: Considering influences of 
maltreatment, adverse family-life experiences, and parent-child relational risk. Journal of Adolescence, 49, 170-180. doi:10.1016/j.adolescence.2016.03.015

Matsumoto, D., Yoo, S. H., \& Nakagawa, S. (2008). Culture, emotion regulation, and adjustment. Journal of Personality and Social Psychology, 94(6), 925-937. doi:10.1037/0022-3514.94.6.925

Mauss, I. B., Levenson, R. W., McCarter, L., Wilhelm, F. H., \& Gross, J. J. (2005). The tie that binds? Coherence among emotion experience, behavior, and physiology. Emotion, 5(2), 175-190. doi:10.1037/1528-3542.5.2.175

McAllister, M., Creedy, D., Moyle, W., \& Farrugia, C. (2002). Methodological issues in nursing research: Nurses' attitudes towards clients who self-harm. Journal of Advanced Nursing, 40(5), 578-586. doi:10.1046/j.1365-2648.2002.02412.x

McDonald, G., O’Brien, L., \& Jackson, D. (2007). Guilt and shame: Experiences of parents of self-harming adolescents. Journal of Child Health Care, 11(4), 298-310. doi:10.1177/1367493507082759

McHale, J., \& Felton, A. (2010). Self-harm: What's the problem? A literature review of the factors affecting attitudes towards self-harm. Journal of Psychiatric and Mental Health Nursing, 17(8), 732-740. doi:10.1111/j.1365-2850.2010.01600.x

McKenzie, K. C., \& Gross, J. J. (2014). Nonsuicidal self-injury: An emotion regulation perspective. Psychopathology, 47(4), 207-219. doi:10.1159/000358097

McRae, K., Gross, J. J., Weber, J., Robertson, E. R., Sokol-Hessner, P., Ray, R. Gabrielli, J. D. E., \& Ochsner, K. N. (2012). The development of emotion regulation: An fMRI study of cognitive reappraisal in children, adolescents and young adults. Social Cognitive and Affective Neuroscience, 7(1), 11-22. doi:10.1093/scan/nsr093

Moreno, C., Garcia-Moya, I., Rivera, F., \& Ramos, P. (2016). Characterization of vulnerable and resilient Spanish adolescents in their developmental contexts. Frontiers in Psychology, 7:983. doi:10.3389/fpsyg.2016.00983

Morris, A. S., Silk, J. S., Steinberg, L., Myers, S. S., \& Robinson, L. R. (2007). The role of the family context in the development of emotion regulation. Social Development, 16(2), 361-388. doi:10.1111/j.1467-9507.2007.00389.x

Muehlenkamp, J. J., Brausch, A., Quigley, K., \& Whitlock, J. (2013). Interpersonal features and functions of nonsuicidal self-injury. Suicide and Life-Threatening Behavior, 43(1), 67-80. doi:10.1111/j.1943-278X.2012.00128.x

Muehlenkamp, J. J., Claes, L., Havertape, L., \& Plener, P. L. (2012). International prevalence of adolescent non-suicidal self-injury and deliberate self-harm. Child and Adolescent 
Psychiatry and Mental Health, 6:10. doi:10.1186/1753-2000-6-10

Muehlenkamp, J. J., Claes, L., Smits, D., Peat, C. M., \& Vandereycken, W. (2011). Nonsuicidal self-injury in eating disordered patients: A test of a conceptual model. Psychiatry Research, 188(1), 102-108. doi:10.1016/j.psychres.2010.12.023

Muehlenkamp, J. J., Engel, S. G., Wadeson, A., CRosby, R. D., Wonderlich, S. A., Simonisch, H., \& Mitchell, J. E. (2009). Emotional states preceding and following acts of non-suicidal self-injury in bulimia nervosa patients. Behavior Research and Therapy, 47(1), 83-87. doi:10.1016/j.brat.2008.10.011

Muehlenkamp, J. J., Kerr, P. L., Bradley, A. R., \& Larsen, M. A. (2010). Abuse subtypes and nonsuicidal self-injury. The Journal of Nervous and Mental Disease, 198(4), 258-263. doi:10.1097/NMD.0b013e3181d612ab

Muehlenkamp, J. J., \& Kerr, P. L. (2010). Untangling a complex web: How non-suicidal selfinjury and suicide attempts differ. The Prevention Researcher, 17(1), 8-11.

Nada Raja, S., McGee, R., \& Stanton, W. R. (1992). Perceived attachments to parents and peers and psychological well-being in adolescence. Journal of Youth and Adolescence, 21(4), 471-485. doi:10.1007/BF01537898

Nixon, M. K., Cloutier, P., \& Jansson, S. M. (2008). Nonsuicidal self-harm in youth: A population-based survey. Canadian Medical Association Journal Journal, 178(3), 306312. doi:10.1503/cmaj.061693

Nock, M. K. (2009). Why do people hurt themselves? New insights into the nature and functions of self-injury. Current Directions in Psychological Science, 18(2), 78-83. doi:10.1111/j.1467-8721.2009.01613.x

Nock, M. K. (2010). Self-Injury. Annual Review of Clinical Psychology, 6(1), 339-363. doi:10.1146/annurev.clinpsy.121208.131258

Nock, M. K., \& Mendes, W. B. (2008). Physiological arousal, distress tolerance, and social problem-solving deficits among adolescent self-injurers. Journal of Consulting and Clinical Psychology, 76(1), 28-38. doi:10.1037/0022-006X.76.1.28

Nock, M. K., \& Prinstein, M. J. (2004). A Functional Approach to the Assessment of SelfMutilative Behavior. Journal of Consulting and Clinical Psychology, 72(5), 885-890. doi:10.1037/0022-006X.72.5.885

Nock, M. K., Prinstein, M. J., \& Sterba, S. K. (2009). Revealing the form and function of self-injurious thoughts and behaviors: A real-time ecological assessment study among adolescents and young adults. Journal of Abnormal Psychology, 118(4), 816-827. doi:10.1037/a0016948 
Norton, P. J. (2007). Depression Anxiety and Stress Scales (DASS-21): Psychometric analysis across four racial groups. Anxiety, Stress, and Coping, 20(3), 253-265. doi:10.1080/10615800701309279

Nunnally, J. C. (1978). Psychometric theory (2nd ed.). New York, NY: McGraw-Hill. O’Dea, D., \& Wren, J. (2010). New Zealand Estimates of the Total Social and Economic Cost of "All Injuries" and the Six Priority Areas Respectively, at June 2008 prices: Technical Report prepared for New Zealand Injury Prevention Strategy Evaluation. Wellington: University of Otago and Accident Compensation Corporation.

Park, C. L., Edmondson, D., \& Lee, J. (2012). Development of self-regulation abilities as predictors of psychological adjustment across the first year of college. Journal of Adult Development, 19(1), 40-49. doi:10.1007/s10804-011-9133-Z

Perez, J., Venta, A., Garnaat, S., \& Sharp, C. (2012). The Difficulties in Emotion Regulation Scale: Factor structure and association with nonsuicidal self-injury in adolescent inpatients. Journal of Psychopathology and Behavioral Assessment, 34(3), 393-404. doi:10.1007/s10862-012-9292-7

Phillips, K. F. V, \& Power, M. J. (2007). A new self-report measure of emotion regulation in adolescents: The Regulation of Emotions Questionnaire. Clinical Psychology and Psychotherapy, 14, 145-156. doi:10.1002/cpp.523

Plener, P. L., Bubalo, N., Fladung, A. K., Ludolph, A. G., \& Lulé, D. (2012). Prone to excitement: Adolescent females with Non-Suicidal Self-Injury (NSSI) show altered cortical pattern to emotional and NSS-related material. Psychiatry Research: Neuroimaging, 203(2-3), 146-152. doi:10.1016/j.pscychresns.2011.12.012

Plener, P. L., Libal, G., Keller, F., Fegert, J. M., \& Muehlenkamp, J. J. (2009). An international comparison of adolescent non-suicidal self-injury (NSSI) and suicide attempts: Germany and the USA. Psychological Medicine, 39(9), 1549-1558. doi:10.1017/S0033291708005114

Plener, P. L., Schumacher, T. S., Munz, L. M., \& Groschwitz, R. C. (2015). The longitudinal course of non-suicidal self-injury and deliberate self-harm: A systematic review of the literature. Borderline Personality Disorder and Emotion Dysregulation, 2:2. doi:10.1186/s40479-014-0024-3

Ployhart, R., \& Vandenberg, R. (2010). Longitudinal research: The theory, design, and analysis of change. Journal of Management, 36(1), 94-120. doi:10.1177/0149206309352110

Preacher, K. J., \& Hayes, A. F. (2008). Asymptotic and resampling strategies for assessing 
and comparing indirect effects in multiple mediator models. Behavior Research Methods, 40(3), 879-891. doi:10.3758/BRM.40.3.879

Prinstein, M. J. (2008). Introduction to the special section on suicide and nonsuicidal selfinjury: A review of unique challenges and important directions for self-injury science. Journal of Consulting and Clinical Psychology, 76(1), 1-8. doi:10.1037/0022006X.76.1.1

Prinstein, M. J., Heilbron, N., Guerry, J. D., Franklin, J. C., Rancourt, D., Simon, V., \& Spirito, A. (2010). Peer influence and nonsuicidal self injury: Longitudinal results in community and clinically-referred adolescent samples. Journal of Abnormal Child Psychology, 38(5), 669-682. doi:10.1007/s10802-010-9423-0

Raver, C. C. (2004). Placing emotional self-regulation in sociocultural and socioeconomic contexts. Child Development, 75(2), 346-353.

Rosenberg, M. (1965). Society and the adolescent self-image. Princeton, NJ: Princeton University Press.

Salters-Pedneault, K., Tull, M. T., \& Roemer, L. (2004). The role of avoidance of emotional material in the anxiety disorders. Applied and Preventive Psychology, 11(2), 95-114. doi:10.1016/j.appsy.2004.09.001

Sameroff, A. J., \& Seifer, R. (1983). Familial risk and child competence. Child Development, 54(5), 1254-1268. doi:10.2307/1129680

Satherley, N., Milojev, P., Greaves, L. M., Huang, Y., Osborne, D., Bulbulia, J., \& Sibley, C. G. (2015). Demographic and psychological predictors of panel attrition: Evidence from the New Zealand attitudes and values study. PLoS ONE, 10(3), 1-22. doi:10.1371/journal.pone.0121950

Schaeffer, N. C., \& Presser, S. (2003). The science of asking questions. Annual Review of Sociology, 29(2003), 29, 65-88. doi:10.1146/annurev.soc.29.110702.110112

Schore, A. N. (2001a). Effects of a secure attachment relationship on right brain development, affect regulation, and infant mental health. Infant Mental Health Journal, 22(2), 7-66.

Schore, A. N. (2001b). The effects of early relational trauma on right brain development, affect regulation, and infant mental health. Infant Mental Health Journal, 22(1-2), 201269.

Selby, E. A., Franklin, J., Carson-Wong, A., \& Rizvi, S. L. (2013). Emotional cascades and self-injury: Investigating instability of rumination and negative emotion. Journal of Clinical Psychology, 69(12), 1213-1227. doi:10.1002/jclp.21966 
Selig, J. P., \& Little, T. D. (2012). Autoregressive and cross-lagged panel analysis for longitudinal data. In B. Laursen, T. D. Little, \& N. A. Card (Eds.) Handbook of Developmental Research Methods. New York, NY: The Guilford Press.

Shepard, D. S., Gurewich, D., Lwin, A. K., Reed, G. A., \& Silverman, M. M. (2016). Suicide and suicidal attempts in the United States: Costs and policy implications. Suicide and Life-Threatening Behavior, 46(3), 352-362. doi:10.1111/s1tb.12225

Smith, S. W., Daunic, A. P., Algina, J., Pitts, D. L., Merrill, K. L., Cumming, M. M., \& Allen, C. (2016). Self-regulation for students with emotional and behavioral disorders: Preliminary effects of the I Control curriculum. Journal of Emotional and Behavioral Disorders, 25(3), 143-156. doi:10.1177/1063426616661702

Stanley, B., Winchel, R., Molcho, A., Simeon, D., \& Stanley, M. (1992). Suicide and the selfharm continuum: Phenomenological and biochemical evidence. International Review of Psychiatry, 4(2), 149-155. doi:10.3109/09540269209066312

Statistics New Zealand (2013). 2013 Census QuickStats about national highlights. Available from www.stats.govt.nz

Swannell, S. V., Martin, G. E., Page, A., Hasking, P., \& St John, N. J. (2014). Prevalence of nonsuicidal self-injury in nonclinical samples: Systematic review, meta-analysis and meta-regression. Suicide and Life-Threatening Behavior, 44, 273-303. doi:10.1111/sltb.12070

Szabó, M. (2010). The short version of the Depression Anxiety Stress Scales (DASS-21): Factor structure in a young adolescent sample. Journal of Adolescence, 33(1), 1-8. doi:10.1016/j.adolescence.2009.05.014

Tatnell, R., Kelada, L., Hasking, P., \& Martin, G. (2014). Longitudinal analysis of adolescent NSSI: The role of intrapersonal and interpersonal factors. Journal of Abnormal Child Psychology, 42(6), 885-896. doi:10.1007/s10802-013-9837-6

Thompson, R. A., \& Meyer, S. (2007). Socialization of emotion regulation in the family. In J. J. Gross (Ed.), Handbook of emotion regulation, (pp. 249-268). New York, NY: Guilford Press.

Tuisku, V., Pelkonen, M., Kiviruusu, O., Karlsson, L., Ruuttu, T., \& Marttunen, M. (2009). Factors associated with deliberate self-harm behaviour among depressed adolescent outpatients. Journal of Adolescence, 32(5), 1125-1136. doi:10.1016/j.adolescence.2009.03.001

Tull, M. T., Barrett, H. M., McMillan, E. S., \& Roemer, L. (2007). A preliminary investigation of the relationship between emotion regulation difficulties and 
posttraumatic stress symptoms. Behavior Therapy, 38(3), 303-313. doi:10.1016/j.beth.2006.10.001

Vasilev, C. A., Crowell, S. E., Beauchaine, T. P., Mead, H. K., \& Gatzke-Kopp, L. M. (2009). Correspondence between physiological and self-report measures of emotion dysregulation: A longitudinal investigation of youth with and without psychopathology. Journal of Child Psychology and Psychiatry and Allied Disciplines, 50(11), 1357-1364. doi:10.1111/j.1469-7610.2009.02172.x

Velicer, W. F., Eaton, C., \& Fava, J. L. (2000). Construct explication through Factor or Component Analysis: A review and evaluation of alternative procedures for determining the number of factors or components. In R. G., Goffin \& E. Helmes (Eds.) Problems and solutions in human assessment: Honoring Douglas N. Jackson at seventy (pp. 41-71).

New York, NY: Kluwer Academic Publishers. doi:10.1007/978-1-4615-4397-8

Vispoel, W. P., Boo, J., \& Bleiler, T. (2001). Computerized and paper-and-pencil versions of the Rosenberg Self-Esteem Scale: A comparison of psychometric features and respondent preferences. Educational and Psychological Measurement, 61(3), 461-474. doi:10.1177/00131640121971329

Weinberg, A., \& Klonsky, E. D. (2009). Measurement of emotion dysregulation in adolescents. Psychological Assessment, 21(4), 616-621. doi:10.1037/a0016669

Whiteside, U., Chen, E., Neighbors, C., Hunter, D., Lo, T., \& Larimer, M. (2007).

Difficulties regulating emotions: Do binge eaters have fewer strategies to modulate and tolerate negative affect? Eating Behaviors, 8(2), 162-169.

doi:10.1016/j.eatbeh.2006.04.001

Whitlock, J. L., Eckenrode, J., \& Daniel, S. (2006). Self-injurious behaviors in a college population. Pediatrics, 117(6), 1939-1948. doi:10.1542/peds.2005-2543

Whitlock, J., Muehlenkamp, J., Purington, A., Eckenrode, J., Barreira, P., Baral Abrams, G., ... Knox, K. (2011). Nonsuicidal self-injury in a college population: General trends and sex differences. Journal of American College Health, 59(8), 691-698. doi:10.1080/07448481.2010.529626

Whitlock, J., Prussien, K., \& Pietrusza, C. (2015). Predictors of self-injury cessation and subsequent psychological growth: Results of a probability sample survey of students in eight universities and colleges. Child and Adolescent Psychiatry and Mental Health, 9:19. doi:10.1186/s13034-015-0048-5

Wilkinson, P., Kelvin, R., Roberts, C., Dubicka, B., \& Goodyer, I. (2011). Clinical and psychosocial predictors of suicide attempts and nonsuicidal self-injury in the Adolescent 
Depression Antidepressants and Psychotherapy Trial (ADAPT). American Journal of Psychiatry, 168(5), 495-501. doi:10.1176/appi.ajp.2010.10050718

Williams, F., \& Hasking, P. (2010). Emotion regulation, coping and alcohol use as moderators in the relationship between non-suicidal self-injury and psychological distress. Prevention Science, 11(1), 33-41. doi:10.1007/s11121-009-0147-8

Williams, L. E., Bargh, J. A., Nocera, C. C., \& Gray, J. R. (2009). The unconscious regulation of emotion: Nonconscious reappraisal goals modulate emotional reactivity. Emotion, 9(6), 847-854. doi:10.1037/a0017745

You, J., Leung, F., \& Fu, K. (2012). Exploring the reciprocal relations between nonsuicidal self-injury, negative emotions and relationship problems in Chinese adolescents: A longitudinal cross-lag study. Journal of Abnormal Child Psychology, 40(5), 829-836. doi:10.1007/s10802-011-9597-0

You, J., Leung, F., Fu, K., \& Lai, C. M. (2011). The prevalence of nonsuicidal self-injury and different subgroups of self-injurers in Chinese adolescents. Archives of Suicide Research, 15(1), 75-86. doi:10.1080/13811118.2011.540211

Youth Wellbeing Study (2017). Resources. Retrieved from: www.victoria.ac.nz/psyc/research/youth-and-wellbeing-study/resources

Yurkowski, K., Martin, J., Levesque, C., Bureau, J. F., Lafontaine, M. F., \& Cloutier, P. (2015). Emotion dysregulation mediates the influence of relationship difficulties on nonsuicidal self-injury behavior in young adults. Psychiatry Research, 228(3), 871-878. doi:10.1016/j.psychres.2015.05.006

Zelkowitz, R. L., \& Cole, D. A. (2016). Measures of emotion reactivity and emotion regulation: Convergent and discriminant validity. Personality and Individual Differences, 102, 123-132. doi: /10.1016/j.paid.2016.06.045 


\section{Appendices \\ Appendix A \\ Difficulties in Emotion Regulation Scale}

(DERS; Gratz \& Roemer, 2004)

The statements below relate to emotions. Please read each one and select an option that best represents how much each one applies to you.

Rating scale:

$1=$ Almost never (0-10\%)

$2=$ Sometime $(11-35 \%)$

$3=$ About half the time $(36-65 \%)$

$4=$ Most of the time $(66-90 \%)$

$5=$ Almost always $(91-100 \%)$

1. I am clear about my feelings (R)

2. I pay attention to how I feel (R)

3. I experience my emotions as overwhelming and out of control

4. I have no idea how I am feeling

5. I have difficulty making sense out of my feelings

6. I am attentive to my feelings (R)

7. I know exactly how I am feeling (R)

8. I care about what I am feeling (R)

9. I am confused about how I feel

10. When I'm upset, I acknowledge my emotions (R)

11. When I'm upset, I become angry with myself for feeling that way

12. When I'm upset, I become embarrassed for feeling that way

13. When I'm upset, I have difficulty getting work done

14. When I'm upset, I become out of control

15. When I'm upset, I believe that I will remain that way for a long time

16. When I'm upset, I believe that I'll end up feeling very depressed

17. When I'm upset, I believe that my feelings are valid and important (R)

18. When I'm upset, I have difficulty focusing on other things

19. When I'm upset, I feel out of control 
20. When I'm upset, I can still get things done (R)

21. When I'm upset, I feel ashamed with myself for feeling that way

22. When I'm upset, I know that I can find a way to eventually feel better (R)

23. When I'm upset, I feel like I am weak

24. When I'm upset, I feel like I can remain in control of my behaviours (R)

25. When I'm upset, I feel guilty for feeling that way

26. When I'm upset, I have difficulty concentrating

27. When I'm upset, I have difficulty controlling my behaviours

28. When I'm upset, I believe that there is nothing I can do to make myself feel better

29. When I'm upset, I become irritated with myself for feeling that way

30. When I'm upset, I start to feel very bad about myself

31. When I'm upset, I believe that wallowing in it is all I can do

32. When I'm upset, I lose control over my behaviours

33. When I'm upset, I have difficulty thinking about anything else

34. When I'm upset, I take time to figure out what I'm really feeling (R)

35. When I'm upset, it takes me a long time to feel better

36. When I'm upset, my emotions feel overwhelming

Non-Acceptance Subscale consists of items 11, 12, 21, 23, 25, and 29.

Goals Subscale consists of items 13, 18, 20, 26, and 33.

Impulse Subscale consists of items 3, 14, 19, 24, 27, and 32.

Aware Subscale consists of items 2, 6, 8, 10, 17, and 34 .

Strategies Subscale consists of items 15, 16, 22, 28, 30, 31, 35, and 36.

Clarity Subscale consists of items 1, 4, 5, 7, and 9. 


\section{Appendix B}

Emotion Regulation Index for Children and Adolescents

(ERICA; MacDermott, Gullone, Allen, King, \& Tonge, 2010)

Below are a number of statements. Please read each and rate how strongly you agree (or disagree) with them.

\section{Rating Scale:}

$1=$ Strongly disagree

$2=$ Disagree

$3=$ Half and half

$4=$ Agree

$5=$ Strongly agree

1. I am a happy person

2. When adults are friendly to me, I am friendly to them

3. I handle it well when things change or I have to try something new

4. When I get upset, I can get over it quickly

5. When things don't go my way I get upset easily (R)

6. When other kids are friendly to me, I am friendly to them

7. I have angry outbursts (R)

8. I enjoy seeing others hurt or upset (R)

9. I can be disruptive at the wrong times $(\mathrm{R})$

10. I get angry when adults tell me what I can and cannot do (R)

11. I am a sad person (R)

12. I have trouble waiting for something I want (R)

13. I am quiet and shy, and I don't show my feelings (R)

14. I do things without thinking about them first (R)

15. When others are upset, I become sad or concerned for them

16. I annoy others by not minding my own business (R)

Emotional Control subscale consists of items: 5, 7, 9, 10, 12, 14, and 16. Emotional Self-Awareness subscale consists of items: 1, 3, 4, 11, and 13. Situational Responsiveness subscale consists of items: 2, 6, 8, and 15. 
Appendix C

\section{Lifetime NSSI Question}

Sometimes people have thoughts about hurting themselves on purpose, but do not actually hurt themselves. And sometimes people hurt themselves deliberately (i.e., on purpose) to cause damage to their body but NOT to kill themselves (e.g. cut, burn, scratch, or carve their skin, bang or hit themselves, or prevent wounds from healing).

Please indicate whether you have had thoughts about hurting yourself on purpose (but not actually done this), whether you have hurt yourself on purpose (e.g. cut, burnt, scratched or carved your skin, etc.), or whether you have never done this:

\section{Response Scale}

$0=$ No, I have never hurt myself

$1=$ Yes, I have hurt myself on purpose

$2=\mathrm{I}$ have thought about hurting myself on purpose 
Appendix D

Deliberate Self-Harm Inventory

(DSHI-s; Lundh et al., 2007)

Have you EVER deliberately (but without wanting to kill yourself) ...

\section{Rating Scale:}

$0=$ I've never thought about doing this

$1=$ I've thought about doing this, but have never done it

$2=$ I've done this once

$3=$ I've done this a few times

$4=$ I've done this $\underline{\text { many times }}$

1. ... cut your wrist, arms, or other areas of your body?

2. ... burned yourself with a cigarette, lighter or match?

3. ... carved words, pictures, designs or other marks into your skin (not including tattoos or tā moko)?

4. ... severely scratched yourself, to the extent that scarring or bleeding occurred?

5 . ... bitten yourself, to the extent that you broke the skin?

6. ... rubbed sandpaper on your body?

7. ... dripped acid onto your skin?

8. ... used bleach, or oven cleaner to scrub your skin?

9. ... stuck sharp objects such as needles, pins, staples, etc. into your skin (not including tattoos or tā moko, ear or body piercing, or needles for drug use)?

$10 . .$. rubbed glass into your skin?

11. ... broken your own bones?

12. ... punched yourself, or banged your head against something, to the extent that caused a bruise to appear?

13. ... prevented wounds from healing?

Note. Only participants who indicated they have thought about or have hurt themselves on purpose completed this measure. Items 3 and 9 were modified to explicitly exclude tā moko, the body and face marking that is part of Māori culture. 
Appendix E

Inventory of Statements of Self-Injury

(ISAS; Klonsky \& Glenn, 2009)

People usually describe a range of reasons for deliberately hurting themselves. Some of these are described below. Please read each one, and tick the box that seems most true for you.

\section{Rating Scale:}

$0=$ Not relevant

$1=$ Somewhat relevant

$2=$ Very relevant

When I self-injure, I am ...

1. ... calming myself down

2. ... creating a boundary between myself and others

3. ... punishing myself

4. ... giving myself a way to care for myself (by attending to the wound)

5. ... causing pain so I will stop feeling numb

6. ... avoiding the urge to attempt suicide

7. ... doing something to feel excited (get a buzz)

8. ... bonding with peers (to be close to other people)

9. ... letting others know the extent of my emotional pain

$10 . .$. seeing if I can stand the pain

11 .... creating a physical sign that I feel awful

12. ... getting back at someone

13. ... ensuring that I am self-sufficient (that I can do things for myself)

14. ... releasing emotional pressure that has built up inside of me

15. ... showing that I am separate from other people

16. ... expressing anger towards myself for being worthless or stupid

17. ... creating a physical injury that is easier to care for than my emotional distress

18. ... trying to feel something (as opposed to nothing) even if it is physical pain

19. ... responding to suicidal thoughts without actually attempting suicide

20 .... entertaining myself or others by doing something extreme

21.... fitting in with others 
22. ... seeking care or help from others

23.... showing I am tough or strong

24. ... proving to myself that my emotional pain is real

25 .... getting revenge against others

26. ... showing that I do not need to rely on others for help

27. ... reducing anxiety, frustration, anger, or other overwhelming emotions

28. ... establishing a barrier between myself and others

29 . ... reacting to feeling unhappy with myself or disgusted with myself

30 .... allowing myself to focus on treating the injury, which can feel satisfying

31. ... making sure I am still alive when I don't feel real

32. ... putting a stop to suicidal thoughts

33. ... pushing my limits (like skydiving or other extreme activities)

34. ... creating a sign of friendship or kinship with friends or loved ones

35. ... keeping a loved one from leaving or abandoning me

36. ... proving I can take the physical pain

37. ... showing the emotional distress I'm experiencing

38. ... trying to hurt someone close to me

39 .... establishing that I am autonomous/independent

Note. Only participants who indicated that they had engaged in NSSI complete this measure.

Affect Regulation is consist of items 1, 14, and 27.

Interpersonal Boundaries consists of items 2, 15, and 28.

Self-Punishment consists of item 3, 16, and 29.

Self-Care consists of items 4, 17, and 30.

Anti-Dissociation/Feeling Generation consist of items 5, 18, and 31.

Anti-Suicide consists of items 6, 19, and 32.

Sensation-Seeking consists of items 7, 20, and 33.

Peer-Bonding consist of items 8, 21, and 34.

Interpersonal Influence consists of items 9, 22, and 35.

Toughness consists of items 10, 23, and 36.

Marking Distress consists of items 11, 24, and 37.

Revenge consists of items 12, 25, and 38 .

Autonomy consists of items 13, 26, and 39. 


\author{
Appendix F \\ Depression Anxiety Stress Scale
}

(DASS-21; Henry \& Crawford, 2005)

Please read each statement and indicate how much the statement applied to you over the last week. There are no right or wrong answers. Do not spend too much time on any statement.

\title{
Rating Scale:
}

$0=$ Did not apply to me at all

$1=$ Applied to me to some degree, or some of the time

2 = Applied to me to a considerable degree, or a good part of time;

$3=$ Applied to me very much, or most of the time

1. I found it hard to wind down

2. I was aware of dryness of my mouth

3. I couldn't seem to experience any positive feeling at all

4. I experienced breathing difficulty (eg, excessively rapid breathing, breathlessness in the absence of physical exertion)

5. I found it difficult to work up the initiative to do things

6. I tended to over-react to situations

7. I experienced trembling (eg, in the hands)

8. I felt that I was using a lot of nervous energy

9. I was worried about situations in which I might panic and make a fool of myself

10. I felt that I had nothing to look forward to

11. I found myself getting agitated

12. I found it difficult to relax

13. I felt down-hearted and blue

14. I was intolerant of anything that kept me from getting on with what I was doing

15. I felt I was close to panic

16. I was unable to become enthusiastic about anything

17. I felt I wasn't worth much as a person

18. I felt that I was rather touchy

19. I was aware of the action of my heart in the absence of physical exertion (eg, sense of heart rate increase, heart missing a beat) 
20. I felt scared without any good reason

21. I felt that life was meaningless

Depression Subscale consists of items 3, 5, 10, 13, 16, 17, and 21.

Anxiety Subscale consists of items 2, 4, 7, 9, 15, 19, and 20.

Stress Subscale consists of items 1, 6, 8, 11, 12, 14, and 18.

Note. In order to prevent misunderstanding, the original item 'I felt down-hearted and blue' was modified to 'I felt down-hearted and sad'. The Stress subscale was not included in analyses within this thesis. 
Appendix G

Rosenberg Self-Esteem Scale

(RSES; Rosenberg, 1965)

Below is a list of statements dealing with your general feelings about yourself. Please read them and rate how strongly you agree (or disagree) with them.

Rating Scale:

$1=$ Strongly disagree

$2=$ Disagree

$3=$ Agree

$4=$ Strongly Agree

1. Overall, I am satisfied with myself

2. At times, I think I am no good at all (R)

3. I feel that I have a number of good qualities

4. I am able to do things as well as most people

5. I feel I do not have much to be proud of $(\mathrm{R})$

6. I certainly feel useless at times (R)

7. I feel that I'm a person of worth, worth at least as much as other people

8. I wish I could have more respect for myself (R)

9. Generally, I tend to feel that I am a failure (R)

10. I take a positive attitude to myself 


\section{Appendix $\mathrm{H}$}

Study One NSSI Lifetime Prevalence and Functions

Table A

Endorsement of different NSSI behaviours among participants who reported engaging in NSSI in Study One.

\begin{tabular}{ll}
\hline NSSI Behaviour & Percentage of NSSI subsample \\
\hline Cutting skin & $64.1 \%$ \\
Scratching skin & $55.6 \%$ \\
Punched or banged self & $39.2 \%$ \\
Carving images or words on skin & $29.4 \%$ \\
Sticking sharp objects into skin & $28.8 \%$ \\
Prevented wounds from healing & $27.5 \%$ \\
Burning skin & $19.0 \%$ \\
Bitten self & $18.3 \%$ \\
Rubbed glass into skin & $7.2 \%$ \\
Rubbed sandpaper on skin & $3.9 \%$ \\
Broken bones & $1.3 \%$ \\
Bleach on skin & $0.7 \%$ \\
Dripped acid on skin & $0.0 \%$ \\
\hline
\end{tabular}

$N=153$. 
Table B

Functions of NSSI among participants who reported engaging in NSSI in Study One.

\begin{tabular}{|c|c|c|}
\hline \multirow[t]{2}{*}{ Function } & \multicolumn{2}{|c|}{ NSSI subsample } \\
\hline & Percentage Endorsement & $M$ endorsement $(S D)$ \\
\hline Affect Regulation & $90.4 \%$ & $1.14(0.64)$ \\
\hline Self-Punishment & $84.9 \%$ & $0.99(0.67)$ \\
\hline Anti-Dissociation & $70.6 \%$ & $0.62(0.57)$ \\
\hline Anti-Suicide & $49.2 \%$ & $0.48(0.61)$ \\
\hline Marking Distress & $48.4 \%$ & $0.34(0.47)$ \\
\hline Toughness & $45.2 \%$ & $0.25(0.37)$ \\
\hline Self-Care & $41.3 \%$ & $0.26(0.40)$ \\
\hline Interpersonal Boundaries & $40.4 \%$ & $0.28(0.43)$ \\
\hline Sensation Seeking & $27.8 \%$ & $0.14(0.27)$ \\
\hline Interpersonal Influence & $27.0 \%$ & $0.14(0.29)$ \\
\hline Autonomy & $16.7 \%$ & $0.10(0.28)$ \\
\hline Revenge & $10.3 \%$ & $0.06(0.21)$ \\
\hline Peer Bonding & $6.3 \%$ & $0.03(0.15)$ \\
\hline
\end{tabular}

$N=126$. 


\section{Appendix I}

Inventory of Parent and Peer Attachment

(IPPA; Armsden \& Greenberg, 1987)

The following statement ask about your feelings about your friends, and your parents or the people who act as parents for you. If you have more than one person acting as either your mother or father (e.g., step-parent of member of your whānau who looks after you like a parent), please answer the questions for the one you feel has most influenced you,

Please read each statement and rate how true (or untrue) each is for you.

\section{Rating Scale:}

$1=$ Almost never or never true

$2=$ Seldom true

$3=$ Sometimes true

$4=$ Often true

$5=$ Almost always or always true

1. My parents respect my feelings

2. My parents accept me as I am

3. I get upset more than my parents know about (R)

4. When we discuss things, my parents consider my point for view

5. My parents trust my judgement

6. I tell my parents about my problems and troubles

7. My parents encourage me to talk about my difficulties

8. I don't know whom I can depend on these days (R)

9. My parents don't understand what I'm going through these days (R)

10. I can count on my parents when I need to get something off my chest

11. I feel that no one understands me $(\mathrm{R})$

12. If my parents know something is bothering me, they ask about it

13. My friends sense when I'm upset about something

14. Talking over my problems with my friends makes me feel ashamed or foolish (R)

15. My friends encourage me to talk about my difficulties

16. My friends don't understand what I'm going through these days (R) 
17. My friends listen to what I have to say

18. I feel my friends are good friends

19. I trust my friends

20. My friends respect my feelings

21. I get upset more than my friends know about (R)

22. It seems as if my friends are irritated with me for no reason $(\mathrm{R})$

23. I tell my friends about my problems and troubles

24. If my friends know something is bothering me, they ask me about it

Parental Attachment Subscale consists of items 1 through 12.

Peer Attachment Subscale consists of items 13 through 24. 
Appendix $\mathbf{J}$

Study Two Lifetime NSSI Prevalence and Functions

Table C

Lifetime endorsement of different NSSI behaviours among participants who reported engaging in NSSI across three waves in Study Two and Three.

\begin{tabular}{lccc}
\hline NSSI Behaviour & \multicolumn{3}{c}{ Percentage of NSSI subsample } \\
\cline { 2 - 4 } & T1 & T2 & T3 \\
\hline Cutting & $55.0 \%$ & $54.8 \%$ & $60.0 \%$ \\
Scratching & $41.4 \%$ & $45.2 \%$ & $55.2 \%$ \\
Carving images or words & $30.0 \%$ & $27.4 \%$ & $28.8 \%$ \\
Punched or banged self & $28.6 \%$ & $33.3 \%$ & $40.8 \%$ \\
Prevented wounds from healing & $22.9 \%$ & $30.4 \%$ & $32.3 \%$ \\
Sharp objects into skin & $21.2 \%$ & $26.6 \%$ & $32.0 \%$ \\
Bitten self & $12.4 \%$ & $15.1 \%$ & $17.6 \%$ \\
Burning & $10.3 \%$ & $15.3 \%$ & $16.3 \%$ \\
Rubbed glass into skin & $6.1 \%$ & $7.1 \%$ & $6.4 \%$ \\
Broken bones & $4.1 \%$ & $0.8 \%$ & $3.2 \%$ \\
Rubbed sandpaper on skin & $2.1 \%$ & $4.8 \%$ & $2.4 \%$ \\
Dripped acid on skin & $0.0 \%$ & $2.4 \%$ & $1.6 \%$ \\
Bleach & $0.0 \%$ & $0.8 \%$ & \\
\hline
\end{tabular}

T1 $N=102, \mathrm{~T} 2 N=126, \mathrm{~T} 3 N=125$ 
Table D

Lifetime endorsement functions of NSSI among participants who reported engaging in NSSI across three waves in Study Two and Three.

\begin{tabular}{|c|c|c|c|}
\hline \multirow[t]{2}{*}{ Function } & \multicolumn{3}{|c|}{ Percentage of NSSI subsample } \\
\hline & $\mathrm{T} 1$ & $\mathrm{~T} 2$ & $\mathrm{~T} 3$ \\
\hline Affect Regulation & $90.5 \%$ & $91.0 \%$ & $93.9 \%$ \\
\hline Self-Punishment & $75.3 \%$ & $82.2 \%$ & $85.4 \%$ \\
\hline Marking Distress & $55.9 \%$ & $53.0 \%$ & $54.9 \%$ \\
\hline Anti-Dissociation & $54.3 \%$ & $45.0 \%$ & $62.2 \%$ \\
\hline Anti-Suicide & $38.7 \%$ & $47.0 \%$ & $48.8 \%$ \\
\hline Toughness & $37.6 \%$ & $48.0 \%$ & $46.3 \%$ \\
\hline Interpersonal Boundaries & $36.6 \%$ & $42.0 \%$ & $41.5 \%$ \\
\hline Self-Care & $31.9 \%$ & $20.0 \%$ & $40.2 \%$ \\
\hline Interpersonal Influence & $25.5 \%$ & $29.0 \%$ & $29.3 \%$ \\
\hline Autonomy & $22.3 \%$ & $27.0 \%$ & $30.5 \%$ \\
\hline Sensation Seeking & $21.5 \%$ & $27.0 \%$ & $25.6 \%$ \\
\hline Peer Bonding & $17.2 \%$ & $12.0 \%$ & $9.8 \%$ \\
\hline Revenge & $14.7 \%$ & $9.0 \%$ & $8.5 \%$ \\
\hline
\end{tabular}

T1 $N=102, \mathrm{~T} 2 N=126, \mathrm{~T} 3 N=125$ 